\title{
Variedades Satélites
}

\author{
Rui Marcos de O. Barros
}

ORIENTADOR: OzIRIDE MANZOLI NETO

Tese apresentada ao Instituto de Ciências Matemáticas de São Carlos - USP, como parte dos requisitos para obtenção do Título de Doutor em Ciências - Área: Matemática - Geometria e Topologia.

São Carlos

Dezembro-1994 


\section{Abstract}

Let $V_{F} \cong_{\phi} F^{k} \times D^{2}$ be a trivialization by $\phi$ of a tubular neighborhood of an embedding of an orientable manifold $F^{k}$ in $S^{k+2}$. In this work we define embeddings of certain manifolds $M^{k}$ in $V_{F}$. These manifolds are defined in such way that the map $\pi: V_{F} \cong{ }_{\phi} F^{k} \times D^{2} \rightarrow F^{k}$ restricted to $M^{k}$ is an $n$-covering map of $F^{k}$. We call these manifolds satellites of $F^{k}$ since it is a generalization of a satellite construction in the classical case. We prove that there exists a Seifert Manifold $W^{k+1}$ for $M^{k}$ built of $n$-parallel copies of a Seifert Manifold for $F^{k}$ outside $V_{F}$. Using $W^{k+1}$ it is possible to relate many invariants of the embedding of $F^{k}$ with those of the embedding of the manifold $M^{k}$. In particular we study the relation between the Alexander Modules of the two embeddings using a special decomposition of the Abelian Covering $\tilde{X}_{M}$ of $S^{k+2}-V_{M}\left(V_{M} \cong M \times D^{2}\right)$. For the case of orientable surfaces in $S^{4}$ we are able to get more informations and examples. 


\section{Variedades Satélites RUI MARCOS DE O. BARROS \\ ORIENTADOR: OZIRIDE MANZOLI NETO}

\section{RESUMO}

Seja $f: F^{k} \rightarrow S^{k+2}$ um mergulho de uma variedade orientada fechada $F^{k}$ em uma esfera $(k+2)$-dimensional $S^{k+2}$. Denotemos por $V$ uma vizinhança tubular aberta de $f\left(F^{k}\right)$. Neste trabalho construimos em $V$ uma variedade $k$-dimensional $M$ que é o espaço total de um recobrimento de $n$ folhas de $F^{k}$. Esta construção generaliza a construção de satélites de nós clássicos, por isso chamamos $M$ de variedade satélite de $F_{k}$. Usando a variedade de Seifert $W$ do mergulho $f\left(F^{k}\right)$, mostramos que existe variedade de Seifert $S$ para o mergulho satélite $g: M \rightarrow V \subset S^{k+2}$ tal que em $S^{k+2}-V$ a variedade $S$ é formada por $n$ cópias paralelas de $W$. Utilizando a variedade $S$ conseguimos fazer uma decomposição no espaço total $\tilde{X}_{m}$ do recobrimento cíclico infinito do complementar $X_{m}=S^{k+2}-g(M)$. Esta decomposição possibilita comparar os módulos de Alexander $H_{*}\left(\tilde{X}_{M}\right)$ do satélite, com os módulos $H_{*}\left(\tilde{X}_{F}\right)$ do mergulho inicial. Apresentamos no início do trabalho uma caracterização algébrica dos módulos de Alexander para mergulhos de superfícies orientadas fechadas $F^{2}$ em uma esfera $S^{4}$. Utilizando essa caracterização calculamos os módulos de Alexander de alguns exemplos de construção de satélites bidimensionais. 
Este trabalho dependeu parcialmente do financiamento da CAPES através de seu programa PICD.

Agradecimentos especiais pelas sugestões aos professores Derek Hacon (PUC-Rio) e Osamu Saeki (Hiroshima Univ.) . 


\title{
Variedades Satélites
}

\author{
Rui Marcos de Oliveira Barros
}

\section{Introdução}

Os trabalhos de Levine J. [18] e de Neto O. M. [26] caracterizam os módulos de Alexander de mergulhos $S^{k} \rightarrow S^{k+2}$ e os "Total Linking Number Modules" para links $L^{k} \rightarrow S^{k+2}$. Na primeira parte deste trabalho são apresentados teoremas importantes sobre os Módulos de Alexander de mergulhos de superfícies orientadas de genus " $g$ " na esfera $S^{4}$, o que é um primeiro passo de generalização dos trabalhos citados. Esses teoremas serão utilizados para o cálculo dos módulos de Alexander de superfícies satélites na segunda parte.

Na segunda parte ampliamos a noção de "nó satélite" introduzida por Seifert H. [30] e estendida por Shinohara Y. [33], para mergulhos de variedades $n$-dimensionais nas esferas $(n+2)$-dimensionais. Pois a análise do caso de mergulhos de superfícies $F_{g}^{2}$ em $S^{4}$ nos propiciou o estudo em dimensões maiores.

Aqui estaremos sempre trabalhando na categoria $C^{\infty}$.

Observação: Utilizaremos neste trabalho duas sequências espectrais associadas ao recobrimento $p: \tilde{X} \rightarrow X$ cíclico infinito. Devido ao tempo excessivo que gastaríamos para introduzir tal assunto vamos apenas apresentá-las e utilizá-las como instrumento de cálculo para chegar aos nossos objetivos. Excelente texto introdutório nesse assunto é [28] e para um estudo bem mais completo sugerimos [21]. Pode-se encontrar também esse assunto em outros textos como por exemplo [35] e $[8]$.

Considere o recobrimento $p: \tilde{X} \rightarrow X$ com grupo de transformações $G \cong \mathbb{Z}$. Então existem as seguintes sequências espectrais:

Sequência Espectral de Cartan-Leray

Consiste de uma coleção indexada em $i \in \mathbb{N}, i \geq 2$, de módulos (sobre o anel 
de grupo $\left.\Lambda=\mathbb{Z}\left[t, t^{-1}\right]\right)$ bigraduados $E_{p, q}^{i}$, e uma coleção de morfismos de bigrau $(-r, r-1)$ como segue.

$$
d^{r}: E_{p, q}^{r} \longrightarrow E_{p-r, q+r-1}^{r}
$$

Valem os isomorfismos

$$
\begin{gathered}
E_{p, q}^{r+1}=H\left(E_{p, q}^{r}, d^{r}\right)=\frac{\text { Ker } d_{1}^{r}}{\operatorname{Im} d_{2}^{r}}, \text { onde } \\
E_{p+r, q-r+1}^{r} \stackrel{d_{2}^{r}}{\longrightarrow} E_{p, q}^{r} \stackrel{d_{1}^{r}}{\longrightarrow} E_{p-r, q+r-1}^{r} .
\end{gathered}
$$

A sequência $\left(E_{p, q}^{i}\right)_{i \geq 2}$ tem um limite denotado por $E_{p, q}^{\infty}$, isto é, existe $n \in$ IN tal que $E_{p, q}^{i} \cong E_{p, q}^{\infty}, \quad i \geq n$.

A sequência de Cartan-Leray converge para $H_{*}(X)$, isto é:

Existe uma filtração

$$
H_{p+q}(X)=L_{i, 0} \supset L_{i-1,1} \supset \ldots \supset L_{0, i} \supset L_{-1, i+1}=0, i=p+q
$$

tal que

$$
\frac{L_{s, t}}{L_{s-1, t+1}} \cong E_{s, t}^{\infty}
$$

E finalmente vale o isomorfismo:

$$
E_{p, q}^{2}=H_{p}\left(\mathbb{Z} ; H_{q}(\tilde{X})\right)
$$

Sequência Espectral dos Coeficientes Universais

Consiste de uma coleção indexada em $i \in \mathbb{N}, \quad i \geq 2$, de módulos bigraduados $E_{i}^{p, q}$ e uma coleção de morfismos $d_{r}$ de bigrau $(1-r, r)$ como segue.

$$
d_{r}: E_{r}^{p, q} \longrightarrow E_{r}^{p+1-r, q+r}
$$

Valem os isomorfismos 


$$
\begin{gathered}
E_{r+1}^{p, q}=H\left(E_{r}^{p, q}, d_{r}\right)=\frac{\text { Ker } d_{r}^{1}}{\operatorname{Im} d_{r}^{2}}, \text { onde } \\
E_{r}^{p-1+r, q-r} \stackrel{d_{r}^{2}}{\longrightarrow} E_{r}^{p, q} \stackrel{d_{r}^{1}}{\longrightarrow} E_{r}^{p+1-r, q+r} .
\end{gathered}
$$

A sequência $\left(E_{i}^{p, q}\right)_{i \geq 2}$ tem um limite denotado por $E_{\infty}^{p, q}$, isto é, existe $n \in$ IN tal que $E_{i}^{p, q} \cong E_{\infty}^{p, q}, i \geq n$.

Esta sequência espectral converge para a cohomologia equivariante $H_{e}^{*}(\tilde{X})$, isto é, existe uma filtração

$$
H_{e}^{p+q}(\tilde{X})=J_{i, 0} \supset J_{i-1,1} \supset \ldots \supset J_{0, i} \supset J_{-1, i+1}=0, i=p+q
$$

tal que

$$
\frac{J_{r, s}}{J_{r-1, s+1}} \cong E_{\infty}^{r, s} .
$$

E finalmente vale o isomorfismo

$$
E_{2}^{p, q}=\operatorname{Ext}_{\Lambda}^{q}\left[H_{p}(\tilde{X}), \Lambda\right] .
$$

A definição de cohomologia equivariante é

$$
H_{e}^{*}(\tilde{X})=H^{*}\left[H o m_{\Lambda}(C(\tilde{X}), \mathbb{Z})\right] .
$$

OBS: Sempre que não escrevermos o anel de coeficientes de um grupo de homologia ou cohomologia estaremos considerando que tal coeficiente é o anel dos inteiros Th. 


\section{Módulos de Alexander}

Denotamos por:

$F_{g}^{2}$ uma superfície orientável de genus " $g$ ",

$L$ a imagem de um mergulho $f: F_{g}^{2} \rightarrow S^{4}$,

$U$ uma vizinhança tubular aberta de $L$ em $S^{4}$,

$X(L)=S^{4}-U($ ou simplesmente $X)$.

Podemos calcular homologia e cohomologia de $X$ usando sequências exatas e dualidades. Esses grupos não dependem da particular classe de $L$, dependem apenas do genus de $F_{g}^{2}$.

\section{Lema 1}

$$
H_{i}(X) \cong\left\{\begin{array} { l l } 
{ \mathbb { Z } } & { i = 0 , i = 1 } \\
{ \mathbb { Z } ^ { 2 g } } & { i = \mathscr { 2 } } \\
{ 0 } & { \text { outros } }
\end{array} \quad H _ { i } ( X , \partial X ) \cong \left\{\begin{array}{ll}
\mathbb{Z} & i=3, i=4 \\
\mathbb{Z}^{2 g} & i=\mathscr{Q} \\
0 & \text { outros }
\end{array}\right.\right.
$$

Dem. Temos:

$$
H_{4-i}\left(S^{4}-U\right) \cong H^{i}\left(S^{4}, S^{4}-\left(S^{4}-U\right)\right) \cong H^{i}\left(S^{4}, U\right) \cong H^{i}\left(S^{4}, L\right) .
$$

Este último isomorfismo é devido a existência de retração por deformação (forte) $r: U \rightarrow L$.

Analisando a sequência longa de cohomologia do par $\left(S^{4}, L\right)$ obtemos:

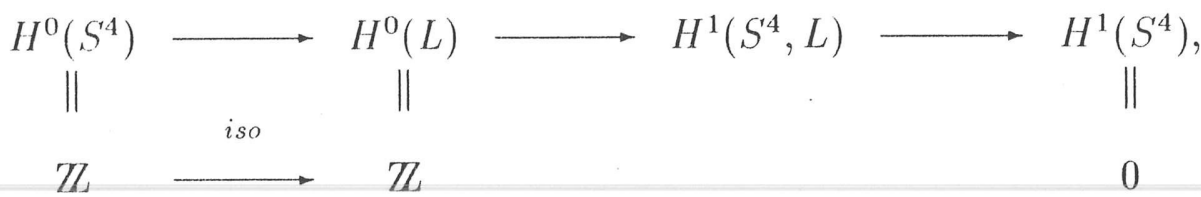

que implica $0 \cong H^{1}\left(S^{4}, L\right) \cong H_{3}(X)$,

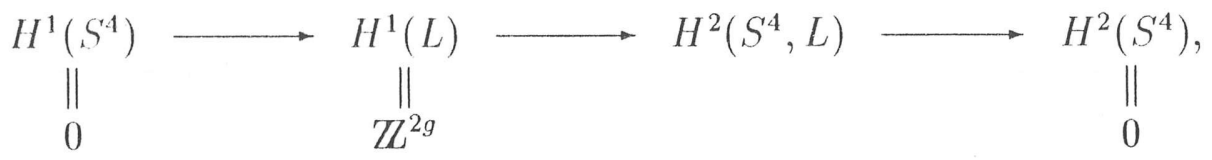


que implica $\mathbb{Z}^{2 g} \cong H^{2}\left(S^{4}, L\right) \cong H_{2}(X)$,

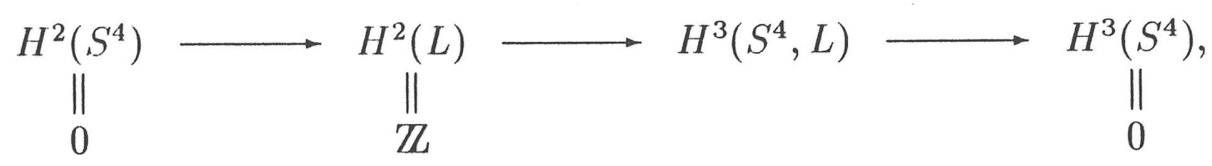

que implica $\mathbb{Z} \cong H^{3}\left(S^{4}, L\right) \cong H_{1}(X)$, e

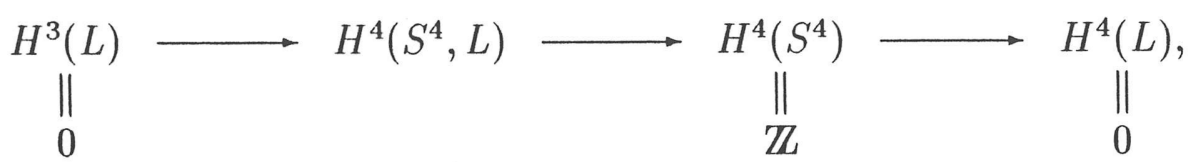

que implica $\mathbb{Z} \cong H^{4}\left(S^{4}, L\right) \cong H_{0}(X)$.

$X$ é uma variedade de dimensão 4 com bordo logo:

$$
\begin{aligned}
& H_{i}(X) \cong 0, i \geq 4, \\
& H_{0}(X, \partial X) \cong 0, \\
& H_{1}(X, \partial X) \cong H^{3}(X) \cong \operatorname{Ext}_{\mathbb{Z}}\left[H_{2}(X), \mathbb{Z}\right] \oplus \operatorname{Hom}\left(H_{3}(X), \mathbb{Z}\right) \cong 0, \\
& H_{2}(X, \partial X) \cong H^{2}(X) \cong \operatorname{EXT}_{\mathbb{Z}}\left[H_{1}(X), \mathbb{Z}\right] \oplus \operatorname{Hom}\left(H_{2}, \mathbb{Z}\right) \cong \mathbb{Z}^{2 g}, \\
& H_{3}(X, \partial X) \cong H^{1}(X) \cong \operatorname{Hom}\left(H_{1}(X), \mathbb{Z}\right) \oplus \operatorname{Ext} t_{\mathbb{Z}}\left(H_{0}(X), \mathbb{Z}\right) \cong \mathbb{Z}, \\
& H_{4}(X, \partial X) \cong H^{0}(X) \cong \operatorname{Hom}\left(H_{0}(X), \mathbb{Z}\right) \cong \mathbb{Z}, \\
& H_{i}(X, \partial X) \cong H^{4-i}(X) \cong 0, i \geq 5 .
\end{aligned}
$$

Usando o teorema de existência e unicidade de recobrimentos, a abelianização

$$
A: \pi_{1}(X) \longrightarrow \frac{\pi_{1}(X)}{\left[\pi_{1}(X), \pi_{1}(X)\right]}=H_{1}(X) \cong \mathbb{Z}
$$

dá origem a um recobrimento chamado recobrimento cíclico infinito denotado por $\tilde{X} \rightarrow X$ com grupo de transformação $\mathbb{Z}$ que torna $H^{i}(\tilde{X})$ e $H_{i}(\tilde{X})$ módulos sobre o anel de grupo $\Lambda=\mathbb{Z}[\mathbb{Z}]=\mathbb{Z}\left[t, t^{-1}\right]$.

Podemos também fazer uma construção geométrica deste recobrimento: 
Observando que o fibrado normal de $L$ em $S^{4}$ é trivial (demonstração mais adiante) é possível tomar uma trivialização da vizinhança tubular $\bar{U}$ de $L$ $\phi: \bar{U} \cong F_{g}^{2} \times D^{2}$ tal que a composta $\pi_{2} \circ \theta: \partial X \rightarrow S^{1}$ (onde $\pi_{2}$ denota a projeção no segundo fator e $\theta=\phi \mid \partial \bar{U}$ é o homeomorfismo $\left.\partial X \rightarrow F_{g}^{2} \times S^{1}\right)$ se estende a todo o espaço $X$. Essa extensão permite-nos encontrar uma variedade $V^{3}$ de dimensão 3 em $S^{4}$ cujo bordo $\partial V^{3}$ contido em $F_{g}^{2} \times S^{1}=\partial X$ é isotópico a $L$. $V^{3}$ é obtida como a imagem inversa de um valor regular em $S^{1}$.

Essa variedade $V^{3} \subset X$ é chamada Variedade de Seifert para $L$.

O recobrimento $\tilde{X}$ pode ser reconstruido com o auxílio desta variedade que é sempre bicolar; Cortamos $X$ ao longo de $V$ e obtemos um espaço $Y$. "Colando" convenientemente " $\mathbb{Z}$ " cópias de $Y$ ao longo dos cortes indexados obtemos um espaço que chamamos $\bar{X}$.

Tomamos a projeção natural que provém desta construção $p: \bar{X} \rightarrow X$ e ao observarmos que temos como grupo de transformação de recobrimento o grupo $\mathbb{Z}$ podemos através do Teorema de Existência e Unicidade afirmar que o recobrimento assim costruido é isomorfo ao cíclico infinito $\tilde{X} \rightarrow X$.

Para maiores detalhes dessa construção citamos como referência o texto de Rolfsen [29].

Podemos ver pela construção que o bordo de $\tilde{X}$ é homeomorfo ao produto cartesiano $F_{g}^{2} \times \mathbb{R}$.

OBS: Ao escrevermos os grupos abelianos $H_{i}(X), H_{i}(X, \partial X)$ ou $H_{i}(\partial X)$, estaremos considerando-os também como $\Lambda$-módulos triviais.

Lema 2 As sequências espectrais de Cartan-Leray associadas aos recobrimentos $p: \tilde{X} \rightarrow X, p:(\tilde{X}, \partial \tilde{X}) \rightarrow(X, \partial X)$ e $p: \partial \tilde{X} \rightarrow \partial X$ dão origem as seguintes sequências longas exatas de $\Lambda$-módulos:

$$
\ldots \rightarrow H_{i}(\tilde{X}) \stackrel{.(t-1)}{\longrightarrow} H_{i}(\tilde{X}) \stackrel{p_{*}}{\longrightarrow} H_{i}(X) \stackrel{\partial}{\longrightarrow} H_{i-1}(\tilde{X}) \rightarrow \ldots,
$$

$$
\begin{aligned}
\ldots & \rightarrow H_{i}(\tilde{X}, \partial \tilde{X}) \stackrel{.(t-1)}{\longrightarrow} H_{i}(\tilde{X}, \partial \tilde{X}) \stackrel{p_{*}}{\longrightarrow} H_{i}(X, \partial X) \stackrel{\partial}{\longrightarrow} H_{i-1}(\tilde{X}, \partial \tilde{X}) \rightarrow \cdots, \\
& \ldots H_{i}(\partial \tilde{X}) \stackrel{.(t-1)}{\longrightarrow} H_{i}(\partial \tilde{X}) \stackrel{p_{*}}{\longrightarrow} H_{i}(\partial X) \stackrel{\partial}{\longrightarrow} H_{i-1}(\partial \tilde{X}) \rightarrow \cdots
\end{aligned}
$$


Dem. Vamos fazer a demonstração para $p: \tilde{X} \rightarrow X$. A obtenção das outras sequências é análoga.

O $\Lambda$-módulo trivial $\mathbb{Z}$ tem dimensão homológica igual a um, pois podemos tomar a seguinte resolução projetiva:

$$
0 \longrightarrow \stackrel{{ }^{(t-1)}}{\longrightarrow} \Lambda \longrightarrow \mathbb{Z} \longrightarrow 0 .
$$

Aqui o segundo morfismo é a multiplicação por " $t-1$ ".

Efetuando o procedimento padrão para o cálculo das homologias do grupo aditivo $\mathbb{Z}$ com coeficientes no $\mathbb{Z}[\mathbb{Z}]$-módulo $H_{i}(\tilde{X})$ obtemos a seguinte sequência:

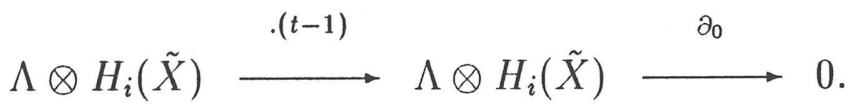

Utilizando a sequência acima vemos que:

$$
\begin{gathered}
E_{r, s}^{2}=H_{r}\left(\mathbb{Z}, H_{s}(\tilde{X})\right)=0 \text { se } r \neq 0,1, \\
E_{0, i}^{2}=H_{0}\left(\mathbb{Z}, H_{i}(\tilde{X})\right)=\operatorname{coker}\left[.(t-1): H_{i}(\tilde{X}) \rightarrow H_{i}(\tilde{X})\right], \\
E_{1, i-1}^{2}=H_{1}\left(\mathbb{Z}, H_{i-1}(\tilde{X})\right)=\operatorname{ker}\left[.(t-1): H_{i-1}(\tilde{X}) \rightarrow H_{i-1}(\tilde{X})\right] .
\end{gathered}
$$

Como para nosso caso a sequência colapsa, isto é, $E_{p, q}^{2}=E_{p, q}^{\infty}$ temos que o "edge-homomorphism" é o isomorfismo $E_{p, q}^{2} \longrightarrow E_{p, q}^{\infty}$.

Mas da filtração

$$
H_{i}(X)=L_{i, 0} \supset L_{i-1,1} \supset \ldots \supset L_{0, i} \supset 0
$$

sabemos que:

$$
L_{1, i-1}=L_{2, i-2}=L_{3, i-3}=\ldots=L_{i, 0}=H_{i}(X),
$$




$$
\begin{gathered}
L_{0, i}=E_{0, i}^{\infty}=E_{0, i}^{2}, \\
\frac{L_{1, i-1}}{L_{0, i}}=E_{1, i-1}^{\infty}=E_{1, i-1}^{2} .
\end{gathered}
$$

Ou seja,

$$
\frac{H_{i}(X)}{E_{0, i}^{2}}=E_{1, i-1}^{2}
$$

Portanto temos a sequência exata:

$$
\ldots \rightarrow H_{i}(\tilde{X}) \stackrel{.(t-1)}{\longrightarrow} H_{i}(\tilde{X}) \longrightarrow H_{i}(X) \longrightarrow H_{i-1}(\tilde{X}) \stackrel{.(t-1)}{\longrightarrow} \ldots
$$

Lema $3 \partial: H_{3}(\partial X) \rightarrow H_{2}(\partial \tilde{X})$ é um isomorfismo e ambos são isomorfos a $\mathbb{Z}$.

Dem. Observe que como $\partial \tilde{X}$ é homeomorfo a $F_{g}^{2} \times \mathbb{R}$ temos $H_{3}(\partial \tilde{X}) \cong 0$ e a aplicação $.(t-1): H_{i}(\partial \tilde{X}) \rightarrow H_{i}(\partial \tilde{X})$ é um homomorfismo nulo já que se $[\alpha] \in H_{i}(\partial \tilde{X})$ então temos um "cilindro" $\alpha \times J$, onde $J$ é um intervalo real de módulo igual a $|t|$, contido em $C_{i+1}(\partial \tilde{X})$ de "altura" $|t|$ tal que seu bordo é $\partial(\alpha \times J)=t \alpha-\alpha$.

Portanto $\mathbb{Z} \cong\left\langle\left[F_{g}^{2} \times S^{1}\right]\right\rangle=H_{3}(\partial X) \cong H_{2}(\partial \tilde{X})=\left\langle\left[F_{g}^{2}\right]\right\rangle \cong \mathbb{Z}$.

Lema $4 H_{2}(\partial X) \cong \mathbb{Z} \oplus \mathbb{Z}^{2 g}$.

Dem. Observe que $H_{1}(\partial \tilde{X}) \cong H_{1}\left(F_{g}^{2}\right) \cong \mathbb{Z}^{2 g}$ é um grupo abeliano livre (tem $2 g$ geradores) logo a sequência abaixo cinde.

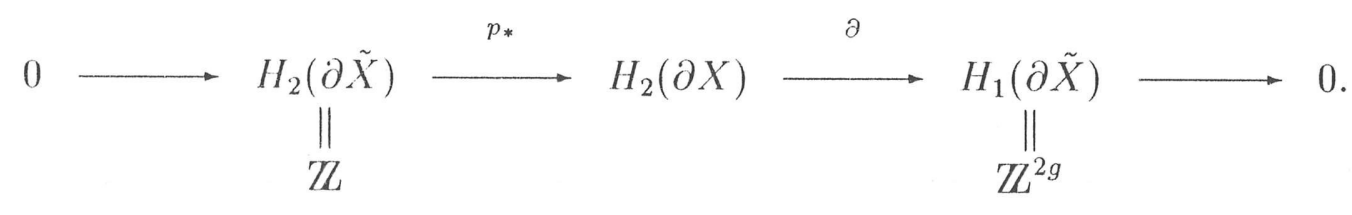


Lema $5 \quad H_{1}(\partial X) \cong \mathbb{Z}^{2 g} \oplus \mathbb{Z}$.

Dem. Observe que $H_{0}(\partial \tilde{X}) \cong \mathbb{Z}$ é um grupo abeliano livre logo a sequência abaixo cinde.

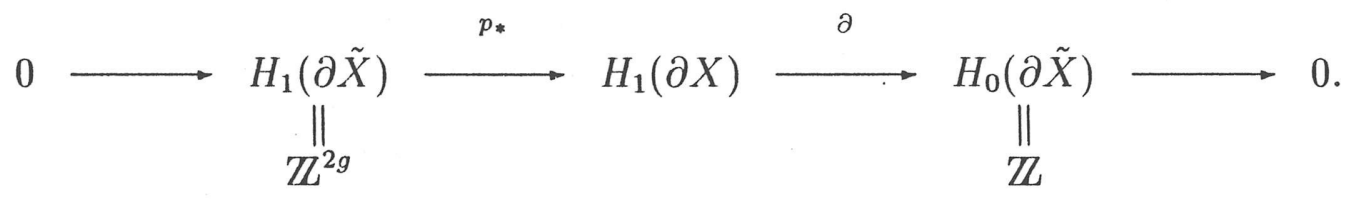

Definição 1 Diremos que um $\Lambda$-módulo $A$ é do tipo $K$ se $A$ for finitamente gerado e a múltiplicação por $(t-1)$ induz um automorfismo de $A$.

Obs: Todo módulo do tipo $K$ é de $\Lambda$-torção , ver [18].

Lema $6 H_{1}(\tilde{X})$ e $H_{1}(\tilde{X}, \partial \tilde{X})$ são $\Lambda$-módulos do tipo $K$.

Dem. Como $X$ e $\partial X$ são espaços compactos, os $\mathbb{Z}$-módulos das $q$-células de $X$ e $\partial X, C_{q}(X)$ e $C_{q}(\partial X)$ são finitamente gerados. Portanto os $\Lambda$-módulos $C_{q}(\tilde{X})$ e $C_{q}(\partial \tilde{X})$ são finitamente gerados. Como $\Lambda$ é um anel Noetheriano, $\Lambda$-submódulos e $\Lambda$-módulos quocientes de um $\Lambda$-módulo finitamente gerado são finitamente gerados. Portanto $H_{1}(\tilde{X})$ e $H_{1}(\tilde{X}, \partial \tilde{X})$ são $\Lambda$-módulos finitamente gerados.

Observemos que o final da primeira sequência do Lema 2 é:

$$
\cdots \rightarrow H_{1}(\tilde{X}) \stackrel{p_{*}}{\longrightarrow} \mathbb{Z} \stackrel{\partial}{\longrightarrow} \mathbb{Z} \stackrel{.(t-1)}{\longrightarrow} H_{0}(\tilde{X})
$$

com o último morfismo sendo nulo, o que implica $\partial u$ um isomorfismo, e portanto que $p_{*}$ é homomorfismo nulo e assim que é sobrejetor o seguinte morfismo:

$$
\cdot(t-1): H_{1}(\tilde{X}) \longrightarrow H_{1}(\tilde{X}) \text {. }
$$

Como por Lema $1 H_{1}(X, \partial X) \cong 0$ também é sobrejetor o seguinte morfismo:

$$
\cdot(t-1): H_{1}(\tilde{X}, \partial \tilde{X}) \longrightarrow H_{1}(\tilde{X}, \partial \tilde{X}) .
$$

O lema fica demonstrado ao relembrarmos os seguintes resultados de álgebra comutativa: 
- $\Lambda$ é um anel Noetheriano.

- Se $A$ é um anel Noetheriano então todo $A$-módulo finitamente gerado é um módulo Noetheriano.

- Seja $M$ um $A$-módulo Noetheriano e $u: M \rightarrow M$ um $A$-homomorfismo. Se $u$ é sobrejetivo então $u$ é um isomorfismo.

Lema 7 A aplicação $j_{*}: H_{1}(\tilde{X}) \rightarrow H_{1}(\tilde{X}, \partial \tilde{X})$ induzida pela inclusão é um isomorfismo.

Dem. Consideremos o seguinte diagrama comutativo:

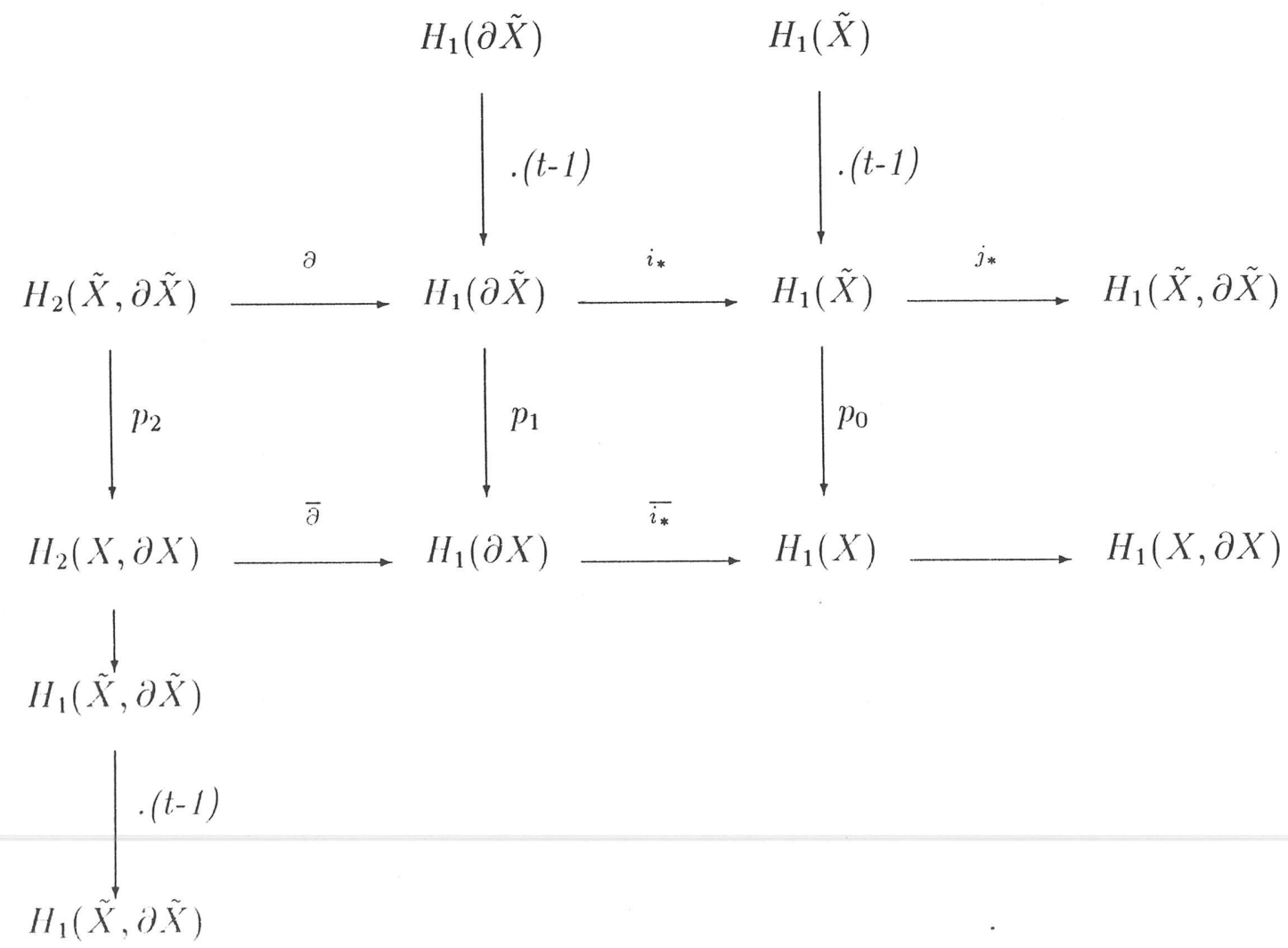

Analisando o final da sequência do $\operatorname{par}(\tilde{X}, \partial \tilde{X})$ vemos que $j_{*}$ é sobrejetora, vamos provar que $j_{*}$ é injetora. 
Observemos que $p_{2}$ é sobrejetora, $p_{1}$ é injetora e $p_{0}$ é nula. Vamos provar que $i_{*}$ é aplicação nula.

Seja $x \in \operatorname{Im}\left(i_{*}\right)$, então $p_{0}(x)=0 \in H_{1}(X)$. Seja $a \in H_{1}(\partial \tilde{X})$ tal que $i_{*}(a)=x$, temos $\overline{i_{*}}\left(p_{1}(a)\right)=0 \in H_{1}(X)$. Logo existe $b \in H_{2}(X, \partial X)$ tal que $\bar{\partial}(b)=p_{1}(a)$. Como $p_{2}$ é sobrejetora, existe $c \in H_{2}(\tilde{X}, \partial \tilde{X})$ tal que $p_{2}(c)=b$. Como $p_{1}$ é injetora, $\partial(c)=a$. Logo $x=0$.

Usaremos no Lema 9 a Sequência Espectral dos Coeficientes Universais dos recobrimentos $\tilde{X} \rightarrow X$ e $(\tilde{X}, \partial \tilde{X}) \rightarrow(X, \partial X)$. Temos:

$$
\begin{aligned}
& E_{2}^{p, q}=e^{q}\left(H_{p}(\tilde{X})\right)=\operatorname{Ext}_{\Lambda}^{q}\left[H_{p}(\tilde{X}), \Lambda\right] \\
& E_{2}^{p, q}=e^{q}\left(H_{p}(\tilde{X}, \partial \tilde{X})\right)=\operatorname{Ext}_{\Lambda}^{q}\left[H_{p}(\tilde{X}, \partial \tilde{X}), \Lambda\right]
\end{aligned}
$$

que convergem para os grupos de cohomologia equivariantes $H_{e}^{p+q}(\tilde{X})$ e $H_{e}^{p+q}(\tilde{X}, \partial \tilde{X})$ respectivamente.

No isomorfismo acima o anel $\mathbb{Z}$ é considerado como um $\Lambda$-módulo trivial, isto é, a ação do elemento " $t$ " (e também de $t^{-1}$ ) de $\Lambda$ no grupo abeliano $\mathbb{Z}$ é definida por:

$$
t: \mathbb{Z} \longrightarrow \mathbb{Z} ; t(x)=x, \forall x \in \mathbb{Z} .
$$

Seja $\lambda \in \Lambda$, usaremos a notação $\bar{\lambda}$ para indicar a imagem do elemento $\lambda$ pelo antiautomorfismo:

$$
\begin{gathered}
\phi: \Lambda \longrightarrow \Lambda ; \\
\phi\left(\sum_{i=-s}^{n} a_{i} t^{i}\right)=\sum_{i=-s}^{n} a_{i} t^{-i} .
\end{gathered}
$$

Seja $M 11 m \Lambda$-módulo a direita, usaremos a notação $\bar{M}$ para indicar que estamos considerando em $M$ uma $\Lambda$-ação a esquerda definida por:

$$
\begin{gathered}
f: \Lambda \times M \longrightarrow M \\
f(\lambda, m)=\lambda * m \stackrel{\text { def }}{=} m \bar{\lambda} .
\end{gathered}
$$


Lembremos ainda que se $M$ é um $R$-módulo a esquerda, onde $R$ é um anel e $N$ é um $R$-módulo (a esquerda ou a direita) então $\operatorname{Hom}_{R}(M, N)$ tem naturalmente uma estrutura de $R$-módulo a direita definida por:

$$
\begin{gathered}
g: R \times \operatorname{Hom}_{R}(M, N) \longrightarrow \operatorname{Hom}_{R}(M, N) ; \\
g(r, \phi)=\phi r: M \rightarrow N ; \phi r(m) \stackrel{\text { def }}{=} \phi(r m) .
\end{gathered}
$$

Quando $N$ for um $\Lambda$-módulo a esquerda usaremos a mesma notação $\bar{N}$ para indicar que estamos considerando em $N$ a estrutura de $\Lambda$-módulo a direita definida por:

$$
\begin{gathered}
h: \Lambda \times N \longrightarrow N ; \\
h(\lambda, m)=m * \lambda \stackrel{\text { def }}{=} \bar{\lambda} m .
\end{gathered}
$$

$H_{e}^{*}(\tilde{X})$ tem estrutura natural de $\Lambda$-módulo a direita. Portanto usaremos $\overline{H_{e}^{*}(\tilde{X})}$ para considerá-lo como $\Lambda$-módulo a esquerda.

Lema 8 Valem as dualidades de grupos abelianos que também são isomorfismos de $\Lambda$-módulos

$$
\overline{H_{e}^{i}(\tilde{X}, \partial \tilde{X})} \cong H_{4-i}(\tilde{X}) \text { e } \overline{H_{e}^{i}(\tilde{X})} \cong H_{4-i}(\tilde{X}, \partial \tilde{X}) \text {. }
$$

Dem. A demonstração segue do Lema de Reidemeister, ver [24].

Lema 9 Valem os isomorfismos de $\Lambda$-módulos

$$
\overline{H_{3}(\tilde{X}, \partial \tilde{X})} \cong H_{e}^{1}(\tilde{X}) \cong \operatorname{Ext}_{\Lambda}^{1}\left[H_{0}(\tilde{X}), \Lambda\right] \cong \operatorname{Ext}_{\Lambda}^{1}[\mathbb{Z}, \Lambda] \cong \mathbb{Z}
$$

Dem. Primeiramente tomamos a resolução projetiva de $\mathbb{Z} \cong H_{0}(\tilde{X})$.

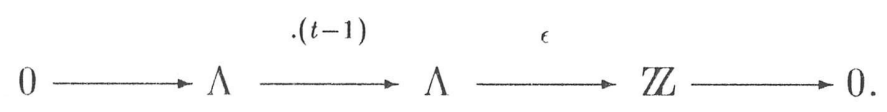

Passando o funtor Hom $(-, \Lambda)$ temos: 


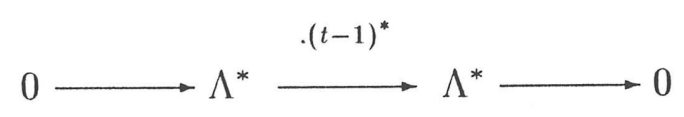

de onde temos

$$
\operatorname{Ext}_{\Lambda}^{1}\left[H_{0}(\tilde{X}), \Lambda\right] \cong \frac{\Lambda^{*}}{\operatorname{Im} \cdot(t-1)^{*}} \cong \mathbb{Z} .
$$

Portanto $\operatorname{Ext}_{\Lambda}^{1}\left[H_{0}(\tilde{X}), \Lambda\right] \cong \mathbb{Z}$.

$\operatorname{Mas} \operatorname{Ext}_{\Lambda}^{1}\left[H_{0}(\tilde{X}), \Lambda\right]=E_{2}^{0,1}$, e como a dimensão homológica de $\mathbb{Z}$ é 1 a sequência espectral dos coeficientes universais associada ao recobrimento $p: \tilde{X} \longrightarrow X$ colapsa, portanto $E_{2}^{0,1} \cong E_{\infty}^{0,1}$.

$\mathrm{Na}$ filtração

$$
H_{e}^{m}(\tilde{X})=J_{m, 0} \supset J_{m-1,1} \supset \ldots \supset J_{0, m} \supset 0
$$

obtemos

$$
H_{e}^{1}(\tilde{X})=J_{1,0} \supset J_{0,1} \supset 0
$$

$\log 0$

$$
\frac{H_{e}^{1}(\tilde{X})}{J_{0,1}} \cong E_{\infty}^{1,0} \cong E_{2}^{1,0}=E x t_{\Lambda}^{0}\left[H_{1}(\tilde{X}), \Lambda\right] \stackrel{\text { def }}{=} \operatorname{Hom}_{\Lambda}\left(H_{1}(\tilde{X}), \Lambda\right)
$$

Mas como $H_{1}(\tilde{X})$ é do tipo $K$ e portanto de $\Lambda$-torção concluimos que:

$$
\frac{H_{e}^{1}(\tilde{X})}{J_{0,1}} \cong 0
$$

Sabemos que:

$$
J_{0,1} \cong E_{\infty}^{0,1} \cong E_{2}^{0,1}=\operatorname{Ext}_{\Lambda}^{1}\left[H_{0}(\tilde{X}), \Lambda\right] \cong \mathbb{Z}
$$

Logo, por dualidade, temos

$$
\overline{H_{3}(\tilde{X}, \partial \tilde{X})} \cong H_{e}^{1}(\tilde{X}) \cong E x t_{\Lambda}^{1}\left[H_{0}(\tilde{X}), \Lambda\right] \cong \mathbb{Z}
$$

Lema 10 O homomorfismo $p_{*}: H_{3}(\tilde{X}, \partial \tilde{X}) \rightarrow H_{3}(X, \partial X)$ é um $\Lambda$-isomorfismo. O segundo módulo é gerado pela classe que tem como representante a variedade de Seifert e o primeiro módulo por uma classe que é representada por um levantamento da mesma. 
Dem. Usando excisão obtemos:

$$
H_{3}(X, \partial X) \cong H_{3}\left(S^{4}, \bar{U}\right) \cong H_{3}\left(S^{4}, L\right) \stackrel{\partial}{\longrightarrow}_{i s o} H_{2}(L)=\left\langle\left[F_{g}^{2}\right]\right\rangle
$$

Então o gerador de $H_{3}(X, \partial X)$ deve ser uma classe representada por uma 3variedade com bordo em $\partial X$, tal que a imagem do representante pelo operador bordo representa a classe geradora de $H_{2}(L)$, portanto esse gerador deve ser representado por $V^{3}$.

Tomando qualquer levantamento de $V^{3}$ vemos que este representa uma classe de $H_{3}(\tilde{X}, \partial \tilde{X})$ cuja imagem por $p_{*}$ é o gerador de $H_{3}(X, \partial X)$. Então esse levantamento representa uma classe geradora de $H_{3}(\tilde{X}, \partial \tilde{X}) \cong \mathbb{Z}$ e $p_{*}$ é um isomorfismo.

Lema 11 Para $n \geq 3, \quad H_{n}(\tilde{X}) \cong 0$.

Dem. Considere $\partial: H_{3}(\tilde{X}, \partial \tilde{X}) \rightarrow H_{2}(\partial \tilde{X})$ onde ambos os grupos são infinitos cíclicos. Vemos que a imagem de um levantamento de $V^{3}$, levantamento este que representa a classe geradora de $H_{3}(\tilde{X}, \partial \tilde{X})$, pelo operador bordo é $F_{g}^{2}$, representante da classe geradora de $H_{2}(\partial \tilde{X})$, logo $\partial$ é um isomorfismo.

Observando

$$
\cdots \rightarrow \underset{\|}{H_{3}(\partial \tilde{X})} \rightarrow H_{3}(\tilde{X}) \longrightarrow H_{3}(\tilde{X}, \partial \tilde{X}) \stackrel{\partial}{\longrightarrow} H_{2}(\partial \tilde{X})
$$

deduzimos que $H_{3}(\tilde{X}) \cong 0$.

Por dualidade temos $H_{4}(\tilde{X}) \cong \overline{H_{e}^{0}(\tilde{X}, \partial \tilde{X})} \cong 0$.

Lema 12 Se $M$ é um $\Lambda$-módulo então

$$
M \otimes_{\Lambda} \mathbb{Z} \cong[\operatorname{coker} .(t-1): M \rightarrow M] .
$$

Dem. Considere a sequência exata

$$
0 \longrightarrow \stackrel{\cdot(t-1)}{\longrightarrow} \Lambda \longrightarrow \mathbb{Z} \longrightarrow 0 .
$$


Tensorializando $\operatorname{com} M$ obtemos o segmento de sequência exata

$$
M \stackrel{\cdot(t-1)}{\longrightarrow} M \longrightarrow M \otimes_{\Lambda} \mathbb{Z} \longrightarrow 0 \text {. }
$$

Lema 13 Considere a sequência exata de $\Lambda$-módulos

$$
0 \longrightarrow M_{1} \stackrel{\phi}{\longrightarrow} M_{2} \longrightarrow \mathbb{Z}^{r} \longrightarrow 0
$$

onde $M_{2} \otimes_{\Lambda} \mathbb{Z} \cong \mathbb{Z}^{r}$. Então $\phi\left(M_{1}\right) \cong(t-1)\left[M_{2}\right]$.

Dem. Temos

$$
(t-1)\left[\frac{M_{2}}{\phi\left(M_{1}\right)}\right] \cong(t-1)\left[\mathbb{Z}^{r}\right]=0
$$

$\operatorname{logo}(t-1)\left[M_{2}\right] \subset \phi\left(M_{1}\right)$.

Também temos a sequência exata

$$
0 \rightarrow \frac{\phi\left(M_{1}\right)}{(t-1)\left[M_{2}\right]} \rightarrow \frac{M_{2}}{(t-1)\left[M_{2}\right]} \rightarrow \frac{M_{2}}{\phi\left(M_{1}\right)} \rightarrow 0 .
$$

Por Lema 12

$$
\frac{M_{2}}{(t-1)\left[M_{2}\right]} \cong M_{2} \otimes_{\Lambda} \mathbb{Z}
$$

e então usando a hipótese podemos escrever a sequência exata:

$$
0 \rightarrow \frac{\phi\left(M_{1}\right)}{(t-1)\left[M_{2}\right]} \rightarrow \mathbb{Z}^{r} \rightarrow \mathbb{Z}^{r} \rightarrow 0
$$

Então $\phi\left(M_{1}\right) \cong(t-1)\left[M_{2}\right]$.

Lema 14 Existem sequências exatas

$$
\begin{gathered}
0 \longrightarrow H_{2}(\tilde{X}) \stackrel{\cdot(t-1)}{\longrightarrow} H_{2}(\tilde{X}) \longrightarrow \mathbb{Z}^{2 g} \longrightarrow 0 \\
0 \longrightarrow H_{2}(\tilde{X}, \partial \tilde{X}) \stackrel{.(t-1)}{\longrightarrow} H_{2}(\tilde{X}, \partial \tilde{X}) \longrightarrow \mathbb{Z}^{2 g} \longrightarrow 0 \\
0 \longrightarrow H_{2}(\tilde{X}) \stackrel{j_{*}}{\longrightarrow} H_{2}(\tilde{X}, \partial \tilde{X}) \longrightarrow \mathbb{Z}^{2 g} \longrightarrow 0
\end{gathered}
$$

e a imagem de $j_{*}$ é exatamente a imagem de $.(t-1): H_{2}(\tilde{X}, \partial \tilde{X}) \rightarrow H_{2}(\tilde{X}, \partial \tilde{X})$. 
Dem. Observando que $H_{2}(X) \cong \mathbb{Z}^{2 g}, H_{2}(X, \partial X) \cong \mathbb{Z}^{2 g}, H_{3}(X) \cong 0$ e que por Lema 10 a aplicação

$$
p_{*}: H_{3}(\tilde{X}, \partial \tilde{X}) \longrightarrow H_{3}(X, \partial X)
$$

é isomorfismo, as duas primeiras sequências seguem diretamente do Lema 2 e Lema 6.

A terceira sequência vem da sequência do par $(\tilde{X}, \partial \tilde{X})$. Basta observarmos que

$$
H_{3}(\tilde{X}, \partial \tilde{X}) \stackrel{\partial}{\longrightarrow} H_{2}(\partial \tilde{X})
$$

é um isomorfismo pela demonstração do Lema 11, que

$$
H_{1}(\tilde{X}) \stackrel{j_{*}}{\longrightarrow} H_{1}(\tilde{X}, \partial \tilde{X})
$$

é um isomorfismo por Lema 7 e que

$$
H_{1}(\partial \tilde{X}) \cong \mathbb{Z}^{2 g}
$$

Pelo Lema 12

$$
H_{2}(\tilde{X}, \partial \tilde{X}) \otimes_{\Lambda} \mathbb{Z} \cong\left[\text { coker. }(t-1): H_{2}(\tilde{X}, \partial \tilde{X}) \longrightarrow H_{2}(\tilde{X}, \partial \tilde{X})\right] \cong \mathbb{Z}^{2 g}
$$

Aplicando então o Lema 13 obtemos

$$
\operatorname{Im}\left(j_{*}\right) \cong(t-1)\left[H_{2}(\tilde{X}, \partial \tilde{X})\right] .
$$

OBS: Usaremos a notação $M^{*}$ para indicar $\operatorname{Hom}_{\Lambda}(M, \Lambda)$.

Lema 15 Existem duas sequências exatas de $\Lambda$-módulos:

A)

$$
\begin{aligned}
& 0 \rightarrow e^{1}\left[H_{1}(\tilde{X})\right] \rightarrow \overline{H_{2}(\tilde{X}, \partial \tilde{X})} \stackrel{\psi}{\longrightarrow} H_{2}(\tilde{X})^{*} \stackrel{d}{\longrightarrow} e^{2}\left[H_{1}(\tilde{X})\right] \rightarrow \\
& \overline{H_{1}(\tilde{X}, \partial \tilde{X})} \rightarrow e^{1}\left[H_{2}(\tilde{X})\right] \rightarrow 0 \\
& \text { B) } \\
& 0 \rightarrow e^{1}\left[H_{1}(\tilde{X}, \partial \tilde{X})\right] \rightarrow \overline{H_{2}(\tilde{X})} \stackrel{\phi}{\longrightarrow} H_{2}(\tilde{X}, \partial \tilde{X})^{*} \stackrel{d}{\longrightarrow} \\
& e^{2}\left[H_{1}(\tilde{X}, \partial \tilde{X})\right] \rightarrow \overline{H_{1}(\tilde{X})} \rightarrow e^{1}\left[H_{2}(\tilde{X}, \partial \tilde{X})\right] \rightarrow 0
\end{aligned}
$$


Dem. Vamos fazer a demonstração da primeira sequência pois a demonstração da segunda sequência é análoga. Consideremos a Sequência Espectral dos Coeficientes Universais.

$$
E_{2}^{p, q}=e^{q}\left[H_{p}(\tilde{X})\right]=\operatorname{Ext}_{\Lambda}^{q}\left[H_{p}(\tilde{X}), \Lambda\right] \text { converge para } H_{e}^{*}(\tilde{X}) \text {, com a diferencial }
$$
tendo bigrau $(1-r, r)$.

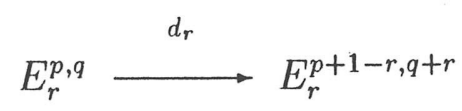

A única diferencial $d_{r}(r \geq 2)$ que pode ser não nula é

$$
d_{2}: E_{2}^{2,0} \longrightarrow E_{2}^{1,2}
$$

pois $H_{p}(\tilde{X}) \cong 0$ para $p \geq 3$ e a dimensão homológica de $\Lambda$ é dois, o que ocasiona $E_{2}^{p, q} \cong 0$ se $q \geq 3$.

$$
H_{0}(\tilde{X}) \cong \mathbb{Z} \operatorname{logo} e^{0}\left[H_{0}(\tilde{X})\right]=\operatorname{Hom}_{\Lambda}(\mathbb{Z}, \Lambda)=H_{0}(\tilde{X})^{*} \cong 0 .
$$
0.

$H_{1}(\tilde{X})$ é do tipo $K$ e portanto de $\Lambda$-torção logo $e^{0}\left[H_{1}(\tilde{X})\right] \cong \operatorname{Hom}_{\Lambda}\left[H_{1}(\tilde{X}), \Lambda\right]=$ $e^{1}\left[H_{0}(\tilde{X})\right]=\operatorname{Ext}_{\Lambda}^{1}\left[H_{0}(\tilde{X}), \Lambda\right]=\operatorname{Ext}_{\Lambda}^{1}[\mathbb{Z}, \Lambda] \cong[\operatorname{coker} .(t-1): \Lambda \rightarrow \Lambda] \cong \mathbb{Z}$.

$e^{2}\left[H_{0}(\tilde{X})\right] \cong 0$ pois a dimensão homológica de $\mathbb{Z}$ é igual a 1 .

$e^{2}\left[H_{2}(\tilde{X})\right]$ também é nulo pois

$0 \cong H_{e}^{4}(\tilde{X})=J_{4,0} \supset J_{3,1} \supset J_{2,2} \supset J_{1,3} \supset J_{0,4} \supset 0$.

Temos $E_{2}^{2,2}=E_{\infty}^{2,2}=\frac{J_{2,2}}{J_{1,3}}$. 
Os termos $E_{2}^{r, s}$ são apresentados no quadro abaixo (os demais são nulos).

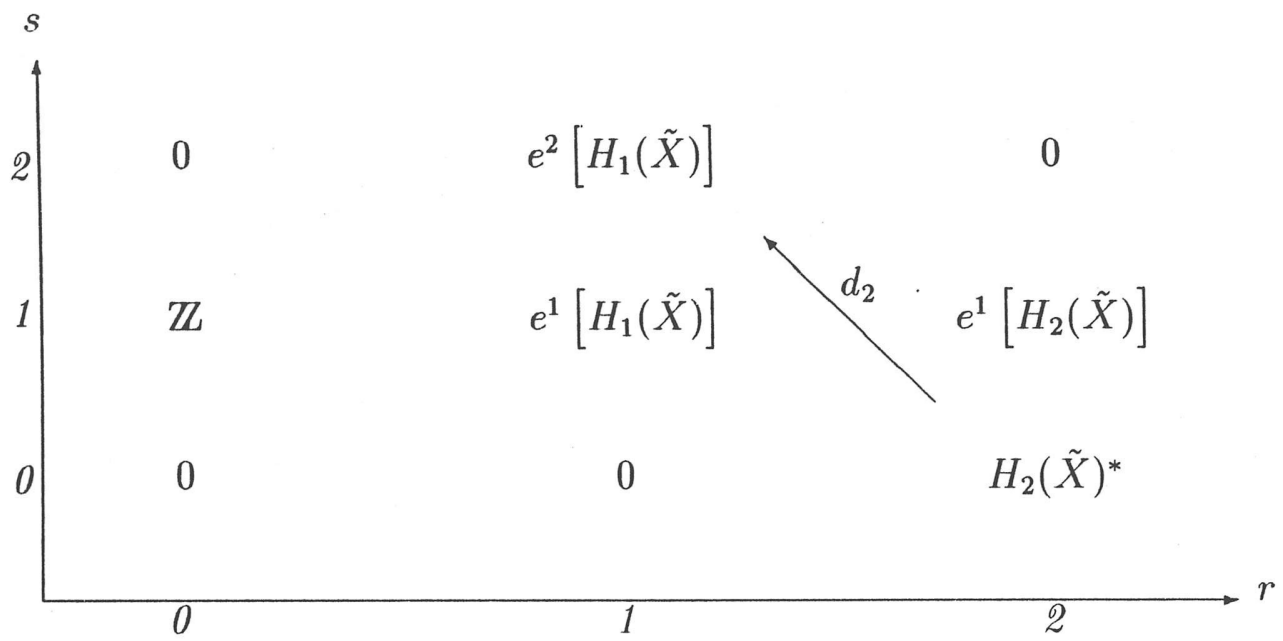

A diferencial citada anteriormente como possivelmente não nula é

$$
d_{2}: H_{2}(\tilde{X})^{*} \longrightarrow e^{2}\left[H_{1}(\tilde{X})\right]
$$

Vamos analisar $H_{e}^{3}(\tilde{X})$ e $H_{e}^{2}(\tilde{X})$ :

Tome a filtração

$$
H_{e}^{3}(\tilde{X})=J_{3,0} \supset J_{2,1} \supset J_{1,2} \supset J_{0,3} \supset 0 \text {. }
$$

Temos

$$
E_{\infty}^{0,3} \cong 0 \cong E_{\infty}^{3,0} \log \mathrm{o} \quad J_{3,0}=J_{2,1} \text { e } J_{0,3}=0
$$

resultando

$$
H_{e}^{3}(\tilde{X}) \supset J_{1,2} \supset 0
$$


Temos

$$
\begin{gathered}
E_{\infty}^{1,2}=E_{3}^{1,2}=\left[\operatorname{coker}\left(d_{2}\right): H_{2}(\tilde{X})^{*} \rightarrow e^{2}\left[H_{1}(\tilde{X})\right]\right] \\
E_{\infty}^{2,1}=E_{2}^{2,1}=e^{1}\left[H_{2}(\tilde{X})\right] .
\end{gathered}
$$

Obtemos a sequência exata

$$
0 \rightarrow J_{1,2} \rightarrow H_{e}^{3}(\tilde{X}) \rightarrow \frac{H_{e}^{3}(\tilde{X})}{J_{1,2}} \rightarrow 0
$$

que na verdade é

$$
0 \rightarrow \operatorname{coker}\left(d_{2}\right) \rightarrow H_{e}^{3}(\tilde{X}) \rightarrow e^{1}\left[H_{2}(\tilde{X})\right] \rightarrow 0
$$

Tome a filtração

$$
H_{e}^{2}(\tilde{X})=J_{2,0} \supset J_{1,1} \supset J_{0,2} \supset 0 .
$$

Temos

$$
E_{\infty}^{0,2}=E_{2}^{0,2}=e^{2}\left[H_{0}(\tilde{X})\right]=0 .
$$

Então a filtração fica apenas

$$
H_{e}^{2}(\tilde{X}) \supset J_{1,1} \supset 0 .
$$

Mas

$$
\begin{gathered}
E_{\infty}^{2,0}=E_{3}^{2,0}=\operatorname{ker}\left(d_{2}\right), \quad E_{\infty}^{2,0}=\frac{H_{e}^{2}(\tilde{X})}{J_{1,1}} \\
J_{1,1}=E_{\infty}^{1,1}=E_{2}^{1,1}=e^{1}\left[H_{1}(\tilde{X})\right]
\end{gathered}
$$

E assim obtemos a sequência exata

$$
0 \rightarrow J_{1,1} \rightarrow H_{e}^{2}(\tilde{X}) \rightarrow \frac{H_{e}^{2}(\tilde{X})}{J_{1,1}} \rightarrow 0
$$

que na verdade é 


$$
0 \rightarrow e^{1}\left[H_{1}(\tilde{X})\right] \rightarrow H_{e}^{2}(\tilde{X}) \rightarrow \operatorname{ker}\left(d_{2}\right) \rightarrow 0
$$

Colocando juntas as duas sequências obtidas conseguimos

$$
\begin{aligned}
& 0 \rightarrow e^{1}\left[H_{1}(\tilde{X})\right] \rightarrow H_{e}^{2}(\tilde{X}) \rightarrow H_{2}(\tilde{X})^{*} \stackrel{d_{2}}{\longrightarrow} e^{2}\left[H_{1}(\tilde{X})\right] \rightarrow \\
& H_{e}^{3}(\tilde{X}) \rightarrow e^{1}\left[H_{2}(\tilde{X})\right] \rightarrow 0 .
\end{aligned}
$$

Usando dualidades podemos escrever

$$
\begin{aligned}
& 0 \longrightarrow e^{1}\left[H_{1}(\tilde{X})\right] \rightarrow \overline{H_{2}(\tilde{X}, \partial \tilde{X})} \stackrel{\psi}{\longrightarrow} H_{2}(\tilde{X})^{*} \stackrel{d}{\longrightarrow} e^{2}\left[H_{1}(\tilde{X})\right] \rightarrow \\
& \overline{H_{1}(\tilde{X}, \partial \tilde{X})} \rightarrow e^{1}\left[H_{2}(\tilde{X})\right] \rightarrow 0 .
\end{aligned}
$$


Lema $16 \operatorname{rank}_{\Lambda} H_{2}(\tilde{X})=2 g, \quad \operatorname{rank}_{\Lambda} H_{2}(\tilde{X}, \partial \tilde{X})=2 g$.

Dem. Já foram calculados os seguintes isomorfismos

$$
H_{0}(X) \cong \mathbb{Z}, \quad H_{1}(X) \cong \mathbb{Z}, \quad H_{2}(X) \cong \mathbb{Z}^{2 g}, \quad H_{3}(X) \cong 0, \text { e } \quad H_{4}(X) \cong 0
$$

Logo a característica de Euler de $X$ é:

$$
\mathcal{X}(X)=\sum_{q=0}^{4}(-1)^{q} \operatorname{rank}_{\mathbb{Z}}\left(H_{q}(X)\right)=2 g .
$$

Por teorema 4.3.14 de Spanier [35] temos que a característica de Euler de um complexo de cadeias é igual a característica de Euler da homologia deste complexo de cadeias.

Como $X$ é um $C W$-complexo de dimensão 4 denotamos por $C_{q}(X)$ o $\mathbb{Z}$-módulo das $q$-ésimas células de X. Podemos escrever a igualdade:

$$
\sum_{q=0}^{4}(-1)^{q} \operatorname{rank}_{\mathbb{Z}}\left(C_{q}(X)\right)=2 g
$$

Sabemos que $C_{q}(\tilde{X})$ é um $\Lambda$-módulo finitamente gerado para $q=1, \ldots, 4$ e que vale a igualdade

$$
\operatorname{rank}_{\Lambda}\left(C_{q}(\tilde{X})\right)=\operatorname{rank}_{\mathbb{Z}}\left(C_{q}(X)\right)
$$

Logo deduzimos que

$$
2 g=\sum_{q=0}^{4}(-1)^{q} \operatorname{rank}_{\Lambda}\left(C_{q}(\tilde{X})\right)=\sum_{q=0}^{4}(-1)^{q} \operatorname{rank}_{\Lambda}\left(H_{q}(\tilde{X})\right) .
$$

Mas

$$
\operatorname{rank}_{\Lambda}\left(H_{0}(\tilde{X})\right)=\operatorname{rank}_{\Lambda}(\mathbb{Z})=0
$$

$H_{1}(\tilde{X})$ é do tipo $K$ e portanto de $\Lambda$-torção, logo $\operatorname{rank}_{\Lambda}\left(H_{1}(\tilde{X})\right)=0$. 
E finalmente por Lema 11 temos

$$
\operatorname{rank}_{\Lambda}\left(H_{3}(\tilde{X})\right)=\operatorname{rank}_{\Lambda}\left(H_{4}(\tilde{X})\right)=0 \text {. }
$$

Logo

$$
2 g=\operatorname{rank}_{\Lambda} H_{2}(\tilde{X}) .
$$

De modo semelhante prova-se $\operatorname{rank}_{\Lambda} H_{0}(\tilde{X}, \partial \tilde{X})=0$ e $\operatorname{rank}_{\Lambda} H_{1}(\tilde{X}, \partial \tilde{X})=0$.

Por Lema $9 \operatorname{rank}_{\Lambda} H_{3}(\tilde{X}, \partial \tilde{X})=0$.

Por Lema $8 \operatorname{rank}_{\Lambda} H_{4}(\tilde{X}, \partial \tilde{X})=\operatorname{rank}_{\Lambda} H_{e}^{0}(\tilde{X})=0$.

Portanto $2 g=\operatorname{rank}_{\Lambda} H_{2}(\tilde{X}, \partial \tilde{X})$.

OBS. A segunda igualdade do Lema 16 é o resultado 3.3 de Sekine [31].

As seguintes definições encontram-se em N. Bourbaki, "Élements de Mathématiques, XXVII, Algébre Commutative", Hermann, Paris, 1968.

Definição 2 Seja $M$ um $\Lambda$-módulo, diremos que $x \in M$ é um elemento pseudonulo se $\operatorname{Ann}(x)=\{y \in \Lambda \mid y x=0\}$ não estiver contido em nenhum ideal principal próprio de $\Lambda$.

Definição 3 Denotamos por NM o submódulo dos pseudonulos de $M$, isto é, $N M=\{x \in M \mid A n n(x) \not \subset$ ideal principal próprio $\}$.

Definição 4 Denotamos por $F N M=\frac{M}{N M}$ a parte livre de pseudonulos de $M$.

Definição 5 Denotamos a parte de $\Lambda$-torção de $M\{x \in M \mid \exists \lambda \neq 0 \in \Lambda, \lambda x=0\}$ por $T(M)$ e a parte de $\mathbb{Z}$-torção de $M$ por $t(M)$.

Lema $17 H_{2}(\tilde{X})$ e $H_{2}(\tilde{X}, \partial \tilde{X})$ são livres de pseudo-nulos.

Dem. Basta mostrar que a parte de $\Lambda$-torção de $\overline{H_{2}(\tilde{X})}$ denotada por $T\left[\overline{H_{2}(\tilde{X})}\right]$ é livre de pseudo-nulos.

Temos 


$$
T\left[\overline{H_{2}(\tilde{X})}\right]=e^{1}\left[H_{1}(\tilde{X}, \partial \dot{\tilde{X}})\right]
$$

A igualdade vem da sequência exata

$$
0 \rightarrow e^{1}\left[H_{1}(\tilde{X}, \partial \tilde{X})\right] \rightarrow \overline{H_{2}(\tilde{X})} \rightarrow \operatorname{Im}(\phi) \subset H_{2}(\tilde{X}, \partial \tilde{X})^{*}
$$

Pelo corolário 1.10 de [26] sabemos que para qualquer $\Lambda$-módulo $M, M^{*}$ é $\Lambda$-livre, logo

$$
T\left[\overline{H_{2}(\tilde{X})}\right] \subset e^{1}\left[H_{1}(\tilde{X}, \partial \tilde{X})\right]
$$

Se $M$ é um $\Lambda$-módulo do tipo $K$ então $e^{1}(M)$ é ainda um $\Lambda$-módulo do tipo $K$ (usa-se o fato de que $\Lambda$ é Noetheriano e a definição de $e^{1}(-)$ ) e portanto de $\Lambda$-torção , logo

$$
e^{1}\left[H_{1}(\tilde{X}, \partial \tilde{X})\right] \subset T\left[\overline{H_{2}(\tilde{X})}\right]
$$

Concluimos agora que $e^{1}\left[H_{1}(\tilde{X}, \partial \tilde{X})\right]$ é livre de pseudo-nulo usando a proposição 1.8 de [26] que demonstra serem livres de pseudo-nulos os módulos $e^{1}[T]$, onde $T$ é módulo de $\Lambda$-torção .

Portanto $\mathrm{NH}_{2}(\tilde{X})=0$.

Analogamente prova-se que $\mathrm{NH}_{2}(\tilde{X}, \partial \tilde{X})=0$. 


\section{Teoremas sobre Módulos de Alexander}

Usando as Proposições 4.1 e 4.2 de [18] e 1.4(b) e 1.4(c) de [26] temos os seguintes $\Lambda$-isomorfismos:

$$
\begin{aligned}
& e^{1}\left[H_{1}(\tilde{X})\right] \cong \operatorname{Hom}_{\Lambda}\left(F N H_{1}(\tilde{X}), \frac{Q(\Lambda)}{\Lambda}\right) \\
& e^{1}\left[H_{1}(\tilde{X}, \partial \tilde{X})\right] \cong \operatorname{Hom}_{\Lambda}\left(F N H_{1}(\tilde{X}, \partial \tilde{X}), \frac{Q(\Lambda)}{\Lambda}\right), \\
& e^{2}\left[H_{1}(\tilde{X})\right] \cong \operatorname{Hom}_{\mathbb{Z}}\left(N H_{1}(\tilde{X}), \frac{Q}{\mathbb{Z}}\right), \\
& e^{2}\left[H_{1}(\tilde{X}, \partial \tilde{X})\right] \cong \operatorname{Hom}_{\mathbb{Z}}\left(N H_{1}(\tilde{X}, \partial \tilde{X}), \frac{Q}{\mathbb{Z}}\right) .
\end{aligned}
$$

Usando os resultados já demonstrados temos os isomorfismos de $\Lambda$-módulos a direita:

$$
\begin{aligned}
& \overline{T\left(H_{2}(\tilde{X}, \partial \tilde{X})\right)} \stackrel{\Omega}{\longrightarrow} \operatorname{Hom}_{\Lambda}\left(F N H_{1}(\tilde{X}), \frac{Q(\Lambda)}{\Lambda}\right), \\
& \overline{T\left(H_{2}(\tilde{X})\right)} \stackrel{\Theta}{\longrightarrow} \operatorname{Hom}_{\Lambda}\left(F N H_{1}(\tilde{X}, \partial \tilde{X}), \frac{Q(\Lambda)}{\Lambda}\right) .
\end{aligned}
$$

E os monomorfismos de $\Lambda$-módulos a direita:

$$
\begin{gathered}
\frac{H_{2}(\tilde{X})^{*}}{\operatorname{Im}(\psi)} \stackrel{\tau}{\longrightarrow} \operatorname{Hom}_{\mathbb{Z}}\left(N H_{1}(\tilde{X}), \frac{Q}{\mathbb{Z}}\right), \\
\frac{H_{2}(\tilde{X}, \partial \tilde{X})^{*}}{\operatorname{Im}(\phi)} \stackrel{\gamma}{\longrightarrow} \operatorname{Hom}_{\mathbb{Z}}\left(N H_{1}(\tilde{X}, \partial \tilde{X}), \frac{Q}{\mathbb{Z}}\right) .
\end{gathered}
$$

Portanto podemos definir os pairings:

$$
\begin{gathered}
<,>: T\left(H_{2}(\tilde{X}, \partial \tilde{X})\right) \times F N H_{1}(\tilde{X}) \longrightarrow \frac{Q(\Lambda)}{\Lambda} \\
<\alpha, \beta>=[\Omega(\bar{\alpha})](\beta)
\end{gathered}
$$

e 


$$
\begin{gathered}
<,>: T\left(H_{2}(\tilde{X})\right) \times F N H_{1}(\tilde{X}, \partial \tilde{X}) \longrightarrow \frac{Q(\Lambda)}{\Lambda} \\
<a, b>=[\Theta(\bar{a})](b)
\end{gathered}
$$

que satisfazem as propriedades:

a) não singulares

A adjunta de $<,>$, que é um homomorfismo

$$
\overline{T\left(H_{2}(\tilde{X}, \partial \tilde{X})\right)} \longrightarrow \operatorname{Hom}_{\Lambda}\left(F N H_{1}(\tilde{X}), \frac{Q(\Lambda)}{\Lambda}\right)
$$

é um isomorfismo.

O mesmo vale para $H_{2}(\tilde{X})$.

b) conjugada-linear

$$
<\lambda \alpha, \beta>=\bar{\lambda}<\alpha, \beta>=<\alpha, \bar{\lambda} \beta>, \quad \forall \lambda \in \Lambda .
$$

Também podemos definir os pairings:

$$
\begin{gathered}
{[,]: \frac{\overline{H_{2}(\tilde{X})^{*}}}{\operatorname{Im}(\psi)} \times N H_{1}(\tilde{X}) \longrightarrow \frac{Q}{\mathbb{Z}},} \\
{[[r], s]=[\tau([\bar{r}])](s)}
\end{gathered}
$$

e

$$
\begin{gathered}
{[,]: \frac{\overline{H_{2}(\tilde{X}, \partial \tilde{X})^{*}}}{\operatorname{Im}(\phi)} \times N H_{1}(\tilde{X}, \partial \tilde{X}) \longrightarrow \frac{Q}{\mathbb{Z}}} \\
{[[t], e]=[\gamma([t])](e)}
\end{gathered}
$$

que satisfazem as propriedades: 
a) não-degenerados

A adjunta de [,], que é um homomorfismo

$$
\frac{H_{2}(\tilde{X})^{*}}{\operatorname{Im}(\psi)} \longrightarrow \operatorname{Hom}_{\mathbb{Z}}\left(N H_{1}(\tilde{X}), \frac{Q}{\mathbb{Z}}\right)
$$

é um monomorfismo. O mesmo vale para $H_{2}(\tilde{X}, \partial \tilde{X})^{*}$.

b) conjugada-autoadjunta e $\mathbb{Z}$-linear

$$
\begin{gathered}
{[l r, s]=[r, \bar{l} s], \quad \forall l \in \Lambda,} \\
{[z r, s]=z[r, s]=[r, z s], \quad \forall z \in \mathbb{Z} .}
\end{gathered}
$$

Também podemos definir pairings possivelmente degenerados

$$
\begin{gathered}
{[,]_{1}: \overline{H_{2}(\tilde{X})^{*}} \times N H_{1}(\tilde{X}) \longrightarrow \frac{Q}{\mathbb{Z}},} \\
{[r, s]_{1}=[[r], s]}
\end{gathered}
$$

$\mathrm{e}$

$$
\begin{gathered}
{[,]_{2}: \overline{H_{2}(\tilde{X}, \partial \tilde{X})^{*}} \times N H_{1}(\tilde{X}, \partial \tilde{X}) \longrightarrow \frac{Q}{\mathbb{Z}},} \\
{[t, l]_{2}=[[t], l] .}
\end{gathered}
$$

Podemos agora enunciar os teoremas principais desta seção .

Teorema 1 Seja $\tilde{X} \rightarrow X$ como antes, então:

1) $H_{1}(\tilde{X})$ e $H_{1}(\tilde{X}, \partial \tilde{X})$ são $\Lambda$-módulos do tipo $K$.

2) $\operatorname{rank}_{\Lambda} H_{2}(\tilde{X})=2 g=\operatorname{rank}_{\Lambda} H_{2}(\tilde{X}, \partial \tilde{X})$.

3) $H_{2}(\tilde{X}) \otimes_{\Lambda} \mathbb{Z} \cong \mathbb{Z}^{2 g} \cong H_{2}(\tilde{X}, \partial \tilde{X}) \otimes_{\Lambda} \mathbb{Z}$. 
4) existem sequências exatas

$$
\begin{aligned}
& 0 \rightarrow e^{1}\left[H_{1}(\tilde{X})\right] \rightarrow \overline{H_{2}(\tilde{X}, \partial \tilde{X})} \stackrel{\psi}{\longrightarrow} H_{2}(\tilde{X})^{*} \stackrel{d}{\longrightarrow} \\
& e^{2}\left[H_{1}(\tilde{X})\right] \rightarrow \overline{H_{1}(\tilde{X}, \partial \tilde{X})} \rightarrow e^{1}\left[H_{2}(\tilde{X})\right] \rightarrow 0, \\
& 0 \rightarrow e^{1}\left[H_{1}(\tilde{X}, \partial \tilde{X})\right] \rightarrow \overline{H_{2}(\tilde{X})} \stackrel{\phi}{\longrightarrow} H_{2}(\tilde{X}, \partial \tilde{X})^{*} \longrightarrow d \\
& e^{2}\left[H_{1}(\tilde{X}, \partial \tilde{X})\right] \rightarrow \overline{H_{1}(\tilde{X})} \rightarrow e^{1}\left[H_{2}(\tilde{X}, \partial \tilde{X})\right] \rightarrow 0 .
\end{aligned}
$$

Teorema 2 Existe $<,>: T\left(H_{2}(\tilde{X}, \partial \tilde{X})\right) \times F N H_{1}(\tilde{X}) \rightarrow Q(\Lambda) / \Lambda$ que é conjugadalinear e não-singular.

OBS: Akio Kawauchi no seu trabalho "Three Dualities on the Integral Homology of Infinite Cyclic Coverings of Manifolds" publicado em Osaka J. Math. 23(1986), pag. 633-651, exibe resultado igual ao teorema acima utilizando outros métodos.

Teorema 3 Existe [,] : $\overline{\left(H_{2}(\tilde{X})^{*} / \operatorname{Im}(\psi)\right)} \times N H_{1}(\tilde{X}) \rightarrow Q / \mathbb{Z}$ que é conjugadaautoadjunta, não-degenerado e $\mathbb{Z}$-linear.

Teorema 4 Dados $\Lambda$-módulos $A$ e $B$ satisfazendo:

1. A é $\Lambda$-módulo do tipo $K$,

2. $\operatorname{rank}_{\Lambda} B=2 g$,

3. $B \otimes{ }_{\Lambda} \mathbb{Z} \cong \mathbb{Z}^{2 g}$,

1. existe sequência exata

$$
0 \rightarrow e^{1}(A) \rightarrow \bar{B} \rightarrow B^{*} \rightarrow e^{2}(A) \rightarrow \bar{A} \rightarrow e^{1}(B) \rightarrow 0 e,
$$

5. a parte de Zh-torção de $A$ é nula, isto é, $t(A)=0$.

Entâo existe um mergulho $f: F_{g}^{2} \rightarrow S^{4}$ tal que $H_{1}(\tilde{X}) \cong A$ e $H_{2}(\tilde{X}) \cong B$. 
Demonstração do Teorema 4.

Com a hipótese adicional $t(A)=0$ obtemos através da Proposição 1.19 de [26] que $N(A)=0=e^{2}(A)$.

Então a sequência do item 4 do Teorema 4 torna-se

$$
0 \rightarrow e^{1}(A) \rightarrow \bar{B} \rightarrow B^{*} \rightarrow 0
$$

com $B^{*} \cong \Lambda^{2 g}$ que é obviamente $\Lambda$-livre e portanto a sequência cinde, e obtemos $\bar{B} \cong \Lambda^{2 g} \oplus e^{1}(A)$,onde $e^{1}(A)=T(\bar{B})$ a parte de $\Lambda$-torção de $\bar{B}$ e portanto $T(B)$ é também do tipo $K$.

Considerando agora o par $A$ e $T(B)$ observamos que eles satisfazem todas as condições para serem realizados como módulos de Alexander de um nó $S^{2} \rightarrow S^{4}$ como provado por Levine [18] no Teorema 11.1 .

Podemos agora utilizar uma das realizações $S$ de Levine, com $H_{1}\left(\tilde{X}_{S}\right) \cong A$ e $H_{2}\left(\tilde{X}_{S}\right) \cong T(B)$; Devemos fazer uma soma conexa $R=S \star T$ com uma superfície orientada fechada $T$ trivialmente mergulhada em $S^{4}$ de genus " $g$ ", (isto é, $T$ é bordo de um "handlebody" sólido de genus $g$ ). Conhecemos o fato de que o complementar $S^{4}$ - T tem o mesmo tipo de homotopia que um "wedge" formado por uma esfera $S^{1}$ e por $2 g$ esferas $S^{2}$, então temos $H_{1}\left(\tilde{X}_{T}\right) \cong 0$ e $H_{2}\left(\tilde{X}_{T}\right) \cong \Lambda^{2 g}$ e valem as relações

$$
H_{1}\left(\tilde{X}_{R}\right) \cong H_{1}\left(\tilde{X}_{S}\right) \oplus H_{1}\left(\tilde{X}_{T}\right)
$$

e

$$
H_{2}\left(\tilde{X}_{R}\right) \cong H_{2}\left(\tilde{X}_{S}\right) \oplus H_{2}\left(\tilde{X}_{T}\right)
$$




\section{Nós Satélites}

Ao estudarmos "Teoria de Nós Clássica", isto é, estudo de mergulhos da esfera $S^{1}$ na esfera $S^{3}$, encontramos uma noção de proximidade entre imagens desses mergulhos que não é a que temos quando pensamos em homotopia. Essa idéia é a de nó satélite.

Se imaginarmos que a imagem $\Gamma$ de um mergulho como acima é a trajetória de uma partícula $r$ no espaço $S^{3}$ e admitirmos que existe uma outra partícula $s$ orbitando em torno de $r$. A medida que $r$ percorre sua trajetória, $s$ irá descrever uma curva próxima da descrita por $r$. Algo como a trajetória que a Lua perfaz enquanto a Terra segue sua órbita em torno do Sol.

Se a trajetória $\Sigma$ percorrida pela partícula $s$ for uma curva fechada, teremos a noção de curva satélite; diremos então que $\Sigma$ é uma curva satélite de $\Gamma$.

Vê-se portanto que a curva $\Sigma$ tem uma proximidade geométrica com $\Gamma$. Podese perguntar se essa proximidade geométrica reflete-se em alguma proximidade algébrica entre invariantes que podem ser calculados para as curvas $\Gamma$ e $\Sigma$. Ou melhor, pode-se perguntar que noção de proximidade algébrica nós temos quando observamos dois objetos de uma mesma categoria algébrica obtidos através de $\mathrm{um}$ funtor avaliado em uma curva $\Gamma$ e em sua curva satélite $\Sigma$ ?

Vejamos.

Considere o nó trivial $K^{*} \subset S^{3}$ e seja $V^{*}$ uma vizinhança tubular de $K^{*}$. Seja $E$ um nó contido em $V^{*}$. Então $E$ é homólogo em $V^{*}$ a algum múltiplo de $K^{*}$, digamos

$$
E \sim n . K^{*} \mathrm{em} V^{*}
$$

Redefinindo a orientação de $E$ podemos ter $n \geq 0$.

Tome agora um difeomorfismo $\phi: V^{*} \rightarrow V$ que leva $V^{*}$ em uma vizinhança tubular $V$ de um nó $K \subset S^{3}$ e seja $L=\phi(E)$.

Seifert em [30] provou os seguintes resultados.

Teorema I: Para $n=0$ os invariantes homológicos de $L$ e de $E$ são os mesmos.

Teorema II: Entre os polinômios de Alexander $\Delta_{L}(x), \Delta_{E}(x)$ e $\Delta_{K}(x)$ dos nós $L, E$ e $K$ respectivamente vale a seguinte equação

$$
\Delta_{L}(x)=\Delta_{E}(x) \cdot \Delta_{K}\left(x^{n}\right) .
$$

Para a "Teoria de Nós Generalizada", isto é, estudo de mergulhos de $S^{k}$ em $S^{k+2}$, 
Milnor em [23] define o q-ésimo polinômio de Alexander $\Delta_{K}^{q}(t)$ de um nó $K$ contido em $S^{k+2}$ como o "order" do $Q\left[t, t^{-1}\right]$-módulo $H_{q}(\tilde{X} ; Q)$, onde $\tilde{X}$ é o espaço total do recobrimento cíclico infinito do complementar do nó $K$.

Em dimensões mais altas temos situação similar.

Seja $K^{*}$ o nó trivial em $S^{k+2}$ e $V^{*}$ uma vizinhança tubular, seja $E$ um nó contido em $V^{*}$. Seja $\phi: V^{*} \rightarrow V$ um difeomorfismo entre $V^{*}$ e uma vizinhança tubular de um nó $K$ em $S^{k+2}$ e seja $L=\phi(E)$. Redefinindo a orientação de $E$ temos que $E$ é homólogo em $V^{*}$ a algum múltiplo inteiro não negativo de $K^{*}$, isto é, $E \sim \lambda . K^{*}, \lambda \geq 0$.

Usando a definição acima, Shinohara Y. em [33] provou o seguinte teorema.

Teorema 1.1: Para $1 \leq q \leq n$ vale $\Delta_{L}^{q}(t)=\Delta_{E}^{q}(t) \cdot \Delta_{K}^{q}\left(t^{\lambda}\right)$.

Procuramos agora generalizar o conceito de nós satélites para variedades e estudar as relações entre os módulos de Alexander de um mergulho em codimensão dois e seus possíveis mergulhos satélites. 


\section{Construção da Variedade Satélite}

\subsection{Espaços de Configuração}

Denotemos por $\prod_{i=1}^{n} M$ o produto cartesiano de $n$ folhas iguais a $M$ e denote

$$
F_{0, n} M=\left\{\left(z_{1}, \ldots, z_{n}\right) \in \prod_{i=1}^{n} M \mid z_{i} \neq z_{j} \text { se } i \neq j\right\} .
$$

Como $F_{0, n} M$ é um subconjunto aberto de uma variedade é também uma variedade. O grupo $\pi_{1}\left(F_{0, n} M\right)$ é dito o grupo das tranças puras com $n$ cordas da variedade $M$.

Coloquemos em $F_{0, n} M$ a seguinte relação de equivalência: Dois pontos $z$ e $z^{\prime}$ $\in F_{0, n} M$ são ditos equivalentes se as coordenadas $\left(z_{1}, \ldots, z_{n}\right)$ de $z$ diferem das coordenadas $\left(z_{1}^{\prime}, \ldots, z^{\prime}{ }_{n}\right)$ de $z^{\prime}$ por uma permutação. Seja $B_{0, n} M$ o espaço de identificação de $F_{0, n} M$ sob esta relação de equivalência. Este espaço é também uma variedade de dimensão m.n. O grupo $\pi_{1}\left(B_{0, n} M\right)$ é dito o grupo das tranças totais de $M$.

Denotando o grupo de permutações em $n$ elementos por $\Sigma_{n}$ podemos escrever:

$$
B_{0, n} M=\frac{F_{0, n} M}{\sum_{n}}
$$

Podemos chamar a atenção ao fato de identificarmos um elemento $x=\left[x_{1}, \ldots, x_{n}\right] \in$ $B_{0, n}\left(\right.$ int $\left.D^{2}\right)$ com o conjunto

$$
\left\{x_{1}, \ldots, x_{n} ; x_{i} \in \operatorname{int} D^{2}, i=1, \ldots, n\right\}
$$

de pontos de int $D^{2}$.

() "grupo das tranças" clássico de Artin [1] é o grupo $\pi_{1}\left(B_{0, n} E^{2}\right)$, onde $E^{2}$ é o plano Euclideano. Este grupo clássico pode ser recuperado da definição acima como segue:

Tome um ponto base $\tilde{z^{0}}=\left(\tilde{z_{0}^{1}}, \ldots, \tilde{z_{0}^{n}}\right) \in F_{0, n} E^{2}$ para $\pi_{1}\left(F_{0, n} E^{2}\right)$ e um ponto base $z^{0} \in B_{0, n} E^{2}$ tal que a aplicação de recobrimento $p: F_{0, n} M \rightarrow B_{0, n} M$ satisfaz $p\left(\tilde{z^{0}}\right)=z^{0}$. 
Um elemento em $\pi_{1}\left(B_{0, n} E^{2}, z_{0}\right)$ é representado por um laço $f:(I,\{0,1\}) \rightarrow\left(B_{0, n} E^{2}, z_{0}\right)$ que se levanta unicamente a um caminho $\tilde{f}:(I,\{0\}) \rightarrow\left(F_{0, n} E^{2}, \tilde{z}^{0}\right)$.

Se $\tilde{f}(t)=\left(\tilde{f}_{1}(t), \ldots, \tilde{f}_{n}(t)\right) t \in I$, então cada função coordenada define via seu gráfico um arco $\alpha_{i}=\left\{\left(\tilde{f}_{i}(t), t\right) \in E^{2} \times I \mid t \in I\right\}$. Desde que $\tilde{f}(t) \in F_{0, n} E^{2}$ os $\operatorname{arcos} \alpha_{i}$ são disjuntos. A união $\Gamma=\alpha_{1} \cup \ldots \cup \alpha_{n}$ é chamada de trança geométrica, este por sua vez é representante de uma classe de caminhos do grupo fundamental $\pi_{1}\left(B_{0, n} E^{2}\right)$.

\subsection{Construção em dimensão um}

OBS: Usaremos $B_{0, n}$ para indicar $B_{0, n} E^{2}$ ou $B_{0, n}\left(\right.$ int $\left.D^{2}\right)$, e $F_{0, n}$ para indicar $F_{0, n} E^{2}$ ou $F_{0, n}\left(\right.$ int $\left.D^{2}\right)$.

Seja $K \subset S^{3}$, considere uma vizinhança tubular $V \cong K \times D^{2}$ de $K$ e um nó $L$ contido em int $V$ que seja o espaço total do recobrimento de $n$ folhas do nó $K$ considerando como projeção de recobrimento a restrição da projeção radial $p: V \rightarrow K$. O caso que nos interessa é quando $n$ é estritamente maior que zero, e quando $p \mid L: L \rightarrow K$ é difeomorfismo local.

Lema 18 Seja $L$ um nó contido no interior de uma vizinhança tubular $V$ de um nó $K$, de modo que considerando a restrição da projeção radial $p: V \rightarrow K$ a $L$, obtemos um difeomorfismo local que é um recobrimento de $n$ folhas de $K(L, p \mid, K), \quad n \in$ $\mathbb{N}-0$. Então existe aplicação $C^{\infty} \quad t: K \longrightarrow B_{0, n} E^{2}$ tal que $L=\{(x, p) \in K \times$ $\left(\right.$ int $\left.\left.D^{2}\right) \mid p \in t(x)\right\}$.

Dem. Nesta situação $L$ é o espaço total de um recobrimento de $n$ folhas de $K$ dado pela restrição da projeção radial de $V$ sobre $K$.

$$
\begin{gathered}
p: V \rightarrow K ; p(x, r, \theta)=(x, 0,0) \in K \times\{(0,0)\} \subset K \times D^{2} \cong V . \\
p \mid: L \rightarrow K \text { é recobrimento de } n \text { folhasedifeolocal.. }
\end{gathered}
$$

Aqui estamos parametrizando $D^{2}$ com coordenadas polares $(r, \theta)$.

Então para $x \in K$, existe vizinhança aberta $V_{x}$ conexa tal que

$$
(p \mid)^{-1}\left(V_{x}\right)=\bigcup_{i=1}^{n} W_{i}, W_{i} \cap W_{j}=\emptyset \quad(i \neq j)
$$




$$
p \mid W_{i}: W_{i} \rightarrow V_{x} \text { é um difeo local }
$$

com $W_{i}$ subconjunto aberto de $L$.

Definimos

$$
\begin{aligned}
& t: K \longrightarrow B_{0, n} E^{2}, \\
& t(x)=\left(\{x\} \times D^{2}\right) \cap L .
\end{aligned}
$$

Temos que $(p \mid)^{-1}\left(V_{x}\right)$ é união de abertos difeomorfos a $V_{x}$. Portanto pelo Teorema da Função Implícita temos que $W_{i}$ é igual ao gráfico de uma aplicação $C^{\infty}$ $t: V_{x} \rightarrow$ int $D^{2}$.

Assim temos uma aplicação $C^{\infty} \tilde{t}: V_{x} \rightarrow F_{0, n} E^{2}$, pois a topologia de $F_{0, n}$ é a topologia produto. E como a topologia de $B_{0, n}$ é a co-induzida pela projeção $C^{\infty}$ de $F_{0, n}$ sobre $B_{0, n}$, fica determinada a aplicação $C^{\infty}, t: K \rightarrow B_{0, n}$.

\subsection{Construção em dimensão dois}

Lema 19 Seja $M$ uma superfície orientável fechada contida em uma vizinhança tubular aberta $V$ de um mergulho $C^{\infty}$ de uma superfície orientável fechada conexa de genus $g, F_{g}^{2}$ em $S^{4}$, de modo que $M$ é o espaço total de um recobrimento de $n$ folhas de $F_{g}^{2}$ dado pela restrição da projeção radial de $V$ sobre $F_{g}^{2}$, restrição esta que é um difeo local. Então existe uma aplicação $C^{\infty} t: F_{g}^{2} \longrightarrow B_{0, n}$ tal que $M=\left\{(x, p) \in F_{g}^{2} \times\left(\right.\right.$ int $\left.\left.D^{2}\right) \mid p \in t(x)\right\}$.

Dem. Nesta situação

$$
p \mid: M \rightarrow F_{g}^{2} \text { é recobrimento de } n \text { folhas. }
$$

Então para $x \in F_{g}^{2}$, existe uma vizinhança aberta $V_{x}$ conexa tal que $p^{-1}\left(V_{x}\right)=$ $\bigcup_{i=1}^{n} W_{i}, \mathrm{com} W_{i} \cap W_{j}=\emptyset$ e com a restrição $p \mid W_{i}: W_{i} \longrightarrow V_{x}$ um difeo local, e mais, com $W_{i}$ subconjunto aberto de $M$. Temos como antes uma aplicação a múltiplos valores $r: F_{g}^{2} \rightarrow F_{g}^{2} \times\left(\right.$ int $\left.D^{2}\right) \cong V$ de modo que a imagem de $r$ é o satélite $M$.

Notemos também que para qualquer ponto $x \in F_{g}^{2}$ temos $n$ imagens $r(x)=\left\{t_{1}(x), t_{2}(x), \ldots, t_{n}(x)\right\}$; cada uma delas pertencente a $\{x\} \times\left(\right.$ int $\left.D^{2}\right) \mathrm{e}$ satisfazendo a condição $t_{i} \neq t_{j}$ se $i \neq j$. 
Como antes, a ordem na indexação das imagens $t_{1}(x), \ldots, t_{n}(x)$ não é relevante. Desse modo temos uma aplicação $C^{\infty}$ tomando valores em $F_{g}^{2}$ e com contradomínio $B_{0, n} E^{2}$.

$$
\begin{gathered}
t: F_{g}^{2} \longrightarrow B_{0, n} E^{2}, \\
t(x)=\left(x \times D^{2}\right) \cap M .
\end{gathered}
$$

\subsection{Construção em dimensão $k$}

Lema 20 Seja $M$ uma k-variedade orientável fechada contida em uma vizinhança tubular aberta $V$ de um mergulho $C^{\infty}$ de uma $k$-variedade orientável fechada conexa $F^{k}$ em $S^{k+2}$, de modo que $M$ é o espaço total de um recobrimento conexo de $n$ folhas de $F^{k}$ dado pela restrição da projeção radial de $V$ sobre $F^{k}, n \in \mathbb{N}-\{0\}$, restrição esta que é um difeo local. Então existe aplicação $C^{\infty} t: F^{k} \longrightarrow B_{0, n}$ tal que $M=\left\{(x, p) \in F^{k} \times\left(\right.\right.$ int $\left.\left.D^{2}\right) \mid p \in t(x)\right\}$.

OBS. Caso $\pi_{1} F^{k}=0$ a construção fornece apenas cópias paralelas de $F^{k}$.

\subsection{Definição da variedade satélite}

Dado um mergulho de uma $k$-variedade orientável fechada conexa $F^{k} \rightarrow S^{k+2}$ considere a aplicação de classe $C^{\infty}$.

$$
\begin{gathered}
h: F^{k} \rightarrow B_{0, n} \stackrel{\circ}{D^{2}}=\frac{F_{0, n} \stackrel{\circ}{D^{2}}}{\Sigma_{n}}=\frac{\left(\prod_{i=1}^{n} \stackrel{\circ}{D^{2}}\right)-\Delta}{\Sigma_{n}} \\
h(x)=\left[h_{1}(x), \ldots, h_{n}(x)\right]
\end{gathered}
$$

onde $\Delta$ é a diagonal gorda. () gráfico dessa aplicação pode ser traçado em $F^{k} \times D^{2}$ que é homeomorfa a vizinhança tubular aberta do mergulho de $F^{k}$ em $S^{k+2}$.

Na fibra $\stackrel{\circ}{D^{2}}$ solbre cada ponto $x \in F^{k}$ existem $n$ pontos $h_{1}(x), \ldots, h_{n}(x)$ de uma nova variedade formada pelos pontos da imagem de $h(x)$ desenhada na vizinhança tubular aberta $V$ de $F^{k}$. 
Veremos mais adiante que definindo apropriadamente a aplicação $h$ podemos ter um gráfico conexo.

Vamos fazer a seguinte convenção :

Chamemos a variedade obtida através de $h: F^{k} \rightarrow B_{0, n} E^{2}$ contida em $V \cong F^{k} \times \stackrel{\circ}{D^{2}}$ por $h F^{k}$. Estamos neste ponto fixando uma trivialização .

Temos assim uma variedade também de dimensão $k$ que é o espaço total de um recobrimento de $n$ folhas da variedade original $F^{k}$ mergulhada numa vizinhança tubular em $S^{k+2}$ de $F^{k}$, vindo daí a denominação de $h F^{k}$ ser uma variedade satélite de $F^{k}$.

A variedade $h F^{k}$ é bem determinada no seguinte sentido:

Lema 21 Duas variedades satélites de um mergulho $F^{k} \subset S^{k+2}$ obtidas através de aplicações homotópicas em $B_{0, n}$ são ambientemente isotópicas.

Dem. Suponha que existam duas aplicações $C^{\infty}$ :

$$
\begin{aligned}
& f: F^{k} \longrightarrow B_{0, n}, \\
& g: F^{k} \longrightarrow B_{0, n} .
\end{aligned}
$$

Suponha também que exista homotopia $C^{\infty}$ :

$$
\begin{gathered}
H: F^{k} \times I \longrightarrow B_{0, n}, \\
H(x, 0)=f(x), \text { e } H(x, 1)=g(x) .
\end{gathered}
$$

Considere a família de aplicações :

$$
\begin{gathered}
f_{t}: F^{k} \longrightarrow B_{0, n} \quad(t \in I) \\
f_{t}(x)=H(x, t), \quad(t \in I) .
\end{gathered}
$$

Vemos que $f_{t}\left(F^{k}\right) \subset F^{k} \times \operatorname{int} D^{2},(t \in I)$. Essa família é uma família $C^{\infty}$ que fornece uma isotopia

$$
\tilde{f}: F^{k} \times I \longrightarrow F^{K} \times i n t D^{2} .
$$

Usando o Teorema de Extensão de Isotopia conseguimos a isotopia ambiente

$$
J: F^{k} \times \operatorname{int} D^{2} \longrightarrow F^{k} \times \operatorname{int} D^{2}
$$




\subsection{Sobre Conexidade da Superfície Satélite}

Para o caso de superfícies $F_{g}^{2}$ podemos enunciar uma condição para que tenhamos a conexidade da superfície satélite.

Lema $22 B_{0, n}$ é um espaço $K(\pi, 1)$.

Dem. Vamos analisar inicialmente $F_{0,2}$.

$F_{0,2}$ se fibra sobre $E^{2}$ com fibra $E^{2}-\{1$ ponto $\}$, logo de

$$
\ldots \rightarrow \pi_{n}(\text { fibra }) \rightarrow \pi_{n}\left(F_{0,2}\right) \rightarrow \pi_{n}\left(E^{2}\right) \rightarrow \pi_{n-1}(\text { fibra }) \rightarrow \ldots
$$

obtemos

$$
\begin{gathered}
\pi_{n}\left(F_{0,2}\right)=0, \text { se } n \geq 2, \\
\pi_{1}\left(F_{0,2}\right) \cong \pi_{1}(\text { fibra }) \cong \mathbb{Z} .
\end{gathered}
$$

Observemos que $F_{0,3}$ se fibra sobre $F_{0,2}$ com fibra $E^{2}-\{2$ pontos $\}$, logo de

$$
\ldots \rightarrow \pi_{n}(\text { fibra } a) \pi_{n}\left(F_{0,3}\right) \rightarrow \pi_{n}\left(F_{0,2}\right) \rightarrow \pi_{n-1}(\text { fibra }) \rightarrow \ldots
$$

obtemos

$$
\pi_{n}\left(F_{0,3}\right)=0 \text { se } n \geq 2, .
$$

Prosseguindo a indução usando o fato de que $F_{0, s}$ se fibra sobre $F_{0, s-1} \mathrm{com}$ fibra $E^{2}-\{(s-1)$ pontos $\}$ conseguimos provar que $F_{0, n}$ é um espaço do tipo $K(\pi, 1)$.

Como $F_{0, n}$ é o espaço total do recobrimento de $B_{0, n}$ com fibra $\Sigma_{n}$ entendido como espaço topológico discreto concluimos que $\pi_{i}\left(B_{0, n}\right) \cong \pi_{i}\left(F_{0, n}\right), \quad i \geq 2$.

Portanto $B_{0, n}$ é espaço do tipo $K(\pi, 1)$.

Devemos recordar aqui o Teorema 8.1.11 de Spanier [35]:

"Seja $Y$ um espaço do tipo $K(\pi, 1)$. Para qualquer ( $\mathrm{W}$-complexo conexo $X$ o conjunto das classes de homotopia de aplicações de $X$ em $Y$ está em correspondência biunívoca com o conjunto das classes de homomorfismos entre $\pi_{1}\left(X, x_{0}\right)$ e $\pi_{1}\left(Y, y_{0}\right)$ " módulo classes de conjugação por elementos de $\pi_{1}\left(Y, y_{0}\right)$.

$$
[X, Y] \longleftrightarrow \operatorname{Hom}\left[\pi_{1}\left(X, x_{0}\right), \pi_{1}\left(Y, y_{0}\right)\right] / \sim
$$


Portanto para fornecer a menos de homotopia uma aplicação $h: F_{g}^{2} \rightarrow B_{0, n}$ é suficiente fornecer um homomorfismo entre $\pi_{1}\left(F_{g}^{2}\right)$ e $\pi_{1}\left(B_{0, n}\right)$.

Segundo Birman [5] $\pi_{1} B_{0, n}$ é finitamente apresentado por

$$
\pi_{1} B_{0, n}=\left\langle\sigma_{1}, \sigma_{2}, \ldots, \sigma_{n-1} ; \sigma_{i} \sigma_{j}=\sigma_{j} \sigma_{i} \text { se }|i-j| \geq 2, \sigma_{i} \sigma_{i+1} \sigma_{i}=\sigma_{i+1} \sigma_{i} \sigma_{i+1}\right.
$$

se $1 \leq i \leq(n-2)\rangle$. $h F_{r}^{2}$.

No caso de $h F_{r}^{2}$ ser conexa sabemos a relação entre o genus de $F_{g}^{2}$ e o genus de

Lema 23 Se $h F_{r}^{2}$ é variedade conexa então seu genus é $r=1-(1-g) . n$.

Dem. O genus de uma superfície conexa orientável fechada obedece a igualdade $\mathcal{X}=2-2 g$, onde $\mathcal{X}$ é a característica de Euler. Sabemos que $h F_{r}^{2}$ é um recobrimento de $n$ folhas de $F_{g}^{2}$. Prova-se também através de sequências espectrais que para uma fibração com base conexa por caminhos $B$, espaço total $E$ e fibra $J$, vale a igualdade $\mathcal{X}(E)=\mathcal{X}(J) \cdot \mathcal{X}(B)$.

No nosso caso de recobrimento a fibra é um conjunto discreto de $n$ pontos, logo $\mathcal{X}(F i b r a)=n$. Donde $\mathcal{X}\left(h F_{r}^{2}\right)=n \mathcal{X}\left(F_{g}^{2}\right)$ e portanto se chamarmos $r$ o genus de $h F_{r}^{2}$ obtemos

$$
2-2 r=(2-2 g) n \quad \text { implicando } \quad r=1-(1-g) n .
$$

Podemos também saber o genus de cada componente conexa de $h F_{r}^{2}$ caso saibamos o número de folhas do recobrimento que $h F_{r}^{2}$ restrita a esta componente faz sobre $F_{g}^{2}$.

A situação em geral é dada por um homomorfismo $h \pi: \pi_{1} F \rightarrow \pi_{1} B_{0, n}$.

Exemplo: Para obtermos $h: F_{g}^{2} \longrightarrow B_{0, n}$ constante, fazemos $h \pi$ o homomorfismo nulo. Neste caso o satélite terá $n$ componentes conexas, cada uma delas difeomorfa a $F_{g}^{2}$ original.

Considere o homomorfismo

$$
\begin{gathered}
\nu: \pi_{1} B_{0, n} \longrightarrow \Sigma_{n}, \\
\nu\left(\sigma_{i}\right)=\left(\begin{array}{ll}
i+1 \quad i
\end{array}\right) .
\end{gathered}
$$


Considere também a associação $\eta$ do grupo $\Sigma_{n}$ no conjunto finito $\mathbb{N}_{n}=\{1,2, \ldots, n\}$, que a cada permutação associa o número $\sigma(1)$, isto é:

$$
\begin{gathered}
\eta: \Sigma_{n} \longrightarrow \mathbb{N}_{n}, \\
\eta(\sigma)=\sigma(1) .
\end{gathered}
$$

Lema $24 A$ aplicação $\eta \circ \nu \circ h \pi$ é sobrejetora se e só se $h F_{r}^{2}$ tem apenas uma componente conexa.

Dem. Seja $x_{0}$ um ponto arbitrário de $F_{g}^{2}$, denotemos os $n$ pontos da intersecção da fibra sobre $x_{0}$ com o satélite $h F_{r}^{2},\left(x_{0} \times \stackrel{\circ}{D^{2}}\right) \cap h F_{r}^{2}$, por $z_{1}, z_{2}, \ldots, z_{n}$.

Suponhamos que $\eta \circ \nu \circ h \pi$ seja sobrejetora. Dados dois pontos $x_{1}$ e $x_{0}$ de $h F^{2}$ considere $y_{1}=p\left(x_{1}\right)$ e $y_{0}=p\left(x_{0}\right)$. Como $F^{2}$ é conexa existe caminho $\alpha$ ligando $y_{1}$ a $y_{0}$. Se $\beta$ é um levantamento de $\alpha$ que se inicia em $x_{1}$ então seu extremo final é um dos pontos $z_{i} \in\left(p \mid h F^{2}\right)^{-1}\left(y_{0}\right)$. Vamos assumir sem perda de generalidade que $x_{0}=z_{0}$. Como $\eta \circ \nu \circ h \pi$ é sobrejetora existe elemento $\gamma \in \pi_{1}\left(F^{2}, y_{0}\right)$ tal que $\eta \circ \nu \circ h \pi(\gamma)=i$ e portanto $h \pi(\gamma)$ é um caminho em $h F^{2}$ ligando $x_{0}=z_{0}$ a $z_{i}$. Logo o caminho justaposto $\beta * \gamma$ liga o ponto $x_{1}$ ao ponto $x_{0}$.

Suponhamos que $h F^{2}$ seja conexo. Então existe uma curva $\tilde{\beta}_{i}$ em $h F^{2}$ que passa pelos pontos $z_{1}$ e $z_{i}, i=1, \ldots, n$. A projeção $\beta=\left(p \mid h F^{2}\right)(\tilde{\beta})$ é uma curva $\gamma_{i} \in \pi_{1}\left(F^{2}, x_{0}\right)$ de modo que $\eta \circ \nu \circ h \pi\left(\gamma_{i}\right)=i, i=1, \ldots, n$.

OBS: Considerando a composta

$$
\nu \circ h \pi: \pi_{1} F_{g}^{2} \rightarrow \pi_{1} B_{0, n} \rightarrow \Sigma_{n}
$$

podemos definir a ação

$$
\begin{aligned}
& \phi: \pi_{1} F_{g}^{2} \times \mathbb{N}_{n} \rightarrow \mathbb{N}_{n} \\
& \phi(\gamma, i)=[\nu \circ h \pi(\gamma)](i) .
\end{aligned}
$$

Podemos enunciar o resultado:

"O número de componentes conexas do satélite é igual ao número de órbitas da ação $\phi . "$

Este resultado também vale no caso em que a dimensão da variedade $F$ é igual a $k$. 


\section{Existência da Variedade de Seifert}

Lema 25 Seja $V$ uma vizinhança tubular aberta de uma variedade orientável conexa $F^{k} \subset S^{k+2}$, na qual encontra-se a variedade $h F^{k}$ (não necessariamente conexa). Então existe uma variedade de Seifert para o satélite $h F^{k}$ que intercepta $S^{k+2}-V$ em $n$ cópias paralelas de uma variedade de Seifert de $F^{k}$.

OBS.1: Nesta seção não exigimos a condição de que a restrição ao satélite da projeção radial seja um recobrimento de $n$ folhas de $F^{k}$.

OBS.2: Caso $n$ seja igual a zero a variedade de Seifert para o satélite estará inteiramente contida em uma vizinhança tubular de $F^{k}$, nesse caso $\lambda: S^{1} \rightarrow S^{1}$ que aparece logo abaixo será tomada como aplicação constante.

OBS.3: A demonstração deste Lema será possível ao final da seção 6.4.

OBS.4: A partir deste ponto até a seção 6.4 .1 consideremos $h F^{k}$ variedade conexa.

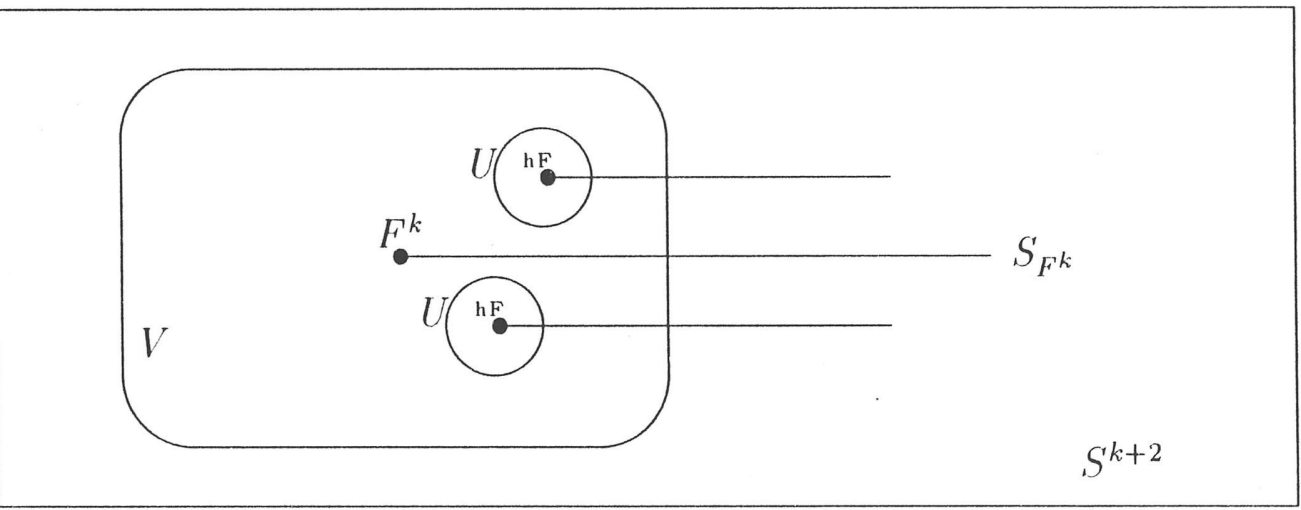

Seja $U$ vizinhança tubular aberta de $h F^{k}$ inteiramente contida em int $V$ e denote $W=\bar{V}-U$.

Existe uma aplicação diferenciável $f: X_{F^{k}}=S^{k+2}-V \rightarrow S^{1}$ tal que a variedade de Seifert $S_{F^{k}}$ de $F^{k}$ é obtida como imagem inversa de um valor regular $s_{o}$ de $S^{1}$, ver[27].

Podemos então obter $n$ cópias paralelas da variedade $S_{F^{k}}$ como imagem inversa de $n$ valores regulares existentes numa vizinhança em $S^{1}$ suficientemente pequena de $s_{0}$.

Nosso problema é conseguir uma aplicação $g: S^{k+2}-U \rightarrow S^{1}$ que coincida com $\lambda \circ f$ em $S^{k+2}-V$, onde $\lambda: S^{1} \rightarrow S^{1}$ é uma imersão $C^{\infty}$ de grau $n$. 
Podemos iniciar a definição de $g$ no espaço $\partial \bar{U}$ desde que seja fixada uma trivialização do fibrado normal de $h F^{k}$ que nos possibilite escrever o isomorfismo $\partial \bar{U} \cong h F^{k} \times S^{1}$.

Precisamos estudar a obstrução a extensão de aplicações de $(\partial \bar{U} \cup \partial \bar{V})=\partial W$ em $S^{1}$ para aplicações de $(W, \partial W)$ em $S^{1}$. E para podermos fazê-lo necessitamos antes estudar a trivialização dos fibrados normais $\nu_{F^{k}}$ de $F^{k}$ e $\nu_{h F^{k}}$ de $h F^{k}$ que nos dão os isomorfismos $\partial \bar{V} \cong F^{k} \times S^{1}$ e $\partial \bar{U} \cong h F^{k} \times S^{1}$.

\subsection{Trivialização de $\nu_{h F}$}

Lema 26 O fibrado normal $\nu_{h F^{k}}$ da variedade $h F^{k}$ é trivial.

A variedade $h F^{k}$ mergulha como subespaço fechado de $S^{k+2}$. Então por corolário 11.4 de [22] deduzimos que é nula a classe de Euler " $e\left(\nu_{h F^{k}}\right)$ " do fibrado normal de $h F^{k}$

Combinando este resultado com o Teorema 12.5 também de [22] obtemos a informação de que a segunda classe de obstrução para a existência de secção não nula definida no 2-esqueleto de $h F^{k}, o_{2}\left(\nu_{h F^{k}}\right) \in H^{2}\left(h F^{k}, \pi_{1}\left(S^{1}\right)\right)$, é uma classe nula.

Logo existe uma secção não nula $s: h F^{k} \longrightarrow \nu_{h F^{k}}$.

Conseguida uma secção não nula do fibrado $E^{2} \rightarrow \nu_{h F^{k}} \rightarrow h F^{k}$, como a dimensão da fibra é dois e o fibrado é orientável conseguimos uma outra secção linearmente independente com a primeira tomando sempre a direção ortogonal positiva.

('oncluimos portanto que $\nu_{h F^{k}}$ é um fibrado trivial.

Existem trivializações não equivalentes desse fibrado; A cada classe de homotopia de secções lincarmente independentes corresponde uma trivialização. Essas classes de equivalência estão em correspondência biunívoca com $H^{1}\left(h F^{k}\right)$ como segue.

Vamos supor que $H^{1}\left(h F^{k}\right) \cong \mathbb{Z}^{r}$.

Fixarla mona trivialização $T: U \rightarrow D^{2} \times h F^{k}$ fica definida uma classe $c\left[T^{\prime}\right] \in H^{1}\left(h F^{k}\right) \cong H o m\left(H_{1}\left(h F^{k}\right), Z Z\right)$ caracterizada da seguinte forma.

$$
\begin{gathered}
c[T]: H_{1}\left(h F^{k}\right) \longrightarrow \mathbb{L}, \\
c[T]([d])=L k_{S^{k+2}}\left(T(d), h F^{k}\right) .
\end{gathered}
$$


OBS. Chamamos a atenção para o fato de estarmos trabalhando com orientações fixadas para $S^{k+2}, F^{k}$ e $h F^{k}$, e para as variedades de Seifert tomamos as orientações compatíveis com seus bordos.

Aqui estamos usando a notação $T(d)$ para indicar a curva " $d$ " alçada a $S^{k+2}-h F^{k}$ através de $T$ ',ou melhor, através de uma fixada secção não nula das que determinam $T$.

Vamos usar o "número de intersecções " para calcular o "número de enlaçamento" acima.

Temos

$$
c[T]([d])=<T(d), S_{h F^{k}}>
$$

Vamos fixar uma base $B=\left\{\left[b_{1}\right], \ldots,\left[b_{r}\right]\right\}$ para $f\left[H_{1}\left(h F^{k}\right)\right]$.

Temos agora a seguinte identificação de $c[T]$ com uma $r$-upla:

$$
c[T]=\left(z_{1}, \ldots, z_{r}\right),
$$

onde

$$
z_{i}=<T\left(b_{i}\right), S_{h F^{k}}>
$$

Utilizando processo semelhante ao de [27] conseguimos uma trivialização distinguida $T_{0}$ que pode ser caracterizada pelo fato de $c\left[T_{0}\right]=(0, \ldots, 0)$.

Fixada una trivialização $T$ do fibrado normal $\nu_{h F^{k}}$ de $h F^{k}$ fica determinado (por restrição ao bordo) um homeomorfismo $\phi_{T}: \partial \bar{U} \rightarrow h F^{k} \times S^{1}$. Compondo-o com a projeção no segundo fator teremos

$$
f_{T}=\pi_{2} \circ \phi_{T}: \partial \bar{U} \rightarrow h F^{k} \times S^{1} \rightarrow S^{1} .
$$

Vemos portanto que para cada trivialização $T$, a aplicação $f_{T}$ leva a curva $T_{0}\left(b_{i}\right)$ em $z_{i}$-vezes a classe de orientação de $S^{1}$, isto é,

$$
\left[f_{T}\left(T_{0}\left(b_{i}\right)\right)\right]=z_{i} \cdot\left[S^{1}\right] .
$$

Denotemos o gerador de $H^{1}\left(S^{1}\right)$ por $\alpha$, onde $\alpha$ é identificado com o funcional

$$
\begin{gathered}
\alpha: H_{1}\left(S^{1}\right) \rightarrow \mathbb{Z}, \\
\alpha\left(\left[S^{1}\right]\right)=1 .
\end{gathered}
$$


Denotemos a classe fundamental de $S^{1}$ por $m$, e denotemos por $A$ a base $A=\{m\} \cup B=\left\{m,\left[b_{1}\right], \ldots,\left[b_{r}\right]\right\}$ para a homologia $f\left[H_{1}\left(S^{1}\right) \times H_{1}(h F)\right] \cong_{T_{0}} f\left[H_{1}(\partial \bar{U})\right]$.

Observemos que $f_{T}^{*}(\alpha) \in H^{1}(\partial \bar{U}) \cong T_{0} \mathbb{Z} \oplus \mathbb{Z}^{r}$ é identificado com a $(r+1)$-upla de inteiros $\left(1, z_{1}, \ldots, z_{r}\right)$ usando a base $A^{*}$ :

$$
\begin{gathered}
f_{T}^{*}(\alpha)\left(\left[T_{0}(m)\right]\right)=\alpha\left(\left[f_{T}\left(T_{0}(m)\right)\right]\right)=\alpha(m)=1, \\
f_{T}^{*}(\alpha)\left(T_{0}\left(b_{i}\right)\right)=\alpha\left(\left[f_{T}\left(T_{0}\left(b_{i}\right)\right)\right]\right)=\alpha\left(z_{i} \cdot m\right)=z_{i} \cdot \alpha(m)=z_{i}, 1 \leq i \leq r .
\end{gathered}
$$

Portanto

$$
f_{T}^{*}(\alpha)=m^{*}+z_{1} b_{1}^{*}+\cdots+z_{r} b_{r}^{*}
$$

\subsection{Trivialização de $\nu_{F^{k}}$}

Lema 27 O fibrado normal $\nu_{F^{k}}$ da variedade $F^{k}$ é trivial.

Dem. Similar a demonstração do lema da seção anterior.m

Denotando $S_{M}$ para a variedade de Seifert da variedade $M$, temos a existencia da aplicação $f: X_{F^{k}}=S^{k+2}-V \rightarrow S^{1}$ tal que $S_{F^{k}}=f^{-1}\left(s_{0}\right)$, onde $s_{0}$ é um valor regular de $f$.

Existe uma vizinhança $V_{0}$ em $S^{1}$ do ponto $s_{0}$, onde todos os pontos $x \in V_{0}$ são valores regulares da aplicação $f$.

Seja $\lambda: S^{1} \rightarrow S^{1}$ uma imersão $C^{\infty}$, de grau $n$, tal que pontos ordenados segundo orientação de $S^{1}\left\{s_{0}, s_{1}, \ldots, s_{n-1}\right\}$ de $V_{0}$ são levados em único ponto $r_{0} \in S^{1}$ e os

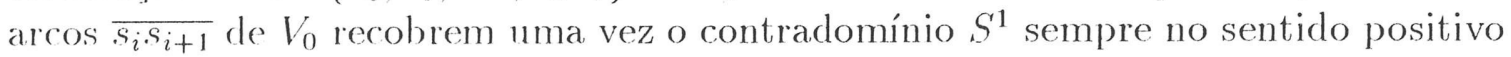
$\left(s_{n}=s_{0}\right)$.

() valor $r_{0}$ é um valor regular da composta $\lambda \circ f: X_{F^{k}} \rightarrow S^{1}$ e sua imagem inversa consiste de $n$ variedades de Seifert paralelas e difeomorfas.

Vamos supor que $f\left[H_{1}\left(F^{k}\right)\right] \cong Z^{s}$.

Vamos fixar uma base $D)=\left\{\left[a_{1}\right], \ldots,\left[a_{s}\right]\right\}$ para $f\left[H_{1}\left(F^{k}\right)\right]$ e utilizar a base dual $L^{*}=\left\{a_{1}^{*}, \ldots, a_{s}^{*}\right\}$ para $H^{1}\left(F^{k}\right)$. 
Como anteriormente cada trivialização $\Gamma: V \rightarrow F^{k} \times \stackrel{\circ}{D^{2}}$ da vizinhança tubular de $F^{k}$ determina uma classe $c[\Gamma] \in H^{1}\left(F^{k}\right)$, identificada com:

$$
\begin{gathered}
c[\Gamma]: H_{1}\left(F^{k}\right) \longrightarrow \mathbb{Z}, \\
c[\Gamma]([d])=L k_{S^{k+2}}\left(\Gamma(d), F^{k}\right)=<\Gamma(d), S_{F^{k}}>,
\end{gathered}
$$

onde $\Gamma(d)$ denota a curva " $d "$ alçada a $S^{k+2}-F^{k}$ através de $\Gamma$.

Temos a seguinte identificação :

$$
c[\Gamma]=\left(p_{1}, \ldots, p_{s}\right) \in \mathbb{Z}^{s},
$$

onde

$$
p_{i}=<\Gamma\left(a_{i}\right), S_{F^{k}}>.
$$

Existe uma trivialização distinguida $\Gamma_{0}$ que pode ser caracterizada pelo fato de $c\left[\Gamma_{0}\right]=(0, \ldots, 0)$.

Por restrição ao bordo, a trivialização fornece

$$
\phi_{\Gamma}: \partial \bar{V} \longrightarrow F^{k} \times S^{1},
$$

e por composição temos

$$
f_{\Gamma}=\lambda \circ \pi_{2} \circ \phi_{\Gamma}: \partial \bar{V} \rightarrow F^{k} \times S^{1} \rightarrow S^{1} \rightarrow S^{1}
$$

Vemos portanto que para cada trivialização $\Gamma$, a aplicação $f_{\Gamma}$ leva a curva $\Gamma_{0}\left(a_{i}\right)$ em n.p. $p_{i}$-vezes a classe de orientação de $S^{1}$, isto é,

$$
\left[f_{\Gamma}\left(\Gamma_{0}\left(a_{i}\right)\right)\right]=n \cdot p_{i} \cdot\left[S^{11}\right] .
$$

Vamos usar a base $E=\{m\} \cup D=\left\{m,\left[a_{1}\right], \ldots,\left[a_{s}\right]\right\}$ para a homologia $f\left[H_{1}(j \bar{V})\right] \cong_{\Gamma_{0}} f\left[H_{1}\left(S^{1}\right) \times H_{1}\left(F^{k}\right)\right]$ e usar a base dual $E^{*}=\left\{m^{*}, a_{1}^{*}, \ldots, a_{s}^{*}\right\}$ para a cohomologia $H^{1}(\partial \bar{V}) \cong \Gamma_{0} H^{1}\left(S^{1}\right) \times H^{1}\left(F^{k}\right)$.

Observemos que $f_{\Gamma}^{*} \in H^{1}(\partial \bar{V}) \cong \Gamma_{0} \mathbb{Z} \oplus \mathbb{Z}^{s}$ é identificado com a $(s+1)$-upla de inteiros $\left(n, n \cdot p_{1}, \ldots, n \cdot p_{s}\right)$. 


$$
\begin{gathered}
f_{\Gamma}^{*}(\alpha)\left(\Gamma_{0}(m)\right)=\alpha\left(f_{\Gamma}\left(\Gamma_{0}(m)\right)\right)=\alpha\left( \pm n \cdot\left[S^{1}\right]\right)= \pm n \\
f_{\Gamma}^{*}(\alpha)\left(\Gamma_{0}\left(a_{i}\right)\right)=\alpha\left(f_{\Gamma}\left(\Gamma_{0}\left(a_{i}\right)\right)\right)=\alpha\left(n . p_{i} \cdot\left[S^{1}\right]\right)=n \cdot p_{i}, 1 \leq i \leq s .
\end{gathered}
$$

Portanto $f_{\Gamma}^{*}(\alpha)=n \cdot m^{*}+n \cdot p_{1} a_{1}^{*}+\cdots+n \cdot p_{s} a_{s}^{*}$.

Percebemos nesse caso uma diferença essencial se o compararmos com o estudo da seção anterior.

Houve aqui a necessidade de utilizar uma aplicação $\lambda$ de grau $n$ em um dos fatores do produto cartesiano da trivialização do bordo da vizinhança tubular $V$. Essa utilização não permite o enunciado de um lema idêntico ao Lema 27.

Pois, enquanto o funcional

$$
f_{\Gamma}^{*}(\alpha): H_{1}\left(S^{1}\right) \times H_{1}\left(F^{k}\right) \cong_{\Gamma_{0}} H_{1}(\partial \bar{V}) \rightarrow \mathbb{Z}
$$

utiliza implicitamente a aplicação $\lambda: S^{1} \rightarrow S^{1}$, o funcional

$$
L k_{S^{k+2}}\left(\Gamma(-), F^{k}\right): H_{1}\left(S^{1}\right) \times H_{1}\left(F^{k}\right) \cong \Gamma_{\Gamma_{0}} H_{1}(\partial \bar{V}) \rightarrow \mathbb{Z}
$$

não tem a utilização da induzida em homologia da aplicação $\lambda: S^{1} \rightarrow S^{1}$.

Vamos considerar a aplicação

$$
\begin{gathered}
(\lambda, i d)_{*}: H_{1}\left(S^{1}\right) \times H_{1}(F) \longrightarrow H_{1}\left(S^{1}\right) \times H_{1}(F), \\
(\lambda, i d)_{*}([x],[y])=([\lambda(x)],[y]) .
\end{gathered}
$$

\subsection{Obstrução à extensão}

Vamos calcular $H^{1}(W)$. Vamos usar a notação $\bar{U}(-\epsilon)$ para indicar uma vizinhança tubular fechada inteiramente contida no interior da vizinhança tubular $U$ do satélite $h F^{k}$.

Vamos usar a notação $V_{(+\epsilon)}$ para indicar uma vizinhança tubular aberta que contém inteiramente a vizinhança tubular $\bar{V}$, isto é, $\bar{V} \subset V_{(+\epsilon)}$. 
Podemos assumir também que essas novas vizinhanças sejam obtidas através de uma variação suficientemente pequena das vizinhanças já consideradas $U$ e $V$, de modo que as inclusões acima sejam verificadas.

Considere $A=S^{k+2}-\bar{U}_{(-\epsilon)}$ e $B=V_{(+\epsilon)}$.

Nessas condições podemos aplicar o teorema de Mayer-Vietoris para a decomposição $S^{k+2}=A \cup B$, vendo que $A \cap B$ tem o mesmo tipo de homotopia de $W$, que $B$ tem o mesmo tipo de homotopia de $F^{k}$, e que $A$ tem o mesmo tipo de homotopia do complementar de uma vizinhança tubular do satélite $h F^{k}$.

$$
H^{1}(A \cup B) \stackrel{j_{1}^{*}}{\longrightarrow} H^{1}(A) \oplus H^{1}(B) \stackrel{i_{1}^{*}}{\longrightarrow} H^{1}(A \cap B) \stackrel{\delta_{1}^{*}}{\longrightarrow} H^{2}(A \cup B)
$$

torna-se

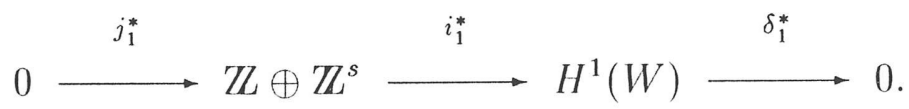

Estamos aqui considerando como gerador de $H_{1}(A)$ o meridiano de $h F^{k}$ que denotaremos por $m h_{h k}$, e para isso estamos usando a trivialização $T_{0}$ da vizinlanuça tubular $U$.

Estamos também considerando como geradores de $H_{1}(B)$ os elementos da base $D$ e para isso estamos usando a trivialização $\Gamma_{0}$ da vizinhança tubular $V$, e denotaremos o meridiano de $F^{k}$ por $m_{F^{k}}$.

Sabemos portanto que

$$
H^{1}(W) \cong H^{1}\left(S^{k+2}-U\right) \oplus H^{1}\left(F^{k} \times D^{2}\right) \cong T_{0} \cup \Gamma_{0} \mathbb{Z} \oplus \mathbb{Z}^{s} .
$$

I) (evemos analisar agora a sequência exata do par $(W, \partial W)$ e o morfismo $f_{\Gamma}^{*} \uplus f_{T}^{*}$.

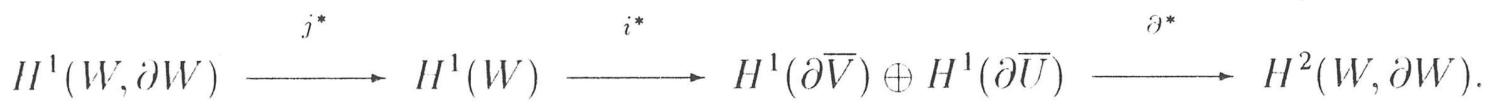

$$
H^{1}\left(S^{1}\right) \longrightarrow f_{\Gamma}^{*} \oplus f_{T}^{*} \longrightarrow H^{1}(\partial \bar{V}) \oplus H^{1}(\partial \bar{U}) .
$$

Lema $28 S_{\mathrm{C}}\left(\int_{\Gamma}^{*} \oplus \int_{T}^{*}\right)(\alpha) \in \mathrm{Ker} \partial^{*}$, então existe uma extensão de $\left(f_{\Gamma} \cup f_{T}\right): \partial W=\partial V \cup \partial U \rightarrow S^{1}$ para $g:(W, \partial W) \rightarrow S^{1}$. 
Dem. Temos uma aplicação $\left(f_{\Gamma} \cup f_{T}\right): \partial W=\partial \bar{V} \cup \partial \bar{U} \rightarrow S^{1}$ a qual queremos estender para todo $W$ que é de dimensão $k+2$.

A extensão para o 0-esqueleto sempre existe e como $W$ é conexo por caminhos também existe extensão para o 1-esqueleto de $(W, \partial W)$. Como as obstruções a extensão são

$$
\left[c^{n}\left(f_{\Gamma} \cup f_{T}\right)\right] \in H^{n}\left(W, \partial W ; \pi_{n-1}\left(S^{1}\right)\right),
$$

o único cociclo de obstrução possivelmente não nulo é:

$$
\begin{gathered}
c^{2}\left(f_{\Gamma} \cup f_{T}\right) \in C^{2}(W, \partial W) \cong H o m\left(C_{2}(W, \partial W), \mathbb{Z}\right), \\
{\left[c^{2}\left(f_{\Gamma} \cup f_{T}\right)\right] \in H^{2}(W, \partial W ; \mathbb{Z}) .}
\end{gathered}
$$

Este último é dado por: Se $[\sigma] \in H_{2}(W, \partial W)$ então

$$
\left[c^{2}\left(f_{\Gamma} \cup f_{T}\right)\right][\sigma]=\left[\left(f_{\Gamma} \cup f_{T}\right)(\partial \sigma)\right]
$$

Lembremos que

$$
\begin{gathered}
H^{1}(\partial W) \cong \operatorname{Hom}\left(H_{1}(\partial W), \mathbb{Z}\right) \mathrm{e} \\
H^{1}\left(S^{1}\right) \cong H o m\left(H_{1}\left(S^{1}\right), \mathbb{Z}\right) .
\end{gathered}
$$

Onde $\alpha$, o gerador de $H^{1}\left(S^{1}\right)$, é caracterizado por $\alpha=\left[S^{1}\right]^{*}$ tal que $\left[S^{1}\right]^{*}\left(\left[S^{1}\right]\right)=$ 1 .

$$
f_{\Gamma}^{*} \oplus f_{T}^{*}: H^{1}\left(S^{1}\right) \rightarrow H^{1}(\partial W)
$$

satisfaz:

$$
\begin{gathered}
\left(f_{\Gamma}^{*} \oplus f_{T}^{*}\right)(\alpha): H_{1}(\partial W) \rightarrow \mathbb{Z} \text { é dada por } \\
\left(f_{\Gamma}^{*} \oplus \int_{T}^{*}\right)(\alpha)[\beta]=\alpha\left(\left[\left(f_{\Gamma} \cup f_{T}\right)(\beta)\right]\right),
\end{gathered}
$$




$$
\begin{gathered}
\partial^{*}\left(f_{\Gamma}^{*} \oplus f_{T}^{*}\right)(\alpha) \in \operatorname{Hom}\left(H_{2}(W, \partial W), \mathbb{Z}\right) \text { é dada por } \\
\partial^{*}\left(f_{\Gamma}^{*} \oplus f_{T}^{*}\right)(\alpha)[\sigma]=\left(f_{\Gamma}^{*} \oplus f_{T}^{*}\right)(\alpha)[\partial(\sigma)]=\alpha\left(\left[\left(f_{\Gamma} \cup f_{T}\right)(\partial \sigma)\right]\right) .
\end{gathered}
$$

Então se $\left(f_{\Gamma}^{*} \oplus f_{T}^{*}\right)(\alpha) \in K \operatorname{Ker} \partial^{*}$ teremos:

$$
\partial^{*}\left(f_{\Gamma}^{*} \oplus f_{T}^{*}\right)(\alpha)[\sigma]=0=\alpha\left(\left[\left(f_{\Gamma} \cup f_{T}\right)(\partial \sigma)\right]\right) \operatorname{com} \alpha \neq 0 .
$$

Logo $\left[\left(f_{\Gamma} \cup f_{T}\right)(\partial \sigma)\right]=0$ e portanto $\left[c^{2}\left(f_{\Gamma} \cup f_{T}\right)\right][\sigma]=0$.

Ou seja, é possível estender $f_{\Gamma} \cup f_{T}$ ao $(k+2)$-esqueleto de $(W, \partial W)$. Como a sequência de cohomologia do par $(W, \partial W)$ é exata, se $\left(\int_{\Gamma}^{*} \oplus f_{T}^{*}\right)(\alpha) \in \operatorname{Im} i^{*}$ também existe extensão.

Se $\left(f_{\Gamma}^{*} \oplus f_{T}^{*}\right)(\alpha) \in \operatorname{Im} i^{*}$ existe uma aplicação $g: W \rightarrow S^{1}$ que estende $f_{\Gamma} \cup f_{T}$ : $\partial W \rightarrow S^{1}$, e poderemos então tomar um valor regular $s_{0} \in S^{1}$ e tomar $g^{-1}\left(x_{0}\right)$ que será uma variedade $(k+1)$-dimensional de Seifert em $W$ que será "colada" com as $n$ cópias da Seifert de $F^{k}$ existentes no exterior da vizinhança tubular $V$.

\subsection{Existência da extensão}

Consideremos as inclusões: $i_{V}: V \rightarrow W$ e $i_{U}: U \rightarrow W$.

Devemos estudar o homomorfismo $i^{*}=i_{V}^{*}+i_{U}^{*}=\left(i_{V}^{*}, i_{U}^{*}\right)$.

$$
\mathbb{Z} \oplus \mathbb{Z}^{s} \cong_{T_{0} \cup \Gamma_{0}} H^{1}(W) \stackrel{i^{*}}{\longrightarrow} H^{1}(\partial V) \oplus H^{1}(\partial U) \cong_{\Gamma_{0} \cup T_{0}}\left(\mathbb{Z} \oplus \mathbb{Z}^{s}\right) \oplus\left(\mathbb{Z} \oplus \mathbb{Z}^{r}\right) .
$$

Usando a base $D$, os geradores do domínio de $i^{*}$ são $\left\{m_{h F^{*}}^{*},\left[a_{1}, \ldots,\left[a_{s}\right]\right\}\right.$.

- (a) $m_{h F^{k}}^{*} \in H^{1}\left(S^{k+2}-U\right) \cong \operatorname{Hom}\left(H_{1}\left(S^{k+2}-U\right), \mathbb{Z}\right)$ dado por $m_{h F^{*}}^{*}\left(\left[m_{h F^{k}}\right]\right)=1$ e $m_{h F^{k}}^{*}\left(\left[a_{i}\right]\right)=0, \forall 1 \leq i \leq s$. 
- (b) $a_{i}^{*} \in H^{1}\left(F^{k} \times D^{2}\right) \cong H \operatorname{Hom}\left(H_{1}\left(F^{k}\right) ; \mathbb{Z}\right)$ dado por $a_{i}^{*}\left(\left[a_{j}\right]\right)=\delta_{i j}$, $\forall 1 \leq i, j \leq s$ e $a_{i}^{*}\left(\left[m_{h F^{k}}\right]\right)=0, \forall 1 \leq i \leq s$.

Os geradores do contradomínio de $i^{*}$ são:

- $\operatorname{Em} H^{1}(\partial \bar{V}) \cong \operatorname{Hom}\left(H_{1}(\partial \bar{V}), \mathbb{Z}\right) \cong \Gamma_{0} \mathbb{Z} \oplus \mathbb{Z}^{s}$ vamos usar a base dual $E^{*}$. A imagem de $m_{h F^{k}}^{*}$ por $i_{V}^{*}$ é

$$
i_{V}^{*} m_{h F^{k}}^{*}: H_{1}(\partial \bar{V}) \cong_{\Gamma_{0}} H_{1}\left(S^{1}\right) \oplus H_{1}\left(F^{k}\right) \rightarrow \mathbb{Z} \text { caracterizada por }
$$

$i_{V}^{*} m_{h F^{k}}^{*}\left(\left[a_{i}\right]\right)=m_{h F^{k}}^{*}\left(i_{V *}\left(a_{i}\right)\right)=m_{h F^{k}}^{*}\left(\left[a_{i}\right]\right)=0$,

$i_{V}^{*} m_{h F^{k}}^{*}([m])=m_{h F^{k}}^{*}\left(i_{V *}[m]\right)=m_{h F^{k}}^{*}\left(n \cdot\left[m_{h F^{k}}\right]\right)=n$.

As imagens dos geradores $a_{i}^{*}$ por $i_{V}^{*}$ são

$$
i_{V}^{*} a_{i}^{*}: H_{1}(\partial \bar{V}) \cong_{\Gamma_{0}} H_{1}\left(S^{1}\right) \oplus H_{1}\left(F^{k}\right) \rightarrow \mathbb{Z} \text { caracterizada por }
$$

$i_{V}^{*} a_{i}^{*}([m])=a_{i}^{*}\left(i_{V *}[m]\right)=a_{i}^{*}\left(n\left[m_{h F^{k}}\right]\right)=0$,

$i_{V}^{*} a_{i}^{*}\left(\left[a_{j}\right]\right)=a_{i}^{*}\left(i_{V *}\left[a_{j}\right]\right) 0=a_{i}^{*}\left(\left[a_{j}\right]\right)=\delta_{i j}$.

Portanto vemos que

$$
H^{1}\left(S^{1}\right) \oplus H^{1}\left(F^{k}\right) \cong T_{0} \cup \Gamma_{0} H^{1}(W) \longrightarrow i_{V}^{*} \longrightarrow H^{1}(\partial \bar{V}) \cong \Gamma_{0} H^{1}\left(S^{1}\right) \oplus H^{1}\left(F^{k}\right)
$$

é sobrejetora no conjunto dos vetores de $\mathbb{Z} \oplus \mathbb{Z}^{s} \cong_{\Gamma_{0}} H^{1}(\partial \bar{V})$ que são da forma (n.k,s-upla), $k \in \mathbb{Z}$.

- $\operatorname{Em} H^{1}(\partial \bar{U}) \cong \operatorname{Hom}\left(H_{1}(\partial \bar{U}), \mathbb{Z}\right) \cong T_{0} \mathbb{Z} \oplus \mathbb{Z}^{r}$ vamos usar a base dual $A^{*}$.

A imagem de $m_{h F^{k}}^{*}$ por $i_{U}^{*}$ é

$i_{I}^{*} m_{h F^{k}}^{*}: H_{1}(\partial \bar{U}) \cong T_{0} H_{1}\left(S^{1}\right) \oplus H_{1}\left(h F^{k}\right) \rightarrow Z Z$ caracterizada por

$i_{U}^{*} m_{h F^{k}}^{*}\left(\left[b_{i}\right]\right)=m_{h F^{k}}^{*}\left(i_{U *}\left[b_{i}\right]\right)=m_{h F^{k}}^{*}\left(\left[b_{i}\right]\right)=0$,

$i_{l}^{*} m_{h F^{k}}^{*}([m])=m_{h F^{k}}^{*}\left(i_{U *}[m]\right)=m_{h F^{k}}^{*}\left(\left[m_{h F^{k}}\right]\right)=1$.

A imagem dos geradores $a_{i}^{*}$ por $i_{U}^{*}$ é

$$
i_{U}^{*} a_{i}^{*}: H_{1}(\partial \bar{U}) \cong_{T_{0}} H_{1}\left(S^{1}\right) \oplus H_{1}\left(h F^{k}\right) \rightarrow \mathbb{Z} \text { caracterizado por }
$$


$i_{U}^{*} a_{i}^{*}([m])=a_{i}^{*}\left(i_{U *}[m]\right)=a_{i}^{*}\left[i_{U}(m)\right]=a_{i}^{*}\left[m_{h F^{k}}\right]=0$.

Então a imagem do gerador $a_{i}^{*}$ pela aplicação $i_{U}^{*}$ é combinação linear finita apenas dos geradores $b_{i}^{*}$ do contradomínio $H^{1}\left(S^{1}\right) \oplus H^{1}\left(h F^{k}\right) \cong_{\Gamma_{0}} H^{1}(\partial \bar{U})$.

Demonstração do Lema 25:

Utilizamos a trivialização $\Gamma_{0}: V \rightarrow F^{k} \times\left(\right.$ int $\left.D^{2}\right)$ para construir as cópias paralelas da variedade de Seifert para $F^{k}$ em $S^{k+2}-V$ e deste modo pelos cálculos da seção 6.2 temos $f_{\Gamma_{0}}^{*}(\alpha)=(n, 0, \ldots, 0)$.

$O$ vetor $(n, 0, \ldots, 0)$ acima pertence a $\operatorname{Im}\left(i_{V}^{*}\right)$, e mais, podemos ver claramente que só o elemento

$$
m_{h F^{k}}^{*}+0 . a_{1}^{*}+0 . a_{2}^{*}+\ldots+0 . a_{s}^{*} \in H^{1}(W)
$$

é tal que

$$
i_{V}^{*}\left(m_{h F^{k}}^{*}+0 . a_{1}^{*}+\ldots+0 . a_{s}^{*}\right)=(n, 0, \ldots, 0) .
$$

Vemos agora que

$$
i_{U}^{*}\left(m_{h F^{k}}^{*}+0 . a_{1}^{*}+\ldots+0 . a_{s}^{*}\right)=(1,0, \ldots, 0) .
$$

Devemos portanto tomar a trivialização distinguida $T_{0}: \partial \bar{U} \rightarrow S^{1} \times h F^{k}$ para termos

$$
\begin{gathered}
\left(f_{\Gamma_{0}}^{*} \oplus f_{T_{0}}^{*}\right)(\alpha)=(n, 0, \ldots, 0) \oplus(1,0, \ldots, 0)= \\
\left(i_{V}^{*} \oplus i_{U}^{*}\right)\left(m_{h F^{k}}^{*}+0 . a_{1}^{*}+\ldots+0 . a_{s}^{*}\right) .
\end{gathered}
$$

()BS: Chamamos então a atenção ao fato de que para conseguir uma aplicação $g:(W, \partial W) \rightarrow S^{1}$, extensão de $\left(h_{1} \cup h_{2}\right): \partial \bar{U} \cup \partial \bar{V} \rightarrow S^{1}$, a definição da aplicação $h_{1}: \partial \bar{U} \rightarrow S^{1}$ fica determinada pela escolha,ou melhor, pela definição da aplicação $h_{2}: \partial \bar{V} \rightarrow S^{1}$.

Esta demonstrado o Lema 25.

\subsubsection{Modificações para $h F^{k}$ desconexa}

Devemos observar que a variedade $h F^{k}$ pode ter várias componentes conexas e mesmo assim o Lema 25 continua valendo. 
Suponhamos que $h F^{k}$ seja formado por $p$ componentes conexas

$$
h F^{k}=h F_{1}^{k} \cup h F_{2}^{k} \cup \ldots \cup h F_{p}^{k}
$$

Denotemos por $U_{j}$ a vizinhança tubular da componente $j$, e por $U$ a união dessas vizinhanças.

O Lema 26 pode ser aplicado a cada componente conexa e sua demonstração continua inalterada. Concluimos assim que o fibrado normal de cada $h F_{i}^{k}$ é trivial.

Precisaremos fixar uma base

$$
B^{i}=\left\{b_{1}^{i}, b_{2}^{i}, \ldots, b_{r(i)}^{i}\right\}
$$

para cada homologia $H_{1}\left(h F_{i}^{k}\right)$.

Uma trivialização do fibrado normal da variedade satélite é uma união de trivializações, uma para cada componente, isto é;

$$
T: U \rightarrow h F^{k} \times\left(\text { int } D^{2}\right)
$$

é uma união

$$
T=\cup_{i=1}^{p} T^{i}, \quad T^{i}: U_{i} \rightarrow h F_{i}^{k} \times\left(\operatorname{int} D^{2}\right) .
$$

Como antes temos as classes $c\left[T^{i}\right] \in H^{1}\left(h F_{i}^{k}\right)$ caracterizadas da seguinte forma:

$$
\begin{gathered}
c\left[T^{i}\right]: H^{1}\left(h F_{i}^{k}\right) \rightarrow \mathbb{Z}, \\
c\left[T^{i}\right]([d])=<T^{i}(d), S_{h F^{k}}>.
\end{gathered}
$$

Usando as bases $B^{i}$ identificamos as classes acima com as $r(i)$-uplas

$$
c\left[T^{i}\right]=\left(z_{1}^{i}, z_{2}^{i}, \ldots, z_{r(i)}^{i}\right),
$$

onde

$$
z_{j}^{i}=<T^{i}\left(b_{j}\right), S_{h F^{k}}>
$$

Existe uma trivialização distinguida $T_{0}$, obtida a partir da variedade de Seifert global do mergulho h. $F^{k}$ como faz [27].

Fixada uma trivialização $T=\cup_{i=1}^{p} T^{i}$, construimos uma aplicação 


$$
f_{T}^{*}: H^{1}\left(S^{1}\right) \longrightarrow H^{1}(\partial \bar{U}) \cong T_{0} \bigoplus_{i=1}^{p}\left[\mathbb{Z} \oplus \mathbb{Z}^{r(i)}\right]
$$

Podemos portanto escrever

$$
f_{T}^{*}(\alpha)\left(1, z_{1}^{1}, \ldots, z_{r(1)}^{1}, 1, z_{1}^{2}, \ldots, z_{r(2)}^{2}, \ldots, 1, z_{1}^{p}, \ldots, z_{r(p)}^{p}\right) .
$$

Ao estudarmos o homomorfismo $i^{*}=\left(i_{V}^{*}, i_{U}^{*}\right)$ existirão algumas modificações .

O homomorfismo em questão é:

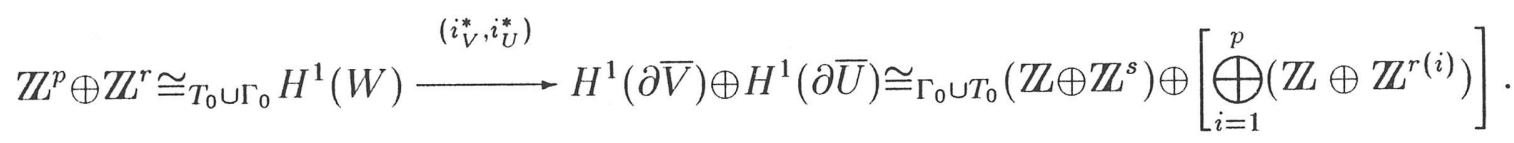

Denotando por $m_{h F_{i}^{k}}$ os meridianos das componentes $h F_{i}^{k}$, podemos refazer os cálculos da seção 6.4 e chegar a conclusão de que partindo da trivialização $\Gamma_{0}: V \rightarrow F^{k} \times\left(\right.$ int $\left.D^{2}\right)$ para construir as cópias paralelas da variedade de Seifert para $F^{k}$ em $S^{k+2}-V$, devemos tomar a trivialização distinguida $T_{0}=\cup_{i=1}^{p} T^{i}$ para que fique verdadeira a inclusão

$$
\left(f_{\Gamma_{0}}^{*} \oplus f_{T_{0}}^{*}\right)(\alpha) \in \operatorname{Im}\left(i_{V}^{*}, i_{U}^{*}\right) .
$$




\section{Estudo dos Módulos de Alexander}

\subsection{Decomposição}

Tomemos um mergulho de uma variedade fechada orientável conexa $F^{k} \rightarrow S^{k+2}$ e seja $M$ (orientada e possivelmente desconexa) a variedade obtida através da aplicação $h: F^{k} \rightarrow B_{0, n} E^{2}$.

Seja $V=F^{k} \times \stackrel{\circ}{D^{2}} \subset S^{k+2}$.

Então temos $M$ homologicamente equivalente a $n$ vezes a variedade original $F^{k}$ em $V, M \sim n . F^{k}$.

Seja $U$ vizinhança tubular $U=M \times \stackrel{\circ}{D^{2}}$, contida em $V$.

Vamos usar as seguintes notações :

$$
X=S^{k+2}-U ; \quad W=\bar{V}-U ; \quad Y=S^{k+2}-V ; \quad T=\partial V .
$$

Consideremos as inclusões

$$
i_{1}: W \rightarrow X ; \quad i_{2}: Y \rightarrow X ; \quad i_{3}: T \rightarrow X .
$$

Tome um ponto base $x \in T$.

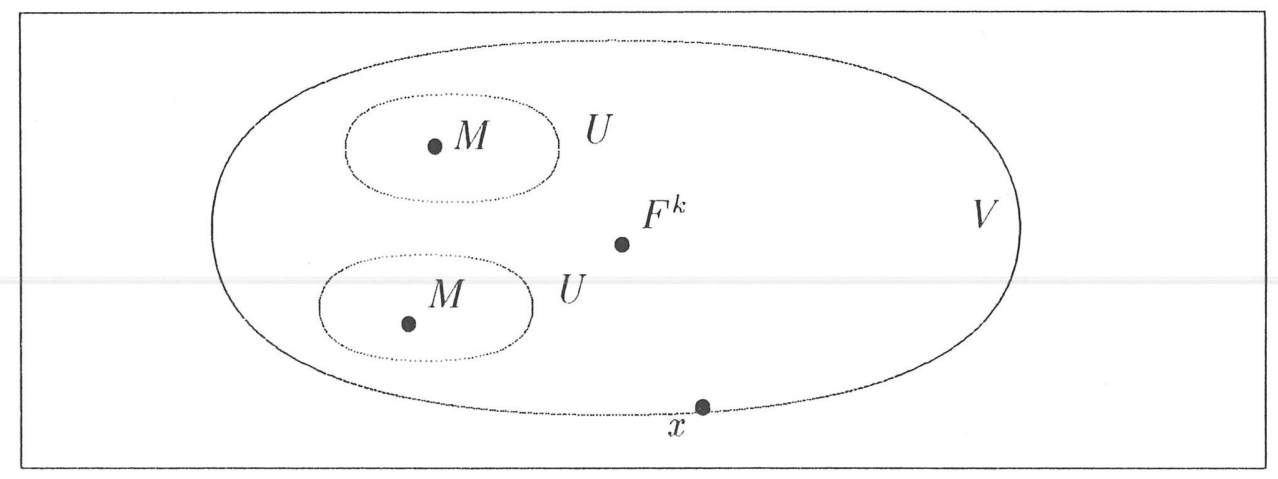

Seja 


$$
\begin{gathered}
\phi: \pi_{1}(X, x) \rightarrow \mathbb{Z} \cong G=<t,> \\
\phi(g)=t^{L k(g, M)} ; g \in \pi_{1}(X, x) \text { onde } L k(g, M)=<g, S_{M}>
\end{gathered}
$$

E seja $p: \tilde{X} \rightarrow X$ o recobrimento cíclico infinito associado a $\operatorname{Ker} \phi$. Denote $\tilde{W}=p^{-1}(W), \quad \tilde{Y}=p^{-1}(Y), \quad \tilde{T}=p^{-1}(T)$.

Então vale $\tilde{X}=\tilde{W} \cup \tilde{Y}$ e $\tilde{W} \cap \tilde{Y}=\tilde{T}$.

Vendo que para $\forall g \in \pi_{1}(Y, x)$

$$
\begin{gathered}
L k(g, M)=<g, S_{M}>=<g, n . S_{F^{k}}>= \\
=n .<g, S_{F^{k}}>=n . L k\left(g, F^{k}\right),
\end{gathered}
$$

temos a composta:

$$
\begin{gathered}
\pi_{1}(Y, x) \stackrel{i_{2 *}}{\longrightarrow} \pi_{1}(X, x) \stackrel{\phi}{\longrightarrow} G, \\
\left(\phi \circ i_{2 *}\right)(g)=t^{n \cdot L k\left(g, F^{k}\right)},
\end{gathered}
$$

ou seja $\phi \circ i_{2 *}$ leva $\pi_{1}(Y, x)$ sobre o subgrupo $H=<t^{n},>\operatorname{de} G$.

A partir de agora quando nos referirmos ao espaço total do recobrimento cíclico infinito do complementar da variedade $Q$ usaremos a notação $\tilde{X}_{Q}$.

Seja $Y_{1}$ uma das $n$ componentes conexas de $\tilde{Y}$. Então $p \mid Y_{1}: Y_{1} \rightarrow Y$ é um recobrimento cíclico infinito associado ao $\operatorname{Ker}\left(\phi \circ i_{2 *}\right)$. $Y$ é o complemento de uma vizinhança tubular de $F^{k}$ em $S^{k+2}$ e então $H_{q}\left(Y_{1}\right) \cong H_{q}\left(\tilde{X}_{F^{k}}\right)$.

Temos a composta:

$$
\begin{gathered}
\pi_{1}(W, x) \stackrel{i_{1 *}}{\longrightarrow} \pi_{1}(X, x) \stackrel{\phi}{\longrightarrow} G, \\
\left(\phi \circ i_{1 *}\right)(g)=t^{L k(g, M)},
\end{gathered}
$$

e vemos que $p \mid \tilde{W}: \tilde{W} \rightarrow W$ é um recobrimento cíclico infinito associado a $\operatorname{lier}\left(\phi \circ i_{1 *}\right)$.

Temos a composta: 


$$
\begin{gathered}
\pi_{1}(T, x) \stackrel{i_{3 *}}{\longrightarrow} \pi_{1}(X, x) \stackrel{\phi}{\longrightarrow} G, \\
\left(\phi \circ i_{3 *}\right)(g)=t^{L k(g, M)},
\end{gathered}
$$

mas como para $\forall g \in \pi_{1}(T, x)$ temos

$$
<g, S_{M}>=n .<g, S_{F^{k}}>,
$$

então a composta acima fica

$$
\left(\phi \circ i_{3 *}\right)(g)=t^{n \cdot L k\left(g, F^{k}\right)} .
$$

Isto é $\phi \circ i_{3 *}$ leva $\pi_{1}(T, x)$ sobre o subgrupo $H=<t^{n},>$ de $G$.

Denotemos por $T_{i}$ a $i$-ésima componente conexa de $\tilde{T}$, então $T_{i}$ é homeomorfa a $F^{k} \times \mathbb{R}$.

Denotemos por $Y_{i}$ a $i$-ésima componente de $\tilde{Y}$.

No espaço $\tilde{X}$ vamos escolher um "nível" chamando-o de $\tilde{X}_{0}$, o qual é homeomorfo a $X$ menos uma vizinhança bicolar da variedade de Seifert de $F^{k}$.

Das $n$ componentes $T_{i}$ de $\tilde{T}$ que interceptam $\tilde{X}_{0}$, escolhemos uma e denotamos por $T_{0}$. Denotamos também por $Y_{0}$ dentre as $n$ componentes de $\tilde{Y}$, aquela que tem $T_{0}$ como bordo.

Através da ação dos elementos $t^{i} \in \Lambda, i \in \mathbb{Z}$, vamos indexando subespaços de $\tilde{X}$, chamando de "nível" $\tilde{X}_{i}$ a imagem $t^{i}\left(\tilde{X}_{0}\right)$.

Fazemos também as "indexações"

$$
\begin{aligned}
& T_{i}=t^{i}\left(T_{0}\right), i=1, \ldots n-1 \mathrm{e} \\
& Y_{i}=t^{i}\left(Y_{0}\right), i=1, \ldots, n-1 .
\end{aligned}
$$

Deste modo temos compatibilidade de notações entre as estruturas de $\Lambda_{n}$-módulos de $H_{*}\left(T_{i}\right)$ e $H_{*}\left(Y_{i}\right)$ com as estruturas de $\Lambda$-módulos de

$$
H_{*}(\tilde{T})=\bigoplus_{i=0}^{n-1} H_{*}\left(T_{i}\right) \text { e } H_{*}(\tilde{Y})=\bigoplus_{i=0}^{n-1} H_{*}\left(Y_{i}\right) \text {. }
$$

A $\Lambda$-estrutura de $H_{*}(\tilde{T})$ é dada pela extensão linear da ação de $t \in \Lambda$ definida por:

$$
t: H_{*}(\tilde{T}) \longrightarrow H_{*}(\tilde{T}),
$$




$$
t\left(a_{0}, a_{1}, \ldots, a_{n-1}\right)=\left(t^{n} a_{n-1}, a_{0}, a_{1}, \ldots, a_{n-2}\right) .
$$

A $\Lambda$-estrutura de $H_{*}(\tilde{Y})$ é dada pela extensão linear da ação de $t \in \Lambda$ definida por:

$$
\begin{gathered}
t: H_{*}(\tilde{Y}) \longrightarrow H_{*}(\tilde{Y}) \\
t\left(b_{0}, b_{1}, \ldots, b_{n-1}\right)=\left(t^{n} b_{n-1}, b_{0}, b_{1}, \ldots, b_{n-2}\right) .
\end{gathered}
$$

Devemos chamar a atenção para o fato de que argumentos similares aos do Lema 11 provam que $H_{i}\left(\tilde{X}_{M}\right) \cong 0, i>k$.

Podemos agora usar a sequência de Mayer-Vietoris para a decomposição $\tilde{X}_{M}=$ $\tilde{W} \cup \tilde{Y}$.

Usaremos as seguintes inclusões:

$$
\begin{gathered}
i: \tilde{T} \rightarrow \tilde{W}, j: \tilde{T} \rightarrow \tilde{Y}, r: \tilde{W} \rightarrow \tilde{X}, \quad s: \tilde{Y} \rightarrow \tilde{X} . \\
0 \rightarrow H_{k}(\tilde{T}) \stackrel{i_{*}-j_{*}}{\longrightarrow} H_{k}(\tilde{W}) \oplus H_{k}(\tilde{Y}) \stackrel{r_{*}+s_{*}}{\longrightarrow} H_{k}\left(\tilde{X}_{M}\right) \stackrel{\Delta}{\longrightarrow} \ldots \\
\ldots H_{1}(\tilde{T}) \stackrel{i_{*}-j_{*}}{\longrightarrow} H_{1}(\tilde{W}) \oplus H_{1}(\tilde{Y}) \stackrel{r_{*}+s_{*}}{\longrightarrow} H_{1}\left(\tilde{X}_{M}\right) \rightarrow 0 .
\end{gathered}
$$

Temos as relações comutativas entre as inclusões e a ação de $t \in \Lambda$ :

$$
\begin{aligned}
& i(t x)=t(i(x)) \mathrm{e} \\
& j(t x)=t(j(x)) \quad \forall x \in \tilde{T}, \\
& r(t w)=t(r(w)) \quad \forall w \in \tilde{W}, \\
& s(t y)=t(s(y)) \quad \forall y \in \tilde{Y} .
\end{aligned}
$$

Portanto os morfismos $i_{*}, j_{*}, r_{*}$ e $s_{*}$ da sequência de Mayer-Vietoris são $\Lambda$ morfismos.

Vamos usar a notação $\frac{\Lambda}{p(t)}$ para indicar $\frac{\Lambda}{p(t) \cdot \Lambda}$.

Podemos fazer um estudo em separado no caso de superfícies de dimensão $k=2$ e de genus $g$, onde primeiramente devemos observar que:

Como $\Lambda_{n}$-módulos $\left(\Lambda_{n}=\mathbb{Z}\left[t^{n}, t^{-n}\right]\right)$ temos os isomorfismos: 


$$
\begin{gathered}
H_{0}\left(T_{0}\right) \cong \frac{\Lambda_{n}}{t^{n}-1} ; \quad H_{1}\left(T_{0}\right) \cong \frac{\left(\Lambda_{n}\right)^{2 g}}{t^{n}-1} ; \\
H_{2}\left(T_{0}\right) \cong \frac{\Lambda_{n}}{t^{n}-1} ; \quad H_{3}\left(T_{0}\right) \cong 0 .
\end{gathered}
$$

\subsection{Análise da sequência no caso $k=2$}

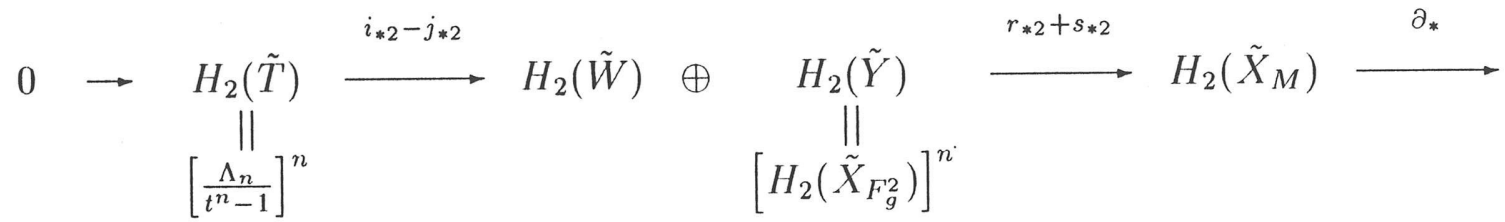

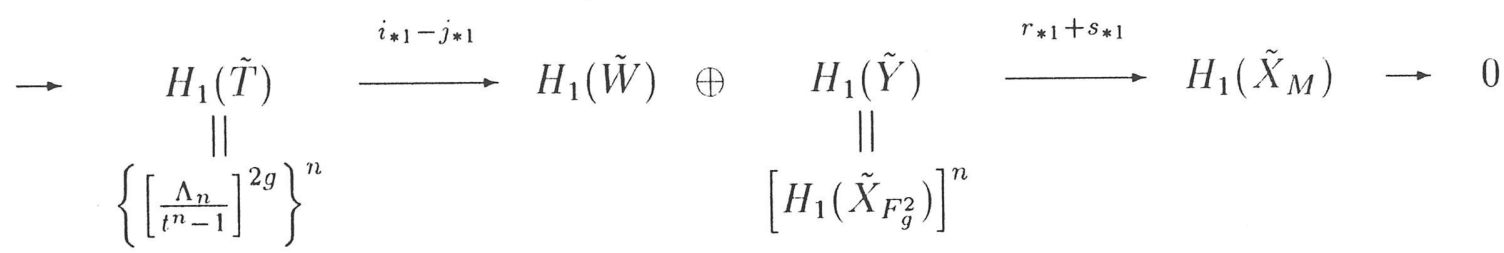

Primeiramente devemos notar que a aplicação $j_{* 2}: H_{2}(\tilde{T}) \rightarrow H_{2}(\tilde{Y})$ é um homomorfismo nulo, pois os 2-ciclos que representam o gerador de $\mathrm{H}_{2}(\tilde{T})$ quando vistos em $\tilde{Y}$ são bordo de um levantamento da variedade de Seifert de $F_{g}^{2}$.

Em segundo lugar quando é fornecido o mergulho inicial $F_{g}^{2} \subset S^{4}$ são dados indiretamente o $\Lambda$-módulo $H_{1}\left(\tilde{X}_{F_{g}^{2}}\right)$, o $\Lambda$-módulo $H_{1}(\tilde{T})$ e a aplicação induzida $j_{* 1}: H_{1}(\tilde{T}) \rightarrow H_{1}(\tilde{Y})=\left[H_{1}\left(\tilde{X}_{F_{g}^{2}}\right)\right]^{n}$.

Portanto (teóricamente) é conhecido o subgrupo $\operatorname{Ker} j_{* 1} \subset H_{1}(\tilde{T})$.

- Se $K$ er $j_{* 1} \equiv 0$ então $\left(i_{* 1}-j_{* 1}\right)$ é injetiva, implicando que $\partial_{*} \equiv 0$. E assim temos os $\Lambda$-módulos

$$
H_{1}\left(\tilde{X}_{M}\right) \cong \frac{\left[H_{1}\left(\tilde{X}_{F^{k}}\right)\right]^{n} \oplus H_{1}(\tilde{W})}{\operatorname{Im}\left(i_{* 1}-j_{* 1}\right)}
$$

$\mathrm{e}$ 


$$
H_{2}\left(\tilde{X}_{M}\right) \cong\left[H_{2}\left(\tilde{X}_{F_{g}^{2}}\right)\right]^{n} \oplus \frac{H_{2}(\tilde{W})}{H_{2}(\tilde{T})}
$$

Já o módulo $H_{1}(\tilde{W})$ pode ser calculado a partir do grupo $\pi_{1}(W)$, como é feito na próxima seção .

- Se $K$ er $j_{* 1} \neq 0$, procura-se $K$ er $i_{* 1}$ que depende da geometria da construção do satélite como veremos agora.

Usando a sequência espectral de Cartan-Leray do recobrimento $\tilde{W} \rightarrow W$, com o termo $E_{p, q}^{2}=H_{p}\left(\mathbb{Z}, H_{q}(\tilde{W})\right)$ convergindo para a homologia da base, obtemos através de cálculos semelhantes aos da demonstração do Lema 2 a sequência exata de $\Lambda$-módulos:

$$
\begin{aligned}
\ldots \rightarrow H_{i}(\tilde{W}) \stackrel{.(t-1)}{\longrightarrow} H_{i}(\tilde{W}) \stackrel{p_{*}}{\longrightarrow} H_{i}(W) \rightarrow \\
\quad \longrightarrow H_{i-1}(\tilde{W}) \stackrel{.(t-1)}{\longrightarrow} \ldots .
\end{aligned}
$$

Essa sequência finaliza com

$$
\begin{gathered}
H_{1}(\tilde{W}) \stackrel{{ }^{(t-1)}}{\longrightarrow} H_{1}(\tilde{W}) \stackrel{p_{*}}{\longrightarrow} H_{1}(W) \stackrel{\phi}{\longrightarrow} \\
\ldots \rightarrow H_{0}(\tilde{W}) \stackrel{.(t-1)}{\longrightarrow} H_{0}(\tilde{W}) \rightarrow H_{0}(W) .
\end{gathered}
$$

Temos $H_{0}(\tilde{W}) \cong \mathbb{Z}$ e portanto $.(t-1): H_{0}(\tilde{W}) \rightarrow H_{0}(\tilde{W})$ é aplicação nula.

Logo temos o isomorfismo

$$
\frac{H_{1}(W)}{p_{*}\left[H_{1}(\tilde{W})\right]} \cong \mathbb{Z}
$$

Portanto a sobrejeção abaixo que provem do homomorfismo de Hurewicz em dimensão um é um isomorfismo. 


$$
\mathbb{Z} \cong \frac{\pi_{1}(W)}{p_{*}\left[\pi_{1}(\tilde{W})\right]} \longrightarrow \frac{H_{1}(W)}{p_{*}\left[H_{1}(\tilde{W})\right]} \cong \mathbb{Z}
$$

Logo um caminho que seja representante de uma classe não nula em $H_{1}(W) / p_{*}\left[H_{1}(\tilde{W})\right]$ levanta-se em um caminho aberto em $\tilde{W}$. Pois se existisse um levantamento fechado de tal caminho esse levantamento determinaria uma classe de homologia em $H_{1}(\tilde{W})$ e portanto tal caminho estaria na imagem de $p_{*}$.

Vamos considerar a base $E=\left\{m_{h F}, a_{1}, \ldots, a_{2 g}\right\}$ para a homologia $H_{1}(W) \cong_{\Gamma_{0} \cup T_{0}} \mathbb{Z} \oplus \mathbb{Z}^{2 g}$, onde $m_{h F}$ é a classe do meridiano da vizinhança tubular $U$ e os demais são geradores da homologia $H_{1}\left(F_{g}^{2}\right)$.

Como os representantes de $a_{i}, 1 \leq i \leq 2 g$ se levantam em curvas fechadas em $\tilde{W}$ e o meridiano $m$ levanta-se aberto, o kernel da aplicação $H_{1}(W) \rightarrow H_{0}(\tilde{W}) \cong \mathbb{Z}$ é gerado pelos $2 g$ primitivos.

Logo $\operatorname{Im} p_{*} \cong \operatorname{Ker} \phi$ é isomorfo a $\mathbb{Z}^{2 g}$; mas ainda temos

$$
\operatorname{Im} p_{*} \cong \frac{H_{1}(\tilde{W})}{(t-1) H_{1}(\tilde{W})} .
$$

Então podemos fazer a afirmação de que $H_{1}(\tilde{W})$ tem pelo menos $2 g$ geradores como $\Lambda$-módulo !

Então existe possibilidade de $i_{* 1}: H_{1}(\tilde{T}) \rightarrow H_{1}(\tilde{W})$ ser injetora.

Observemos que existe a possibilidade de $i_{* 1}$ ser isomorfismo pois valem os isomorfismos:

$$
\begin{aligned}
& H_{1}(\tilde{T}) \otimes_{\Lambda} \mathbb{Z} \cong \mathbb{Z}^{2 g}, \\
& H_{1}(\tilde{W}) \otimes_{\Lambda} \mathbb{Z} \cong \mathbb{Z}^{2 g} .
\end{aligned}
$$

Seja $K=K e r\left(i_{* 1}+j_{* 1}\right)=\operatorname{Ker} i_{* 1} \cap K$ Ker $j_{* 1}$.

O submódulo $K$ tem como $\Lambda$-estrutura de módulo a induzida da inclusão $K \subset$ $H_{1}(\tilde{T})$, portanto valem os isomorfismos

$$
H_{2}\left(\tilde{X}_{M}\right) \cong_{\mathbb{Z}} K \oplus\left[H_{2}\left(\tilde{X}_{F_{g}^{2}}\right)\right]^{n} \oplus \frac{H_{2}(\tilde{W})}{H_{2}(\tilde{T})}
$$




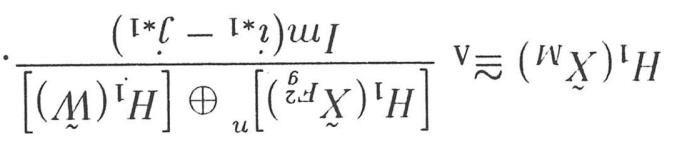




\section{Exemplos}

\section{EXEMPLO 1}

O que chamamos de toro não enodado em $S^{4}$ é exatamente o toro trivial de $\mathbb{R}^{3}$ quando visto em $S^{4}$ através das inclusões canônicas $\mathbb{R}^{3} \subset S^{3} \subset S^{4}$.

Vamos então denotar o meridiano usual desse $T^{2}$ por $a$ e denotemos por $b$ a longitude tal que existe $D^{2} \subset \mathbb{R}^{3}$ satisfazendo $D^{2} \cap T^{2}=b$. Utilizaremos também as notações $a$ e $b$ para representá-los como elementos de $\pi_{1}\left(T^{2}\right)$.

Como primeiro exemplo vamos construir uma superfície satélite $M$ do toro não enodado $T^{2} \mathrm{em} S^{4}$. A aplicação $h: T^{2} \rightarrow B_{0,2} E^{2}$ será fornecida através de

$$
\begin{gathered}
h \pi: \pi_{1} T^{2} \rightarrow \pi_{1} B_{0,2}, \\
h \pi(a)=\sigma_{1}^{3}, \quad h \pi(b)=\sigma_{1}^{3},
\end{gathered}
$$

onde estames denotando $\pi_{1} T^{2}=<a, b ; a b a^{-1} b^{-1}>$ e $\pi_{1} B_{0,2}=<\sigma_{1} ;>$.

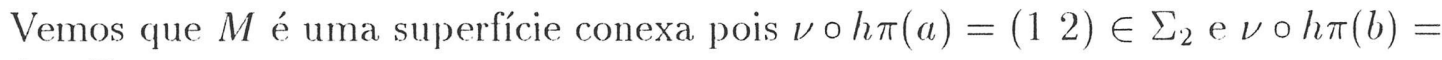
$\left(\begin{array}{ll}1 & 2\end{array}\right) \in \Sigma_{2}$.

Vamos primeiramente calcular $\pi_{1}(V-M)$ através de uma decomposição adequada de $V-M$ visto como espaço total do fibrado

$$
\xi:\left(D^{2}-2 \text { pontos }\right) \longrightarrow V-M \stackrel{p}{\longrightarrow} T^{2} .
$$

Vemos também que neste caso $M$ é recobrimento de duas folhas de $T^{2}$ e que para cada uma das curvas $a$ e $b$ de $T^{2}$ temos por restrição um braid fechado dado por $\left(\sigma_{1}\right)^{3}$ que é seu espaço total de recobrimento.

Seja $T^{2}$ o toro trivial de $\mathbb{R}^{3}$.

Considere os geradores canônicos $[a],[b]$ de $H_{1}\left(T^{2}\right)$.

Considere a longitude $b$ como sendo igual a união pelo bordo de dois espaços $J$ e $J^{\prime}$, ambos homeomorfos a um intervalo fechado, $b=J \cup_{\partial} J^{\prime}$.

Chamemos os extremos de $J$ por $j_{0}$ e $j_{1}$.

Podemos considerar sem perda de generalidade que o braid que recobre o meridiano $b$, é no trecho que recobre o espaço $J$ um braid trivial, isto é, seu "enlaçamento efetivo" está no trecho que recobre o espaço $J^{\prime}$. 
Vamos considerar como subespaço $B$ do espaço total $W=V-M$ de $\xi$ o espaço formado pelos pontos que recobrem $J \times a$.

Vamos considerar no espaço que recobre $\left\{j_{0}\right\} \times a$ um ponto $x_{0}$, no espaço que recobre $\left\{j_{1}\right\} \times a$ um ponto $x_{1}$, e uma curva $S$ unindo $x_{0}$ a $x_{1}$, inteiramnte contida em $B$, mas cuja projeção $p(S) \subset T^{2}$ seja exatamente o espaço $J$.

Vamos considerar como subespaço $A$ do espaço total $W$ de $\xi$ a união do espaço que recobre $J^{\prime} \times a$ com a curva $S$.

Temos a decomposição $W=A \cup B$.

A interseç̧ão $A \cap B$ é formada pela união dos espaços que recobrem $\left\{j_{0}\right\} \times a$ e $\left\{j_{1}\right\} \times a$ com a curva $S$.

Vamos usar o teorema de Van-Kampem para essa decomposição de $W$. Para isso fixemos um ponto base $z$ como sendo o ponto $x_{0}$ escolhido anteriormente.

Vamos calcular $\pi_{1}(B)$.

Considere uma curva fechada $L$ passando pelo ponto base $z$ de modo que $p(L)$ seja igual a curva $\left\{j_{0}\right\} \times a$.

O espaço $B$ tem o mesmo tipo de homotopia do toro sólido $L \times \stackrel{\circ}{D^{2}}$ de onde retiramos a trança fechada $\left(\sigma_{1}\right)^{3}$.

Vamos calcular $\pi_{1}(B)$ usando o método de [5] . Para isso devemos considerar curvas fechadas $x$ e $y$ com ponto base $z$ que são homotópicas aos meridianos dos dois fios da trança $\left(\sigma_{1}\right)^{3}$ que recobre $\left\{j_{0}\right\} \times a$.

Com o auxilio da figura 1 calculamos as seguintes conjugações : $L x L^{-1}=x^{-1} y^{-1} x^{-1} y x y x$ e $L y L^{-1}=x^{-1} y^{-1} x y x$ e deste modo podemos escrever:

$$
\pi_{1}(B)=<L, x, y ; \quad L x L^{-1}=x^{-1} y^{-1} x^{-1} y x y x, \quad L y L^{-1}=x^{-1} y^{-1} x y x>.
$$

Para calcular $\pi_{1}(A)$ devemos considerar uma curva fechada $K$ passando pelo ponto base $z$ de modo que a projeção $p(K) \subset T^{2}$ seja igual a curva $\left\{j_{0}\right\} \times a$.

Vamos considerar duas curvas fechadas $p$ e $q$ com ponto base $z$ que são homotópicas aos meridianos dos dois fios da trança $\left(\sigma_{1}\right)^{3}$ que recobre $\left\{j_{0}\right\} \times a$.

O subespaço $A$ tem o mesmo tipo de homotopia de um toro sólido $K \times \stackrel{0}{D^{2}}$ de onde se retirou uma trança fechada $\left(\sigma_{1}\right)^{3}$ e no qual foi colado uma alça $S$ homeomorfa 
a um intervalo fechado, através de seus extremos.

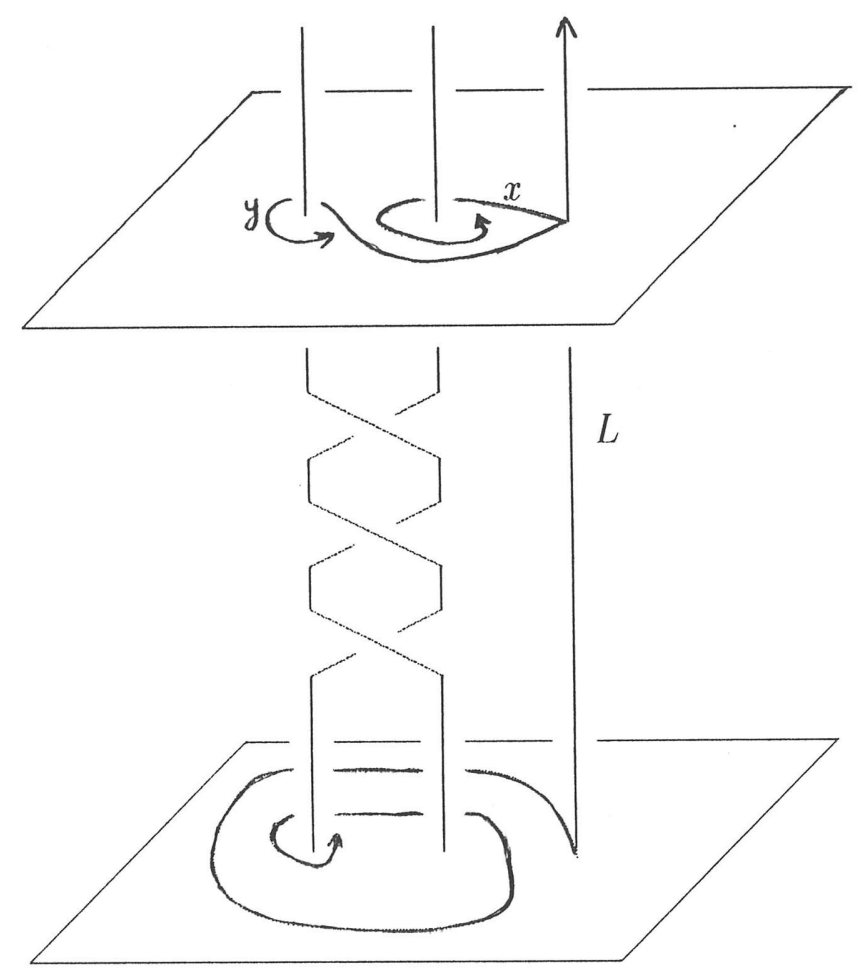

Figura 1:

Calculamos as conjugações : $K p K^{-1}=p^{-1} q^{-1} p^{-1} q p q p$, e $K q K^{-1}=p^{-1} q^{-1} p q p$.

Não podemos esquecer que $\pi_{1}(A)$ tem além de $K, p$ e $q$ um outro gerador que é livre, representado por uma curva fechada $w$ que passa pelo ponto $z$ de modo que a projeção $p(w) \subset T^{2}$ é igual ao meridiano $b$.

Assim

$$
\pi_{1}(A)=<K, p, q, w ; K p K^{-1}=p^{-1} q^{-1} p^{-1} q p q p, \quad K q K^{-1}=p^{-1} q^{-1} p q p>
$$

O subespaço $A \cap B$ tem o mesmo tipo de homotopia de dois toros sólidos 
conectados através de um segmento de reta, de onde se retiraram respectivamente duas tranças fechadas $\left(\sigma_{1}\right)^{3}$.

Vamos considerar como antes, no espaço que recobre $\left\{j_{0}\right\} \times a$ as curvas $L, x$ e $y$.

Vamos considerar no espaço que recobre $\left\{j_{1}\right\} \times a$ curvas fechadas $L^{\prime}, x^{\prime}$ e $y^{\prime}$ obtidas de modo semelhante a $L, x$ e $y$, isto é, $L^{\prime}$ é uma curva fechada que passa por $x_{1}$ e é tal que sua projeção $p\left(L^{\prime}\right) \subset T^{2}$ é igual a longitude $\left\{j_{1}\right\} \times a$ e as curvas $x^{\prime}$ e $y^{\prime}$ são curvas com ponto base $x_{1}$ que são homotópicas aos meridianos dos dois fios que compoem a trança $\left(\sigma_{1}\right)^{3}$ que está sobre $\left\{j_{1}\right\} \times a$.

Consideremos agora as curvas com ponto base $z$ :

$$
\bar{L}=S^{-1} L^{\prime} S, \quad \bar{x}=S^{-1} x^{\prime} S, \text { e } \bar{y}=S^{-1} y^{\prime} S .
$$

Agora podemos escrever a igualdade:

$$
\begin{gathered}
\pi_{1}(A \cap B)=<L, x, y, \bar{L}, \bar{x}, \bar{y} ; \quad L x L^{-1}=x^{-1} y^{-1} x^{-1} y x y x, L y L^{-1}=x^{-1} y^{-1} x y x \\
\\
\bar{L} \bar{x} \bar{L}^{-1}=\bar{x}^{-1} \bar{y}^{-1} \bar{x}^{-1} \bar{y} \bar{x} \bar{y} \bar{x}, \quad \bar{L} \bar{y}^{-1} \bar{L}^{-1}=\bar{x}^{-1} \bar{y}^{-1} \bar{x} \bar{y} \bar{x}>.
\end{gathered}
$$

O homomorfismo induzido pela inclusão $i_{1}: \pi_{1}(A \cap B) \rightarrow \pi_{1}(B)$ é dado por $i_{1}(L)=L, i_{1}(x)=x, i_{1}(y)=y, i_{1}(\bar{L})=L, i_{1}(\bar{x})=x, i_{1}(\bar{y})=y$.

O homornorfismo induzido pela inclusão $i_{2}: \pi_{1}(A \cap B) \rightarrow \pi_{1}(A)$ śatisfaz $i_{2}(L)=$ $K, i_{2}(x)=p, i_{2}(y)=q, i_{2}(\bar{L})=w K w^{-1}$.

Devemos observar que devido a posição do ponto base $z$ valem $i_{2}(\bar{x})=w p^{-1} q^{-1} p^{-1} q p q p w^{-1}$ e $i_{2}(\bar{y})=w p^{-1} q^{-1} p q p w^{-1}$.

Então aplicando o teorema de Van-Kampen obtemos:

$$
\begin{aligned}
& \pi_{1}(A \cap B) \stackrel{\longrightarrow}{i_{1}(B)} \pi_{1}(A) \longrightarrow \pi_{1}(A \cup B)=\pi_{1}(V-M) \\
& \pi_{1}(V-M)=<L, x, y, K, p, q, w ; L x L^{-1}=x^{-1} y^{-1} x^{-1} y x y x, \quad L y L^{-1}=x^{-1} y^{-1} x y x, \\
& K p K^{-1}=p^{-1} q^{-1} p^{-1} q p q, K q K^{-1}=p^{-1} q^{-1} p q p, L=K, x=p, y=q, L=w K w^{-1},
\end{aligned}
$$




$$
\begin{aligned}
& x=w p^{-1} q^{-1} p^{-1} q p q p w^{-1} \\
& y=w p^{-1} q^{-1} p q p w^{-1}>
\end{aligned}
$$

Portanto temos:

$$
\begin{gathered}
\pi_{1}(V-M)=<L, x, y, w ; \quad L x L^{-1}=x^{-1} y^{-1} x^{-1} y x y x, \quad L y L^{-1}=x^{-1} y^{-1} x y x \\
L=w L w^{-1}, \quad w^{-1} x w=x^{-1} y^{-1} x^{-1} y x y x, \quad w^{-1} y w=x^{-1} y^{-1} x y x>
\end{gathered}
$$

Aplicando o teorema de Van-Kampen novamente:

$$
\begin{aligned}
& \pi_{1}(\partial V) \underset{j_{1}}{j_{1}} \pi_{1}(\bar{V}-M) \\
& \pi_{1}\left(S^{4}-V\right)=<m_{F} ;>\text { pois o toro é o trivial. } \\
& \pi_{1}(\partial V)=<a, b, o ; \quad a b=b a, \quad a o=o a, \quad b o=o b>. \\
& j_{2}(a)=j_{2}(b)=0, j_{2}(o)=m_{F}, j_{1}(a)=L, j_{1}(b)=w, j_{1}(o)=x y .
\end{aligned}
$$

Portanto temos:

$$
\begin{gathered}
\pi_{1}\left(S^{4}-M\right)=<L, x, y, w, m_{F} ; \quad L x L^{-1}=x^{-1} y^{-1} x^{-1} y x y x, \quad L y L^{-1}=x^{-1} y^{-1} x y x \\
L=w L w^{-1}, \quad w^{-1} x w=x^{-1} y^{-1} x^{-1} y x y x, \quad w^{-1} y w=x^{-1} y^{-1} x y x \\
L=0, w=0, m_{F}=x y>= \\
=<x, y ; x^{-1} y^{-1} x^{-1} y x y>\cong \pi_{1}\left(S^{3}-\text { trefoil }\right) . \\
\pi_{1}\left(S^{4}-M\right) \cong \pi_{1}\left(S^{3} \text { - trefoil }\right),
\end{gathered}
$$

donde deduzimos que 


$$
H_{1}\left(\tilde{X}_{M}\right) \cong \frac{\Lambda}{<1-t+t^{2}>}
$$

$H_{1}\left(\tilde{X}_{M}\right)$ é livre de pseudonulo e portanto $e^{2}\left(H_{1}\left(\tilde{X}_{M}\right)\right)=0$ que faz cindir a sequência exata do item 4 do Teorema 1.

Após alguns cálculos como os efetuados no próximo exemplo iremos obter:

$$
H_{2}\left(\tilde{X}_{M}\right) \cong \Lambda^{2} \oplus \frac{\Lambda}{<1-t+t^{2}>}
$$

\section{EXEMPLO 2}

Considere novamente o toro $T^{2}$ não enodado em $S^{4}$ e façamos outra construção do satélite $M$ como abaixo.

Tome por exemplo a aplicação $h \pi: \pi_{1}\left(T^{2}\right) \rightarrow \pi_{i}\left(B_{0, n}\right)$ de modo que $h \pi(a)=$ $h \pi(b)=W$, onde $W$ é uma palavra nos geradores do contradomínio, de modo que $\nu \circ h \pi(a)$ seja igual a algum conjugado de $(12 \ldots n) \in \Sigma_{n}$ (notação de 5.6).

Usando a mesma decomposição de $V-M$ em subespaços $A$ e $B$ como já foi feito, e a utilização do ponto base $z$ na mesma posição anterior teremos através de cálculos semelhantes os resultados:

$$
\begin{gathered}
\pi_{1}(B)=<L, x_{1}, \ldots, x_{n} ; L x_{i} L^{-1}=A_{i} x_{W(i)} A_{i}^{-1}, \quad 1 \leq i \leq n>, \\
\pi_{1}(A)=<K, p_{1}, \ldots, p_{n}, w ; K p_{j} K^{-1}=A_{j} p_{W(j)} A_{j}^{-1}, \quad 1 \leq j \leq n>
\end{gathered}
$$

e

$$
\begin{gathered}
\pi_{1}(A \cap B)=<L, x_{1}, \ldots, x_{n}, \bar{L}, \bar{x}_{1}, \ldots, \bar{x}_{n} ; L x_{i} L^{-1}=A_{i} x_{W(i)} A_{i}^{-1} \\
\bar{L}_{i} \bar{x}_{i}^{-1}=\bar{A}_{i} \bar{x}_{W(i)} \bar{A}_{i}^{-1}, 1 \leq i \leq n>
\end{gathered}
$$

Aqui estamos usando a notação $A_{i}$ para indicar uma palavra nas letras $x_{j}, \quad 1 \leq j \leq n$, determinada pela trança $W, \bar{A}_{i}$ para indicar palavra semelhante nas letras $\bar{x}_{j}$, e a notação $W(i)$ para indicar a posição final do $i$-ésimo fio da trança $W$. 
O homomorfismo induzido pela inclusão $i_{1}: \pi_{1}(A \cap B) \rightarrow \pi_{1}(B)$ é dado por:

$$
i_{1}(L)=L, i_{1}\left(x_{i}\right)=x_{i}, i_{1}(\bar{L})=L, i_{1}\left(\bar{x}_{i}\right)=x_{i}, \quad 1 \leq i \leq n .
$$

O homomorfismo induzido pela inclusão $i_{2}: \pi_{1}(A \cap B) \rightarrow \pi_{1}(A)$ satisfaz:

$$
i_{2}(L)=K, i_{2}\left(x_{i}\right)=p_{i}, \quad 1 \leq i \leq n .
$$

E devido a posição do ponto base $z$ valem as inclusões:

$$
i_{2}(\bar{L})=w K w^{-1}, \quad i_{2}\left(\bar{x}_{i}\right)=w A_{i} p_{W(i)} A_{i}^{-1} w^{-1}, 1 \leq i \leq n .
$$

Então aplicando o teorema de Van-Kampen obtemos:

$$
\begin{gathered}
\pi_{1}(V-M)=<L, x_{i}, w: L x_{i} L^{-1}=A_{i} x_{W(i)} A_{i}^{-1}, L=w L w^{-1}, \\
w^{-1} x_{i} w=A_{i} x_{W(i)} A_{i}^{-1}, \quad 1 \leq i \leq n>.
\end{gathered}
$$

Como anteriormente

$$
\pi_{1}\left(S^{4}-V\right)=<m_{F} ;>
$$

pois o toro é não enodado e

$$
\pi_{1}(\partial \bar{V})=\langle a, b, o ; a b=b a, a o=o a, b o=o b>.
$$

A caracterização do morfismo $j_{1}: \pi_{1}(\partial \bar{V}) \rightarrow \pi_{1}(\bar{V}-M)$ é dada por:

$$
j_{1}(a)=L, \quad j_{1}(b)=w, \quad j_{1}(o)=x_{1} x_{2} \ldots x_{n-1} x_{n} .
$$

A caracterização do morfismo $j_{2}: \pi_{1}(\partial \bar{V}) \rightarrow \pi_{1}\left(S^{4}-V\right)$ é dada por:

$$
j_{2}(a)=j_{2}(b)=0, \quad j_{2}(o)=m_{F} .
$$

Aplicando o teorema de Van-Kampen obtemos: 


$$
\pi_{1}\left(S^{4}-M\right)=<x_{i}: \quad x_{i}=A_{i} x_{W(i)} A_{i}^{-1}, \quad 1 \leq i \leq n>
$$

Chamemos o nó proveniente da trança fechada $W$ por $K(W)$ e denotemos seu polinômio de Alexander na variável $t$ por $p(t)$.

Segundo Birman [5] $\pi_{1}\left(S^{4}-M\right)$ tem a mesma apresentação de $\pi_{1}\left(S^{3}-K(W)\right)$.

Portanto temos o módulo de Alexander livre de pseudonulo

$$
H_{1}\left(\tilde{X}_{M}\right) \cong \frac{\Lambda}{<p(t)>}
$$

Usando a Proposição 1.18 de [26] conseguimos deduzir que

$$
e^{2}\left(H_{1}\left(\tilde{X}_{M}\right)\right)=0
$$

Usando o isomorfismo do Lema 7 também obtemos $e^{2}\left[H_{1}\left(\tilde{X}_{M}, \partial \tilde{X}_{M}\right)\right]=0$.

Portanto pelo Teorema 1 temos:

$$
0 \rightarrow e^{1}\left[H_{1}\left(\tilde{X}_{M}\right)\right] \rightarrow \overline{H_{2}\left(\tilde{X}_{M}, \partial \tilde{X}_{M}\right)} \rightarrow H_{2}\left(\tilde{X}_{M}\right)^{*} \rightarrow 0 .
$$

Logo

$$
\overline{H_{2}\left(\tilde{X}_{M}, \partial \tilde{X}_{M}\right)} \cong e^{1}\left[H_{1}\left(\tilde{X}_{M}\right)\right] \oplus \Lambda^{2}
$$

Tomamos a resolução

$$
\Lambda \stackrel{p(t)}{\longrightarrow} \Lambda \longrightarrow H_{1}\left(\tilde{X}_{M}\right) \longrightarrow 0 .
$$

Usando o funtor Hom $(-, \Lambda)$ temos

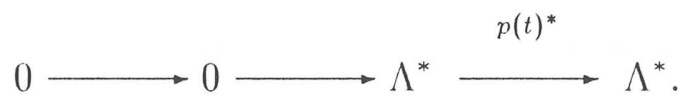


E portanto

$$
e^{1}\left[H_{1}\left(\tilde{X}_{M}\right)\right] \cong \frac{\Lambda}{p(t)} \cong e^{1}\left[H_{1}\left(\tilde{X}_{M}, \partial \tilde{X}_{M}\right)\right]
$$

Logo

$$
\overline{H_{2}\left(\tilde{X}_{M}, \partial \tilde{X}_{M}\right)} \cong \frac{\Lambda}{p(t)} \oplus \dot{\Lambda}^{2}
$$

e assim

$$
H_{2}\left(\tilde{X}_{M}, \partial \tilde{X}_{M}\right)^{*} \cong \Lambda^{2} \text {. }
$$

Usando a outra sequência do Teorema 1 temos:

$$
0 \rightarrow \frac{\Lambda}{p(t)} \rightarrow \overline{H_{2}\left(\tilde{X}_{M}\right)} \rightarrow \Lambda^{2} \rightarrow 0
$$

Logo

$$
H_{2}\left(\tilde{X}_{M}\right) \cong \Lambda^{2} \oplus \frac{\Lambda}{<p(t)\rangle}
$$

\section{EXEMPLO 3}

Se nas mesmas condições anteriores construirmos um outro satélite através de:

$$
h \pi: \pi_{1}\left(T^{2}\right) \rightarrow \pi_{1}\left(B_{0, n}\right), \quad h \pi(a)=W, \quad h \pi(b)=i d
$$

$\operatorname{com} \nu \circ h \pi(b)=(12 \ldots n) \in \Sigma_{n}$.

Se proseguirmos fazendo a mesma divisão do espaço $V-M$ e utilizando o Teorema de Van-Kampen, obteremos

$$
H_{1}\left(\tilde{X}_{M}\right) \cong \frac{\Lambda}{<p(t)>}, \text { e } H_{2}\left(\tilde{X}_{M}\right) \cong \Lambda^{2} \oplus \frac{\Lambda}{<p(t)>}
$$

Pois teremos os grupos:

$$
\begin{gathered}
\pi_{1}(B)=<L, x_{i} ; L x_{i}=x_{i} L, 1 \leq i \leq n>, \\
\pi_{1}(A)=<K, p_{i}, w ; K p_{i} K^{-1}=A_{i} p_{W(i)} A_{i}^{-1}, \quad 1 \leq i \leq n>,
\end{gathered}
$$




$$
\begin{gathered}
\pi_{1}(A \cap B)=<L, x_{i}, \bar{L}, \bar{x}_{i} ; L x_{i} L^{-1}=A_{i} x_{W(i)} A_{i}^{-1} \\
\bar{L}_{\bar{x}_{i}} \bar{L}_{i}=\bar{A}_{i} \bar{x}_{W(i)} \bar{A}_{i}^{-1}, \quad 1 \leq i \leq n>
\end{gathered}
$$

Teremos os homomorfismos induzidos pelas inclusões:

$$
i_{1}(L)=L, i_{1}\left(x_{i}\right)=x_{i}, i_{1}(\bar{L})=L, i_{1}\left(\bar{x}_{i}\right)=x_{i}
$$

e

$$
i_{2}(L)=K, i_{2}\left(x_{i}\right)=p_{i}, i_{2}(\bar{L})=w K w^{-1}, i_{2}\left(\bar{x}_{i}\right)=w p_{i} w^{-1} .
$$

Aplicando o teorema de Van-Kampen teremos:

$\pi_{1}(V-M)=<L, x_{i}, w ; L x_{i} L^{-1}=A_{i} x_{W(i)} A_{i}^{-1}, L x_{i}=x_{i} L, L=w L w^{-1}, x_{i}=w x_{i} w^{-1}>$.

E finalmente podemos aplicar o teorema de Van-Kampen com os grupos:

$$
\pi_{1}\left(S^{4}-V\right)=<m_{F} ; \quad>, \quad \pi_{1}(\partial \bar{V})=<a, b, o ; a b=b a, a o=o a, b o=o b>,
$$

com homomorfismos induzidos pelas inclusões dados por:

$$
j_{2}(a)=j_{2}(b)=0, \quad j_{2}(o)=m_{F}, \quad j_{1}(a)=L, \quad j_{1}(b)=w, \quad j_{1}(o)=x_{1} \ldots x_{n} .
$$

Calculamos:

$$
\pi_{1}\left(S^{4}-M\right)=<x_{i} ; x_{i}=A_{i} x_{W(i)} A_{i}^{-1}, \quad 1 \leq i \leq n>
$$

que é a apresentação do complementar em $S^{3}$ do nó $K(W)$. 


\section{O satélite $T^{2}$-torus-nó}

Os exemplos apresentados na seção anterior podem ser comparados com um tipo de mergulho do toro $T^{2}$ na esfera $S^{4}$ chamado por Iwase [14] de $T^{2}$-nó.

Definição 6 Seja $M^{n}$ uma variedade n-dimensional e $N^{n-2}$ uma variedade (n-2)dimensional. Uma subvariedade $K$ em $M$ é dita $N^{n-2}$-nó em $M$ se $K$ é difeomorfa a $N^{n-2}$. Sejam $K$ e $K^{\prime}$ dois $N^{n-2}$-nós em $M$. K e $K^{\prime}$ são ditos equivalentes se existir um difeomorfismo $h:(M, K) \rightarrow\left(M, K^{\prime}\right)$.

Os mergulhos nos quais estamos interessados são os $T^{2}$-nós em $S^{4}$.

Lembremos que um $T^{1}$-nó $K$ em $S^{3}$ é dito ser um "torus-nó" se $K$ está essencialmente mergulhado em $\partial N(U)$, onde $U$ é um $T^{1}$-nó não enodado em $S^{3}$ e $N(U)$ denota uma vizinhança tubular do mesmo.

Definição 7 Seja $M$ uma variedade 3-dimensional e $F$ uma superfície compacta, conexa, propriamente mergulhada em $M$. Dizemos que $F$ é incompressivel em $M$ se nenhuma das seguintes condições é satisfeita:

A) F é uma D-esfera que borda uma 3-célula em $M$;

B) $F$ é uma 2-célula e: Ou $F \subset \partial M$ ou existe uma 3-célula $X \subset M$ com $\partial X \subset(F \cup \partial M)$;

C) Existe uma 2-célula $D \subset M$ com $D \cap F=\partial D$ e com $\partial D$ não contrátil em $F$.

Definição 8 Diremos que um $T^{2}$-nó $K \mathrm{em} S^{4}$ é um "torus-nó" se $K$ está incompressivelmente mergulhado em $\partial N(U)$, onde $U$ é um $T^{2}$-nó não enodado.

Lema 29 Sejam $K$ e $K^{\prime}$ dois $T^{2}$ mergulhados incompressiveis em $T^{3}\left(T^{3}=S^{1} \times\right.$ $\left.S^{1} \times S^{1}\right)$ tal que $[K]=\left[K^{\prime}\right]$ em $H_{2}\left(T^{3}\right)$. Então existe uma isotopia ambiente que leva $K \mathrm{em} K^{\prime}$.

Dem. Este é o Lema 2.5 de Iwase [14].

Motesinos em [25] define um espaço chamado "twin" do seguinte modo.

Considere $S^{4}$ como a compactificação $S^{4}=\mathbb{R}^{4} \cup\{\infty\}$, com a orientação canônica de $\mathbb{R}^{4}$. 
Em $S^{4}=\mathbb{R}^{4} \cup\{\infty\}$ tome o conjunto

$$
E^{4}=\left\{\left(x_{1}, x_{2}, x_{3}, x_{4}\right) \in \mathbb{R}^{4} \mid x_{1}^{2}+x_{2}^{2} \leq 1 \text { ou } x_{3}^{2}+x_{4}^{2} \leq 1\right\} \cup V(\infty),
$$

com orientação induzida de $S^{4}$.

Estamos denotando uma vizinhança de $\{\infty\}$ por $V(\infty)$.

Então $E^{4}$ é uma vizinhança regular da união dos dois seguintes planos coordenados

$$
R=\left(\mathbb{R}^{2} \times(0,0)\right) \cup\{\infty\} \text { e } S=\left((0,0) \times \mathbb{R}^{2}\right) \cup\{\infty\},
$$

considerados com orientações induzidas das coordenadas de $\mathbb{R}^{4}$. $R$ e $S$ se interceptam transversalmente com sinal positivo em $\{(0,0,0,0)\}$ e com sinal negativo em $\{\infty\}$.

Montesinos chama de "twin" a tripla $\left(E^{4}, R, S\right)$.

O complemento $S^{4}-i n t E^{4}$ é uma vizinhança regular do toro

$$
T^{2}=\left\{\left(x_{1}, x_{2}, x_{3}, x_{4}\right) \in \mathbb{R}^{4} \mid x_{1}^{2}+x_{2}^{2}=2=x_{3}^{2}+x_{4}^{2}\right\} .
$$

Identificando $S^{1}$ com o quociente $\mathbb{R} / \mathbb{Z}$ e denotando por $T^{3}$ o toro tridimensional $S^{1} \times S^{1} \times S^{1}$, vemos que $\partial E^{4}$ é homeomorfo a $T^{3}$.

Um meridiano de $R$ é uma curva simples orientada de $T^{3}$ tal que é bordo de um disco orientado propriamente mergulhado em $E^{4}$ que intercepta $R$ com sinal positivo transversalmente em um único ponto. Do mesmo modo definimos um meridiano de $S$. Vamos chamá-los respectivamente de $r$ e $s$.

Seja $U$ um $T^{2}$-nó não enodado em $S^{4}$, por exemplo

$$
U=\left\{\left(x_{1}, x_{2}, x_{3}, x_{4}\right) \in \mathbb{R}^{4} \mid x_{1}^{2}+x_{2}^{2}=2=x_{3}^{2}+x_{4}^{2}\right\} .
$$

Então segundo Montesinos $\overline{S^{4}-N(U)}$ é um "twin". Vamos denotar o twin pelo símbolo $T w$. Considere como antes as esferas $R$ e $S$. Elas são geradoras de $H_{2}(T w)$, veja Lema 1.

Considere os meridianos $r$ e $s$ definidos anteriormente. Suas classes de homologia em $H_{1}(\partial(T w))$ são bem definidas. Tome um círculo $l$ em $\partial(T w)$ tal que $\{[l],[r],[s]\}$ é uma base orientada para $H_{1}(\partial(T w))$. Segundo Iwase [14] duas escolhas de "l" são relacionadas por um difeomorfismo que deixa fixo os meridianos $r$ e $s$. 
Considere a variedade $D^{2} \times T^{2} \cong D^{2} \times S^{1} \times S^{1}$. Denotemos por $\bar{l}, \bar{r}$ e $\bar{s}$ respectivamente os círculos $\partial D^{2} \times\{*\} \times\{*\},\{*\} \times S^{1} \times\{*\}$ e $\{*\} \times\{*\} \times S^{1}$ em $\partial\left(D^{2} \times T^{2}\right)$.

Observemos que $S^{4}=T w \cup_{f}\left(D^{2} \times T^{2}\right)$ onde $f: \partial\left(D^{2} \times T^{2}\right) \rightarrow$ é um difeo tal que $f_{*}[\bar{l}]=[l], f_{*}[\bar{r}]=[r]$ e $f_{*}[\bar{s}]=[s]$.

Observemos que $\left(D^{2} \times T^{2}\right) \cap T w=\partial T w$.

Vamos assumir que $K$ é um $T^{2}$-torus-nó contido em $\partial(T w)$.

Vamos denotar $K$ por $K\left(p, q, q^{\prime}\right)$ se $[K]=p \cdot[(r \times s)]+q \cdot[(s \times l)]+q^{\prime} \cdot[(l \times r)]$ em $H_{2}(\partial(T w))$, onde $p, q, q^{\prime} \in \mathbb{Z}$.

Note que pelo lema anterior $\left(p, q, q^{\prime}\right)$ determina o tipo do nó $K \subset \partial(T w)$.

Analisando os possíveis difeomorfismos $h: \partial(T w) \rightarrow \partial(T w)$ que se estendem a difeomorfismos $\bar{h}: S^{4} \rightarrow S^{4}$, Iwase [14] consegue mostrar:

Lema 30 Qualquer $T^{2}$-torus-nó é equivalente a um e apenas um dos seguintes:

A) $K(p, q, 0), 1<p<q, \operatorname{mdc}(p, q)=1$;

B) $K(p, q, q), 1<p<q, \operatorname{mdc}(p, q)=1$;

C) $T^{2}$-nó não enodado.

Dem. Este é o Lema 2.9 de Iwase [14].

Vamos descrever esses $T^{2}$-torus-nós $K\left(p, q, q^{\prime}\right)$ de outra maneira.

Pense $S^{4}$ como $\partial\left(D^{2} \times D^{3}\right)=S^{1} \times D^{3} \cup D^{2} \times S^{2}$.

Considere o nó trivial orientado $k_{0}$, imagem de um megulho $g: S^{1} \rightarrow$ int $D^{3}$, onde $D^{3}$ é o disco do primeiro fator da união $S^{1} \times D^{3} \cup D^{2} \times S^{2}=S^{4}$.

Claramente vemos que $\exists D_{0}^{2} \subset D^{3}$ tal que $\partial D_{0}^{2}=k_{0}$.

Para nossos cálculos o representante da classe de mergulhos triviais de $T^{2} \mathrm{em} \mathrm{S}^{4}$ que vamos fixar é

$$
U=S^{1} \times k_{0} \subset S^{1} \times D^{3} \subset S^{1} \times D^{3} \cup_{\partial} D^{2} \times S^{2}=S^{4} .
$$

Claramente vemos que $S^{1} \times D_{0}^{2}$ satisfaz $\partial\left(S^{1} \times D_{0}^{2}\right)=U$. 
Fixemos $r$ como sendo $r=k_{0}$, tomado com a mesma orientação de $k_{0}$.

Tomemos $s$ como sendo a $S^{1}$ orientada do cartesiano $S^{1} \times k_{0}$. E finalmente para completar a base $\{[l],[r],[s]\}$ de $H_{1}\left(\partial\left[S^{4}-\operatorname{int} N(U)\right]\right)$ fixemos como $l$ o meridiano em $D^{3}$ do nó trivial $k_{0}$.

Considere um $T^{1}$-torus-nó $k$ do tipo $(p, q)$, denotado $k(p, q)$ no toro $l \times r \subset D^{3}$, vamos fixar como representante desta classe o nó orientado $\alpha$ que recobre $l$ um número de vezes igual a $-q$ e que recobre $r$ um número de vezes igual a $p$. Ou seja $\alpha$ é um nó do tipo $(-q, p)$, já que as classes de equivalência $k(p, q)$ e $k(-q, p)$ são iguais.

Chamamos de "spun-torus" de $k(p, q)$ ao $T^{2}$-nó dado pelo cartesiano $S^{1} \times k(p, q)$ contido no fator $S^{1} \times D^{3}$ da decomposição acima de $S^{4}$. Denotemos o spun-torus de $k(p, q)$ por $S(k(p, q))$.

Consideremos para nossos cálculos o representante $s \times \alpha$ da classe $S(k(p, q))$ e fixemos a classe de orientação $[\alpha \times s] \in \mathrm{H}_{2}(S(k(p, q)))$.

Seja $\tau$ o automorfismo de $S^{1} \times S^{2}$ definido por $\tau(\theta, x)=(\theta, \rho(\theta) x)$, onde $\rho(\theta)$ é a rotação de $S^{2}$ de ângulo $\theta$ ao redor de seus polos.

Essa aplicação $\tau$ se estende naturalmente para um automorfismo $\bar{\tau}: S^{1} \times D^{3} \rightarrow S^{1} \times D^{3}$.

Chamamos de "n-twist spun torus" de $k(p, q)$ a imagem de $S^{1} \times k(p, q) \subset S^{1} \times D^{3}$ quando considerado em

$$
S^{4}=S^{1} \times D^{3} \cup_{\tau^{n}} D^{2} \times S^{2} .
$$

Denotemos o 1-twist spun torus de $k(p, q)$ por $\tilde{S}(k(p, q))$.

Consideremos para nossos cálculos o representante $s \times \alpha$ da classe $\tilde{S}(k(p, q))$ e fixemos a classe de orientação $[\alpha \times s] \in H_{2}(\tilde{S}(k(p, q)))$.

Vamos verificar a seguinte afirmação feita por Iwase:

Afirmação : $K(p, q, 0)=S(k(p, q))$ e $K(p, q, q)=\tilde{S}(k(p, q))$.

Vamos considerar pontos arbitrários $l_{0} \in l, r_{0} \in r$ e $s_{0} \in s$.

Vamos também considerar as seguintes projeções :

$$
\begin{aligned}
& \pi_{12}: \partial(T w) \longrightarrow S^{1} \times S^{1} \times\{*\}, \\
& \pi_{13}: \partial(T w) \longrightarrow S^{1} \times\{*\} \times S^{1},
\end{aligned}
$$


$\pi_{23}: \partial(T w) \longrightarrow\{*\} \times S^{1} \times S^{1}$.

Devemos observar como são as três seguintes intersecções :

- $\left(l \times r \times\left\{s_{0}\right\}\right) \cap S(k(p, q))$.

Esta intersecção consiste de um nó $\alpha(=k(-q, p))$ no toro $l \times r$.

- $\left(l \times\left\{r_{0}\right\} \times s\right) \cap S(k(p, q))$.

Esta intersecção consiste de uma trança trivial com $p$ fios ao longo de $s$.

- $\left(\left\{l_{0}\right\} \times r \times s\right) \cap S(k(p, q))$.

Esta intersecção consiste de uma trança trivial de $q$ fios ao longo de $s$.

Então vemos que a imagem do spun torus pela projeção no segundo e terceiro fator satisfaz

$$
\left[\pi_{23}(S(k(p, q)))\right]=p \cdot[r \times s] \in H_{2}(\partial(T w)) .
$$

Vemos também que a imagem do spun torus pela projeção no primeiro e terceiro fator satiafaz

$$
\left[\pi_{13}(S(k(p, q)))\right]=q \cdot[s \times l] \in H_{2}(\partial(T w)) .
$$

Vemos finalmente que a imagem do spun torus pela projeção no primeiro e segundo fator satisfaz

$$
\left[\pi_{12}(S(k(p, q)))\right]=0 .[l \times r] \in H_{2}(\partial(T w)) .
$$

E portanto

$$
S(k(p, q))=K(p, q, 0) .
$$

Devemos novamente observar como são as três seguintes intersecções :

- $\left(l \times r \times\left\{s_{0}\right\}\right) \cap \tilde{S}(k(p, q))$.

Como no caso anterior, essa intersecção consiste de um nó $k(-q, p)$ no toro $l \times r$. 
- $\left(l \times\left\{r_{0}\right\} \times s\right) \cap \tilde{S}(k(-q, p))$.

Esta intersecção consiste de uma trança de $p$ fios ao longo de $s$, mas que não é mais uma trança trivial como no caso anterior. Devemos perceber que a medida que percorremos a curva orientada $s$ (que é $S^{1}$ !) o ponto $r_{0}$, (utilizando um abuso de linguagem), "também percorre" toda a curva $r$ uma vez. Logo o braid não é o trivial mas sim é um nó $k(p, q)$ no toro $s \times l$.

- $\left(\left\{l_{0}\right\} \times r \times s\right) \cap \tilde{S}(k(p, q))$.

Esta intersecção consiste de uma trança de $q$ fios ao longo de $s$, mas com rotação de $2 \pi$ na direção de $r$.

Então vemos que :

$$
\begin{aligned}
& {\left[\pi_{23}(\tilde{S}(k(p, q)))\right]=p \cdot[r \times s] \in H_{2}(\partial(T w)) .} \\
& {\left[\pi_{13}(\tilde{S}(k(p, q)))\right]=q \cdot[s \times l] \in H_{2}(\partial(T w)) .} \\
& {\left[\pi_{12}(\tilde{S}(k(p, q)))\right]=q \cdot[l \times r] \in H_{2}(\partial(T w)) .}
\end{aligned}
$$

E portanto

$$
\tilde{S}^{\prime}(k(p, q))=K(p, q, q) .
$$

O lema anterior pode ser reescrito na seguinte forma.

Lema 31 Todo $T^{2}$-torus-nó é equivalente a um e apenas um dos seguintes:

A) $S(k(p, q)), 1<p<q, \operatorname{mdc}(p, q)=1$,

B) $\tilde{S}(k(p, q)), 1<p<q, \operatorname{mdc}(p, q)=1$,

C) $T^{2}$-nó não enodado.

Analisando as interseç̧ões escritas anteriormente vemos que:

1. $S(k(p, q))$ é o satélite do toro não enodado $U$ obtido a partir da aplicação $h: U \rightarrow B_{0, p}$, associada a $h \pi: \pi_{1}(U) \rightarrow \pi_{1} B_{0, p}$, definida por $h \pi(r)=W_{p, q}$ e $h \pi(s)=i d$, onde $W_{p, q}$ é uma palavra nos geradores de $\pi_{1} B_{0, p}$ que designa o braid cujo fecho é o nó $k(p, q)$ ao longo de $r$. 
2. $\tilde{S}(k(p, q))$ é o satélite do toro não enodado $U$ obtido a partir da aplicação $h: U \rightarrow B_{0, p}$, associada a $h \pi: \pi_{1}(U) \rightarrow \pi_{1} B_{0, p}$, definida por $h \pi(r)=W_{p, q}$, e $h \pi(s)=W_{p, q}$.

Concluimos portanto que os $T^{2}$-torus-nós definidos por Iwase [14] são casos particulares de satélites de superfícies, onde a superfície inicial é o toro não enodado $U$ e o satélite está inteiramente contido no bordo da vizinhança tubular $N(U)$.

Então fica interessante destacar o seguinte fato:

Lema 32 Os satélites $S(k(p, q))$ e $\tilde{S}(k(p, q))$ têm os mesmos módulos de Alexander.

Dem. Reproduzindo o processo de cálculo utilizado na seção 8 obteremos para ambos os satélites acima os seguintes módulos:

$$
H_{1}(\tilde{X}) \cong \frac{\Lambda}{<\Delta(t)>}, \text { e } H_{2}(\tilde{X}) \cong \Lambda^{2} \oplus \frac{\Lambda}{<\Delta(t)>}
$$

Aqui estamos denotando o polinômio de Alexander do nó toral do tipo $(p, q)$ por

$$
\Delta(t)=\frac{(1-t)\left(1-t^{p q}\right)}{\left(1-t^{p}\right)\left(1-t^{q}\right)}
$$

Segundo Iwase [14] temos dois fatos a observar:

A) Os exteriores de $S(k(p, q))$ e de $\tilde{S}(k(p, q))$ têm o mesmo tipo de homotopia.

B) Os satélites $S(k(p, q))$ e $\tilde{S}^{\prime}(k(p, q))$ não são equivalentes pois seus exteriores não são difeomorfos. 


\section{Construção Iterada}

O processo de construção de satélites aqui apresentado pode ser repetido a partir de uma variedade satélite já obtida. Este processo é semelhante a obtenção de nós (clássicos) iterados no toro.

Como exemplo final vamos fazer uma construção em dimensão dois, utilizando os resultados e notações das seções anteriores.

Considere como antes o nó trivial

$$
k_{0} \subset D^{3} \subset S^{1} \times D^{3} \cup D^{2} \times S^{2}=S^{4}
$$

Chamamos a atenção ao fato de que em todas as etapas, as trivializações das vizinhanças tubulares dos nós utilizados para a construção iterada deverão ser as distinguidas.

No bordo de uma vizinhança tubular $V_{1} \cong T_{0} k_{0} \times D^{2}$, tomemos o nó $k(p, q)$, onde $T_{0}$ é a trivialização distinguida do nó $k_{0}$. Este nó esta contido no toro $k_{0} \times S^{1} \subset D^{3}$. Denotemos $k(p, q)$ por $k_{1}$.

Como anteriormente $S(k(p, q))=S\left(k_{1}\right)$ é obtido através de $h: T^{2} \rightarrow B_{0, p}$ associada a:

$$
\begin{gathered}
h \pi: \pi_{1}\left(T^{2}\right) \longrightarrow \pi_{1}\left(B_{0, p}\right), \\
h \pi\left(\left[k_{0}\right]\right)=W_{p, q}, \quad h \pi\left(\left[S^{1}\right]\right)=i d,
\end{gathered}
$$

onde $T^{2}$ é o toro trivial $S^{1} \times k_{0} \subset S^{4}$ e $W_{p, q}$ é uma palavra nos geradores de $\pi_{1}\left(B_{0, p}\right)$ que designa o braid fechado $k_{p, q}$.

No bordo de uma vizinhança tubular $V_{2} \cong T_{1} k_{1} \times D^{2}$ tomemos o nó $k(r, s)$, onde $T_{1}$ é a trivialização distinguida de vizinhança tubular do nó $k_{1}$. Este nó está contido no toro $k_{1} \times S^{1} \subset D^{3}$. Denotemos este nó por $k_{2}$.

Então verificamos que $S\left(k_{2}\right)$ é obtido através de $g: S(k(p, q)) \rightarrow B_{0, r}$ associada a:

$$
\begin{aligned}
& g \pi: \pi_{1}(S(k(p, q))) \longrightarrow \pi_{1}\left(B_{0, r}\right), \\
& g \pi([k(p, q)])=W_{r, s}, \quad g \pi\left(\left[S^{1}\right]\right)=i d .
\end{aligned}
$$

Sabemos que [29] o polinômio de Alexander do nó no toro do tipo $(p, q)$ é 


$$
\Delta(t)=\frac{(1-t)\left(1-t^{p q}\right)}{\left(1-t^{p}\right)\left(1-t^{q}\right)}
$$

Assim utilizando a relação de Seifert do Teorema II da seção 4 obtemos o polinômio de Alexander de $k_{2}$ :

$$
\Delta_{k_{2}}(t)=\frac{(1-t)\left(1-t^{r s}\right)}{\left(1-t^{r}\right)\left(1-t^{s}\right)} \cdot \frac{\left(1-t^{r}\right)\left(1-t^{r p q}\right)}{\left(1-t^{r p}\right)\left(1-t^{r q}\right)} .
$$

Teremos os seguintes módulos de Alexander:

$$
\begin{gathered}
H_{1}\left(\tilde{X}_{M}\right) \cong \frac{\Lambda}{\Delta_{k_{2}}(t)}, \\
H_{2}\left(\tilde{X}_{M}\right) \cong \Lambda^{2} \oplus \frac{\Lambda}{\Delta_{k_{2}}(t)} .
\end{gathered}
$$




\section{Referências}

[1] Artin E., Theory of Braids, Annals of Math. Vol 48, 1(1947), 101-126.

[2] Artin E., Braids and Permutations, Annals of Math. Vol 48, 3(1947), 643649.

[3] Atiyah M.' F. \& MacDonald I. G., Introduction to Comutative Algebra, Addison-Wesley Publishing Company, Londres, 1969.

[4] Balley J. L., Alexander Invariants of Links, PhD Thesis, Univ. of British Columbia, June, 1977.

[5] Birman J. S., Braids, Links and Mapping Class Groups, Annals of Math. Studies 82, Princeton Univ. Press, New Jersey, 1975.

[6] Bohnenblust F., The Algebraical Braid Group, Annals of Math. Vol 48, 1(1947), 127-136.

[7] Brown K. S. , Cohomology of groups, Springer-Verlag, New York, 1982.

[8] Cartan H. \& Eilenberg S., Homological Algebra, Princeton Univ. Press, New Jersey, 1956.

[9] Chow W.L., On the Algebraical Braid Group, Annals of Math. Vol 49, 3(1948), 654-658

[10] Cornea Otav, The Genus and The Fundamental Group of High Dimensional Manifold, Stud. Cerc. Mat. Tomo 41, 3 (1989) Bucareste, 169-178.

[11] Gordon C. McA., Homology of groups Surfaces in the 4-Space, Math. Proc. Camb. Phil. Soc. (1981), 89-113.

[12] Hopf H., Relations between the Fundamental Group and the Second Betti Group, Lectures in Topology - The University of Michigan Conference of 1940, 315-316.

[13] Hosokawa F., Maeda T.,\& Suzuki S., Numerical Invariants of Surfaces in 4-Space, Kobe Math. Seminar Notes Vol 7, (1979).

[14] Iwase Z., Dehn-Surgery along a torus $T^{2}$-knot, Pacific J. Math. Vol. 133, 2 (1988), 280-299. 
[15] Kanenobu T., Higher Dimentional Cable Knots and Their Finite Cyclic Covering Spaces, Topology and its Applications, 19 (1985), 123-127.

[16] Kawauchi A. \& Hosokawa F., Proposals for Unknotted Surfaces in FourSpaces, Osaka J. Math., 16 (1979), 233-248.

[17] Knigth J. T., Comutative Algebra, Cambridge Univ. Press, Oxford, 1971.

[18] Levine J., Knot Modules I, Trans. of A.M.S. Vol 229, 1977, 1-50.

[19] Livingston C. \& Melvin P., Abelian Invariants of Satellite Knots, Lecture Notes in Math. 1167, Springer, Berlin, 1985, 217-227.

[20] MacLane S., Homology, Springer-Verlag, Berlin, 1967.

[21] McCleary J., User's Guide to Spectral Sequences, Math. Lecture Series 12, Publish or Perish,Inc. , Wilmington, Delaware (USA), 1985.

[22] Milnor .J. W. \& Stasheff J.D., Characteristic Classes, Annals of Math. Studies 76, Princeton Univ. Press, New Jersey, 1974.

[23] Milnor J. W., Infinite Ciclic Coverings, Conference on the Topology of Manifolds (Michigan State Univ., 1967), Prindle, Weber and Schimidt, Boston, Mass., 1968, 115-133.

[24] Milnor J. W., A Duality Theorem for Reidemeister Torsion, Annals of Math. Vol 76, 1 (1962), 137-147.

[25] Montesinos J. M., On twins in the four-sphere, Quart. J. Math. Oxford (2),34 (1983),171-199.

[26] Neto O. M., Total Linking Number Modules, Trans. of A.M.S. Vol 307, 2 (1988), 503-533.

[27] Neto O.M., Seifert Surfaces for Codimension Two Links of Manifolds in Spheres, Pré-print.

[28] Randall D. \& DaCcach J., Sequências Espectrais, Espaços de EilembergMacLane, Torres de Postnikov, Notas Didáticas do ICMSC-USP, São Carlos, 1988.

[29] Rolfsen D., Knots and Links, Publish or Perish,Inc. , Berkeley, 1976.

[30] Seifert H., On the Homology Invariants of Knots, Quart. J. Math. Oxford (2), 1 (1950), 23-32. 
[31] Sekine M., Kawauchi's Second Duality and Knotted Surfaces in 4-Sphere, Hiroshima Math. J., 19 (1989), 641-651.

[32] Simon T., Wirtinger Aproximations and The Knot Group of $F^{n}$ in $S^{n+2}$, Pacific Journal of Maths. Vol 90, 1 (1980), 177-189.

[33] Shinohara Y., Higher Dimensional Knots in Tubes, Trans. of A.M.S. Vol 161, (1971), 35-49.

[34] Shinohara Y., On the Signature of Knots and Links, Trans. of A.M.S. Vol 156, (1971), 273-285.

[35] SPAnier E. W., Algebraic Topology, MacGraw-Hill Book Company, New York, 1966. 